\title{
Quantum melting of magnetic long-range order near orbital degeneracy: Classical phases and Gaussian fluctuations
}

\author{
Andrzej M. Oleś \\ Institute of Physics, Jagellonian University, Reymonta 4, PL-30059 Kraków, Poland \\ and Max-Planck-Institut für Festkörperforschung, Heisenbergstrasse 1, D-70569 Stuttgart, Federal Republic of Germany \\ Louis Felix Feiner \\ Institute for Theoretical Physics, Utrecht University, Princetonplein 5, NL-3584 CC Utrecht, The Netherlands \\ and Philips Research Laboratories, Prof. Holstlaan 4, NL-5656 AA Eindhoven, The Netherlands \\ Jan Zaanen \\ Lorentz Institute for Theoretical Physics, Leiden University, P.O.B. 9506, NL-2300 RA Leiden, The Netherlands
}

(Received 25 March 1999)

\begin{abstract}
We address the role played by orbital degeneracy in strongly correlated transition-metal compounds. Specifically, we study the effective spin-orbital model derived for the $d^{9}$ ions in a three-dimensional perovskite lattice, as in $\mathrm{KCuF}_{3}$, where at each site the doubly degenerate $e_{g}$ orbitals contain a single hole. The model describes the superexchange interactions that depend on the pattern of orbitals occupied and shows a nontrivial coupling between spin and orbital variables at nearest-neighbor sites. We present the ground-state properties of this model, depending on the splitting between the $e_{g}$ orbitals $E_{z}$, and the Hund's rule coupling in the excited $d^{8}$ states, $J_{H}$. The classical phase diagram consists of six magnetic phases which all have different orbital ordering: two antiferromagnetic (AF) phases with $\mathrm{G}-\mathrm{AF}$ order and either $x^{2}-y^{2}$ or $3 z^{2}-r^{2}$ orbitals occupied, two phases with mixed orbital (MO) patterns and A-AF order, and two other MO phases with either C-AF or G-AF order. All of them become degenerate at the multicritical point $M \equiv\left(E_{z}, J_{H}\right)=(0,0)$. Using a generalization of linear spin-wave theory we study both the transverse excitations which are spin waves and spin-andorbital waves, as well as the longitudinal (orbital) excitations. The transverse modes couple to each other, providing a possibility of measuring the new spin-and-orbital excitations in inelastic neutron spectroscopy. As the latter excitation turns into a soft mode near the $M$ point, quantum corrections to the long-range-order parameter are drastically increased near the orbital degeneracy, and classical order is suppressed in a crossover regime between the $\mathrm{G}-\mathrm{AF}$ and $\mathrm{A}-\mathrm{AF}$ phases in the $\left(E_{z}, J_{H}\right)$ plane. This behavior is reminiscent of that found in frustrated spin models, and we conclude that orbital degeneracy provides a different and physically realizable mechanism which stabilizes a spin liquid ground state due to inherent frustration of magnetic interactions. We also point out that such a disordered magnetic phase is likely to be realized at low $J_{H}$ and low electronphonon coupling, as in $\mathrm{LiNiO}_{2}$.
\end{abstract}

\section{NOVEL MECHANISM OF FRUSTRATION NEAR ORBITAL DEGENERACY}

Quite generally, strongly correlated electron systems involve orbitally degenerate states, ${ }^{1}$ such as $3 d(4 d)$ states in transition metal compounds, and $4 f(5 f)$ states in rare-earth compounds. Yet, the orbital degrees of freedom are ignored in most situations and the common approach is to consider a single correlated orbital per atom which leads to spin degeneracy alone. Indeed, most of the current studies of strongly correlated electrons deal with models of nondegenerate orbitals. The problems discussed recently include mechanisms of ferromagnetism in the Hubbard model, ${ }^{2}$ hole propagation and quasiparticles in the $t$ - $J$ model, ${ }^{3}$ and magnetic states of the Kondo lattice. ${ }^{4}$ Of course, in many actually existing compounds the orbital degeneracy is removed by the crystal field, and a single-orbital approach is valid per se. Also, from a fundamental point of view it is often possible to argue that orbital degeneracy is qualitatively irrelevant, and that a single-orbital approach can capture the generic mechanisms operative in the presence of strong correlations.
However, neither of these arguments applies for a class of insulating strongly correlated transition-metal compounds, where the crystal field leaves the $3 d$ orbitals explicitly degenerate and thus the type of occupied orbitals is not known a priori, while the magnetic interaction between the spins of neighboring transition-metal ions depends on which orbitals are occupied. In this particular class of Mott-Hubbard insulators (MHI) the orbital degrees of freedom acquire a separate existence in much the same way as the spins do. Thereby, the degeneracy of $t_{2 g}$ orbitals is of less importance, as the magnetic superexchange and the coupling to the lattice are rather weak. A more interesting situation occurs when $e_{g}$ orbitals are partly occupied, which results in stronger magnetic interactions, and strong Jahn-Teller (JT) effect. Typical examples of such ions are: $\mathrm{Cu}^{2+}\left(d^{9}\right.$ configuration, one hole in $e_{g}$-orbitals), low-spin $\mathrm{Ni}^{3+}$ ( $d^{7}$ configuration, one electron in $e_{g}$ orbitals), as well as $\mathrm{Mn}^{3+}$ and $\mathrm{Cr}^{2+}$ ions (high-spin $d^{4}$ configuration, one $e_{g}$ electron). The simplest model, relevant for $d^{9}$ transition-metal ions, which is also the subject of the present paper, was introduced by Kugel and Khomskii more than two decades ago, ${ }^{5}$ but its mean-field (MF) phase dia- 
gram was analyzed only recently. ${ }^{6}$ It describes magnetic superexchange interactions between spins $S=1 / 2$, and the accompanying orbital superexchange interactions.

One might argue that the (classical) orbital degeneracy is not easy to realize in such systems, as the electron-phonon coupling will lead to the conventional collective JT instability. In fact, it can be shown that the JT instability is enhanced by the orbital pattern once this has been established as the result of effective interactions: $:^{5,7,8}$ the lattice has to react to the symmetry lowering in the orbital sector, which can only increase the stability of a given magnetic state. So the lattice follows rather than induces the orbital order, and therefore, as was pointed out in the early work by Kugel and Khomskii, ${ }^{5,9}$ in the orbitally degenerate MHI one has to consider in first instance the purely electronic problem. This is supported by the results of recent band-structure calculations using the local-density approximation (LDA) with the electron interactions treated in Hartree-Fock approximation, the so-called LDA $+U$ method, which permits both orbitals and spins to polarize while keeping the accurate treatment of the electron-lattice coupling of LDA intact. These calculations reproduce the observed orbital ordering in $\mathrm{KCuF}_{3}$ (Ref. 10) and in $\mathrm{LaMnO}_{3}$, ${ }^{11}$ even when the lattice distortions are suppressed, while allowing the lattice to relax only yields an energy gain which is minute in comparison with the energies involved in the orbital ordering.

Effects of orbital degeneracy are expected as soon as crystal-field splittings become small. Such situations are frequently encountered in rare-earth systems, where they lead to the so-called singlet-triplet models discussed in the seventies, ${ }^{12}$ while in the $3 d$ oxides only a small number of so-called Kugel-Khomskii (KK) systems ${ }^{9}$ have been recognized that actually exhibit orbital effects. ${ }^{7}$ As pointed out by Kugel and Khomskii, ${ }^{5}$ in such situations the superexchange interactions have a more complex form than in spin-only models and one expects that also in some other MottHubbard (or charge-transfer) insulators new magnetic phases might arise due to the competition of various magnetic and orbital interactions. Some examples of such a competition of magnetic interactions are encountered in the heavy fermion systems, ${ }^{4,13}$ and in the manganites where the phase diagrams show a particular frustration of magnetic interactions. ${ }^{14-17}$

Even more interesting behavior is expected for the doped systems, as the competition between the magnetic, orbital, and kinetic energy is then described by $t-J$ Hamiltonians of a novel type, which exhibit qualitatively different excitation spectra due to the underlying orbital degeneracy. ${ }^{18} \mathrm{~A}$ few examples of such models have already been discussed in the literature, such as the triplet $t-J$ model, ${ }^{19}$ the low-spin defects in a $S=1$ background, ${ }^{20}$ or a $t$ - $J$-like model for the manganites. ${ }^{21}$ Whether such models are realistic enough is not yet clear, as, for example, in the manganites there are experimental $^{22}$ and theoretical ${ }^{23}$ indications that the doubleexchange model which includes only the spin degrees of freedom is insufficient to understand the transport properties under doping. Recent work ${ }^{16,17,24,25}$ strongly suggests that an extension of the $t-J$ and double-exchange models which include fully the orbital physics should be studied instead.

In this paper we shall consider only the insulating situation, where one can integrate out the $d-d$ excitations and derive an effective low-energy Hamiltonian. This approach is justified by the large on-site Coulomb interaction $U$, being the largest energy scale in MHI. A low-energy Hilbert space splits off, spanned by spin and orbital configuration space, with superexchangelike couplings between both spin and orbital local degrees of freedom. The orbital sector carries a discrete symmetry and the net outcome is that the clocklike orbital degrees of freedom get coupled into the SU(2) spin problem. The resulting low-energy Hamiltonian is called a spin-orbital model. Here we focus on the simplest situation with two nearly degenerate partially filled $e_{g}$ orbitals, and completely filled $t_{2 g}$ orbitals, as encountered in $\mathrm{KCuF}_{3}$ and related systems. ${ }^{9}$ These are JT-distorted cubic crystals, threedimensional (3D) analogs of the cuprate superconductors. ${ }^{26}$ In the high- $T_{c}$ cuprates, orbital degeneracy would occur if the $\mathrm{Cu}-\mathrm{O}$ bonds which involve apical oxygens were squeezed such as to recover the cubic symmetry of the perovskite lattice. Of course, such a degeneracy of $e_{g}$ orbitals is far from being realized in the actual high- $T_{c}$ materials, and in their parent compounds. ${ }^{27,28}$

If only one correlated orbital is present, the system may be described by the effective single-band Hubbard model (typically with more extended hopping), as in the cuprate superconductors. ${ }^{29}$ In this simplest case the effective model at half filling is the Heisenberg model with antiferromagnetic (AF) superexchange. This changes when more than one $3 d$ orbital is partly occupied. For example, we show in Sec. II that virtual excitations involving $d^{8}$ local triplet states become possible in the case of degenerate $e_{g}$ orbitals, and this leads to additional ferromagnetic (FM) interactions. The origin of these interactions was first discussed by Kugel and Khomskii ${ }^{5}$ and by Cyrot and Lyon-Caen ${ }^{30}$ who pointed out that the strongest superexchange constant results from the excitation to the lowest energy triplet state in the degenerate Hubbard model. The superexchange interaction in doubly degenerate band with arbitrary filling was somewhat later analyzed by Spałek and Chao, who derived a generalized $t-J$ model for $e_{g}$ electrons. $^{31}$

The model proposed by Kugel and Khomskii explains qualitatively the observed magnetic ordering in $\mathrm{KCuF}_{3}$ as being due to an orbital ordering which gives planes of perpendicularly oriented orbitals, and the magnetic coupling becomes then FM according to the Goodenough-Kanamori rules. ${ }^{32}$ As mentioned above, such a state was indeed found in the band structure calculations of Liechtenstein, Anisimov, and Zaanen ${ }^{10}$ using the LDA $+U$ method. An analogous orbital order is responsible for ferromagnetism in the planar FM insulator $\mathrm{K}_{2} \mathrm{CuF}_{4}{ }^{33}$ In the colossal magnetoresistance parent compound $\mathrm{LaMnO}_{3}$, where the $e_{g}$ orbitals contain one electron instead of one hole, a similar orbital ordering occurs, ${ }^{7,15}$ although the situation there is more complex due to the presence of $t_{2 g}$ spins, so that the resulting superexchange is not between spins $S=1 / 2$ but between total spins $S=2 .{ }^{17}$ Another example of degenerate orbitals is found in $\mathrm{V}_{2} \mathrm{O}_{3}$, with the orbital ordering studied by Castellani, Natoli, and Ranninger in a series of papers. ${ }^{34}$ In fact, their prediction that the transition into the AF insulator is accompanied by the onset of orbital ordering was experimentally verified only recently. ${ }^{35}$ However, this case is still open, as recent electronic structure calculations suggest that doubly degenerate orbitals are occupied by two electrons in the high-spin state and the orbital degree of freedom plays no role. ${ }^{36}$ 
In any of the above situations the orbital ordering breaks the translational symmetry and represents an analog of spin antiferromagnetism in orbital space. So, classically orbital ordering is expected to occur quite generally whenever one encounters $e_{g}$ orbitals containing either one hole or one electron, with important consequences for the magnetism. This immediately raises a number of questions about what happens in the quantum regime. Will orbital long-range order (LRO) be robust or will it give way to an orbital liquid, as proposed by Ishihara, Yamanaka, and Nagaosa? ${ }^{37}$ In either case, what are the consequences of the enlarged phase space and the associated additional channels for quantum fluctuations for the magnetism: can magnetic LRO survive or will it be replaced by a spin liquid?

Quantum disordered phases are of great current interest. Spin disorder is well known to occur in one-dimensional (1D) and quasi-1D quantum spin systems, and the best example is the 1D Heisenberg model, where the famous exact solution found by Bethe many years ago ${ }^{38}$ showed that the quantum fluctuations prevent true AF LRO, giving instead a slow decay of spin correlations. A similar situation is encountered in spin ladders with an even number of legs, which have a spin gap and purely short-range magnetic order. ${ }^{39,40}$ This is one of the realizations of a spin-liquid ground state due to purely short-range spin correlations. In the limit of a two-dimensional (2D) Heisenberg model the spin disorder is replaced by a ground state with AF LRO.

It is well known that frustrated magnetic interactions may lead to spin disordered states in two dimensions. However, in order to achieve this, i.e., to prevent 2D macroscopic spin systems from behaving classically and to make quantum mechanics take over instead, the frustration of the interactions must be sufficiently severe. This shows that global $S U(2)$ by itself is not symmetric enough to defeat classical order in $D>1$ and one has to change the magnetic interactions in such a way that they lead to sufficiently strong quantum fluctuations. So far, this strategy has been shown to lead to spin disorder in (quasi-)2D systems in three different situations: (i) Frustrating a 2D square lattice by adding longerrange AF interactions, as in $J_{1}-J_{2}$ and $J_{1}-J_{2}-J_{3}$ models, gives a high degeneracy of the classical sector, and a disordered state is found for particular values of the magnetic interactions. $^{41,42}$ This mechanism involves fine tuning of parameters and therefore such systems are hard to realize in nature. (ii) In the bilayer Heisenberg model two planes are coupled by interlayer AF superexchange $J_{\perp}$ which generates zero-dimensional fluctuations. This leads to a crossover to the disordered ground state of an incompressible spin liquid above a certain critical value of $J_{\perp} \cdot{ }^{43,44}$ Also this mechanism is hard to realize experimentally. (iii) In contrast, a spin disordered state can be obtained in nature by reducing the number of magnetic bonds in a $2 \mathrm{D}$ square lattice. The model of $\mathrm{CaV}_{4} \mathrm{O}_{9}$ studied by Taniguchi et al. ${ }^{45}$ is a $1 / 5$ depleted square lattice, which gives a plaquette resonating valence bond (PRVB) ground state for realistic interactions, and a spin gap which agrees with experimental observations. ${ }^{46} \mathrm{~A}$ common feature of these systems is a crossover between different magnetic ground states, either between two different patterns of LRO, as in case (i), or simply between the ordered and disordered states, which results in all three situations in a tendency towards the formation of spin singlets on the bonds with the strongest AF superexchange. One may further note that in these spin-only models very specific patterns of magnetic interactions are required already in two dimensions to prevent the system to order classically, while up to now it has proven impossible to realize a spin liquid in three dimensions.

In the present paper we address two fundamental questions for the Heisenberg antiferromagnet (HAF) extended to include the orbital degrees of freedom in orbitally degenerate MHI: (i) Which classical states with magnetic LRO do exist in the neighborhood of orbital degeneracy? (ii) Are those forms of classical order always stable against quantum fluctuations? We will show that the orbitally degenerate MHI represent a class of systems in which spin disorder occurs due to frustration of spin and orbital superexchange couplings. This frustration mechanism is different from that operative in pure spin systems, and suppresses the magnetic LRO in the ground state even in three dimensions.

As explained above, the low-energy behavior of such systems is described by a spin-orbital model. We will show that within the framework of such a spin-orbital model the occurrence of spin disorder may be regarded as resulting from a competition between various classical ordered phases, each one with a simultaneous symmetry breaking in spin and orbital space. As we show below (see Sec. III), there are two types of classical AF phases without an orbital order, i.e., when all the orbitals are the same: a 2D phase with $x^{2}-y^{2}$ orbitals occupied by spins, the so-called AFxx phase, and an anisotropic 3D phase with $3 z^{2}-r^{2}$ orbitals occupied by spins, the so-called AFzz phase, next to a few phases with mixed orbitals (MO's) which stagger and lead to MO phases, typically with FM interactions in at least one spatial direction. Thus the qualitatively new aspect is that the magnetic interactions follow the orbital pattern, and thus these systems tend to "self-tune" to (critical) points of high classical degeneneracy. We show explicitly that in the vicinity of such a multicritical point classical order is highly unstable with respect to quantum fluctuations. As a result, a qualitatively different quantum spin liquid with strong orbital correlations is expected. We believe that a 3D state of this type is realized in $\mathrm{LiNiO}_{2}$.

The paper is organized as follows. The spin-orbital model for $d^{9}$ transition-metal ions, such as $\mathrm{Cu}^{2+}$ ions in $\mathrm{KCuF}_{3}$, is derived in Sec. II using the correct multiplet structure of $\mathrm{Cu}^{3+}$ excited configurations. We solve this model first in the MF approximation and present the resulting classical phases and the accompanying orbital orderings in Sec. III. The elementary excitations obtained within an extension of the linear spin-wave (LSW) theory are presented in Sec. IV, where we demonstrate that two transverse modes are strongly coupled to each other. This leads to soft modes next to the classical transition lines, and to the collapse of LRO due to diverging quantum corrections, as shown in Sec. V. We summarize the results and present our conclusions in Sec. VI.

\section{THE SPIN-ORBITAL MODEL}

Our aim is to construct the effective low-energy Hamiltonian for a 3D perovskitelike lattice. The original chargetransfer multiband model, as considered for instance for the cuprates, includes the hybridization elements between the $3 d$ 
orbitals of transition-metal ions and the $2 p$ orbitals of oxygen ions. ${ }^{29}$ If the Coulomb elements at the $3 d$ orbitals and the charge-transfer energy between the $3 d$ and $2 p$ orbitals are large, this model can be transformed into an effective spin-fermion model. For example, this transformation performed for the three-band model gives an effective Hamiltonian with localized spins at the $\mathrm{Cu}$ sites which interact by superexchange interactions, while the doped carriers interact with them by a Kondo-like coupling. ${ }^{47}$ In the limit of undoped compounds, one is thus left with a model which describes interacting transition-metal ions.

The simplest form of (superexchange) interaction, namely a purely spin model, is obtained for the case of nondegenerate $d$ orbitals, whereas orbital degeneracy gives a spin-orbital model acting in a larger Hilbert space defined by both spin and orbital degrees of freedom at each transition-metal site. Having in mind the strongly correlated late transition-metal oxides, we consider specifically the case of one hole per unit cell in the $3 d^{9}$ configuration, characterized in the absence of JT distortion by two degenerate $e_{g}$ orbitals: $x^{2}-y^{2} \sim|x\rangle$ and $\left(3 z^{2}-r^{2}\right) / \sqrt{3} \sim|z\rangle$. The derivation is, however, more general and applies as well to the low-spin $d^{7}$ configuration; in the case of the early transition-metal oxides the $d^{1}$ case would involve the $t_{2 g}$ orbitals instead.

The holes in the undoped compound which corresponds to the $d^{9}$ configuration of transition-metal ions, as in $\mathrm{La}_{2} \mathrm{CuO}_{4}$ or $\mathrm{KCuF}_{3}$, are fairly localized. ${ }^{48}$ Hence we take as a starting point the following Hamiltonian which describes $d$ holes on transition-metal ions:

$$
H_{e_{g}}=H_{k i n}+H_{i n t}+H_{z},
$$

and consider the kinetic energy $H_{k i n}$ and the electronelectron interactions $H_{\text {int }}$ within the subspace of the $e_{g}$ orbitals (the $t_{2 g}$ orbitals are filled by electrons, do not couple to $e_{g}$ orbitals due to the hoppings via oxygens, and hence can be neglected). The last term $H_{z}$ describes the crystal-field splitting of the $e_{g}$ orbitals.

Due to the shape of the two $e_{g}$ orbitals $|x\rangle$ and $|z\rangle$, their $d-p$ hybridization in the three cubic directions is unequal, and is different between them, so that the effective hopping elements are direction dependent and different for $|x\rangle$ and $|z\rangle$. The only nonvanishing hopping in the $c$ direction connects two $|z\rangle$ orbitals, while the elements in the $(a, b)$ planes fulfill the Slater-Koster relations, ${ }^{49}$ as presented before by two of us. ${ }^{18}$ Taking the hopping $t$ along the $c$ axis as a unit, the kinetic energy is given by

$$
\begin{aligned}
H_{k i n}= & \frac{t}{4} \sum_{\langle i j\rangle \|}\left[3 d_{i x \sigma}^{\dagger} d_{i x \sigma}+(-1)^{\vec{\delta} \cdot \vec{y}} \sqrt{3}\left(d_{i z \sigma}^{\dagger} d_{i x \sigma}+\text { H.c. }\right)\right. \\
& \left.+d_{i z \sigma}^{\dagger} d_{i z \sigma}\right]+t \sqrt{\beta} \sum_{\langle i j\rangle \perp} d_{i z \sigma}^{\dagger} d_{i z \sigma}
\end{aligned}
$$

where $\langle i j\rangle \|$ and $\langle i j\rangle \perp$ stand for the bonds between nearest neighbors within the $(a, b)$ planes, and along the $c$ axis, respectively, and $\beta=1$ in a cubic system. The $x-z$ hopping in the $(a, b)$ planes depends on the phases of the $x^{2}-y^{2}$ orbitals along $a$ and $b$ axis, respectively, included in the factors $(-1)^{\vec{\delta} \cdot \vec{y}}$ in Eq. (2.2).

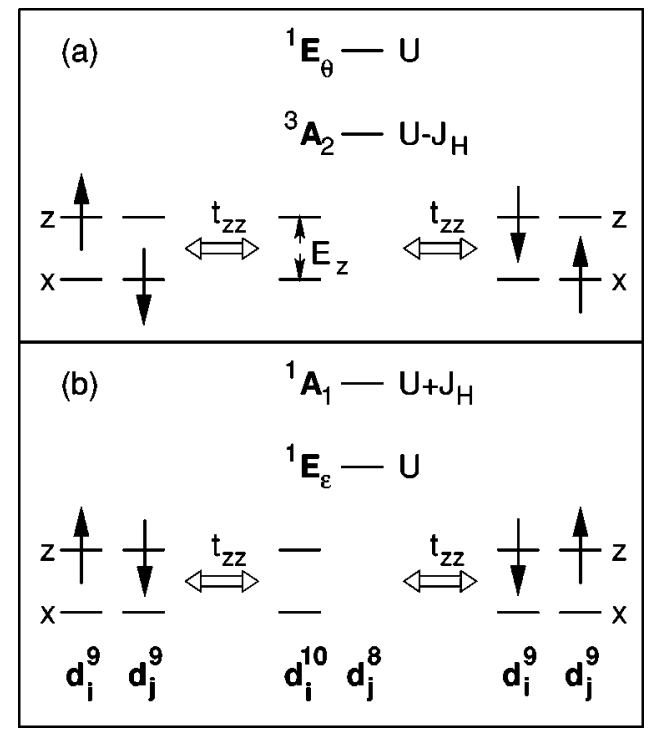

FIG. 1. Virtual transitions $d_{i}^{9} d_{j}^{9} \rightarrow d_{i}^{10} d_{j}^{8}$ which lead to a spin-flip and generate effective interactions for a bond $\langle i j\rangle \| c$ axis, with the excitation energies at $E_{z}=0$. For two holes in different orbitals (a), either the triplet ${ }^{3} A_{2}$ or the interorbital singlet ${ }^{1} E_{\theta}$ occurs as an intermediate $d^{8}$ configuration, while if both holes are in $|z\rangle$ orbitals (b), two other singlets, ${ }^{1} E_{\epsilon}$ and ${ }^{1} A_{1}$, with double occupancy of $|z\rangle$ orbital, contribute. The latter processes are possible either by hole hopping from $i$ to $j$ or from $j$ to $i$.

The electron-electron interactions are described by the onsite terms

$$
\begin{aligned}
H_{i n t}= & \left(U+\frac{1}{2} J_{H}\right) \sum_{i \alpha} n_{i \alpha \uparrow} n_{i \alpha \downarrow}+\left(U-J_{H}\right) \sum_{i \sigma} n_{i x \sigma} n_{i z \sigma} \\
& +\left(U-\frac{1}{2} J_{H}\right) \sum_{i \sigma} n_{i x \sigma} n_{i z \bar{\sigma}}-\frac{1}{2} J_{H} \sum_{i \sigma} d_{i x \sigma}^{\dagger} d_{i x \bar{\sigma}} d_{i z \bar{\sigma}}^{\dagger} d_{i z \sigma} \\
& +\frac{1}{2} J_{H} \sum_{i}\left(d_{i x \uparrow}^{\dagger} d_{i x \downarrow}^{\dagger} d_{i z \downarrow} d_{i z \uparrow}+d_{i z \uparrow}^{\dagger} d_{i z \downarrow}^{\dagger} d_{i x \downarrow} d_{i x \uparrow}\right),
\end{aligned}
$$

with $U$ and $J_{H}$ standing for the Coulomb and Hund's rule exchange interaction, ${ }^{50}$ respectively, and $\alpha=x, z$. For convenience, we used the simplified notation $\bar{\sigma}=-\sigma$. This Hamiltonian describes correctly the multiplet structure of $d^{8}$ (and $d^{2}$ ) ions, ${ }^{51}$ and is rotationally invariant in the orbital space. ${ }^{52}$ The wave functions have been assumed to be real which gives the same element $J_{H} / 2$ for the exchange interaction and for the "pair hopping"' term between the $e_{g}$ orbitals, $|x\rangle$ and $|z\rangle$.

In fact, we adopted here the most natural units for the elements of the Coulomb interaction, with the energy of the central $\left|{ }^{1} E\right\rangle$ doublet being equal to $U$. By definition this energy does not depend on the Hund's exchange element $J_{H}$, as we show below, and is thus the measure of the average excitation energy in the $d_{i}^{9} d_{j}^{9} \rightarrow d_{i}^{10} d_{j}^{8}$ transition. The interaction element $J_{H}$ stands for the singlet-triplet splitting in the $d^{8}$ spectrum (Fig. 1) and is just twice as big as the exchange element $K_{x z}$ used usually in quantum chemistry. ${ }^{28}$ The typical energies for the Coulomb and exchange elements can be found using constrained-occupation local-density functional theory. ${ }^{53}$ Unfortunately, such calculations have 
been performed only for a few compounds so far. For $\mathrm{La}_{2} \mathrm{CuO}_{4}$, a parent compound of superconducting cuprates, one finds $U=7.77 \mathrm{eV}$ and $J_{H}=2.38 \mathrm{eV} ;{ }^{28}$ other estimations of $U$ based on the experimental data report values $6<U$ $<8 \mathrm{eV}$ for cuprates and nickelates. ${ }^{54}$ This results in the ratio $J_{H} / U \simeq 0.3$ which we take as a representative value for the strongly correlated late transition-metal oxides. The values of intersite hopping $t$, being an effective parameter, are more difficult to estimate. As a representative value for $\mathrm{La}_{2} \mathrm{CuO}_{4}$ one might take $t \approx 0.65 \mathrm{eV}$, which results in the superexchange interaction between the $|x\rangle$ orbitals in $(a, b)$ planes, $J_{(a, b)}=(9 / 4) t^{2} / U \simeq 0.13 \mathrm{eV},{ }^{55}$ in good agreement with the experimental value. ${ }^{56}$ Similar values of the effective $t$ are expected also in the other transition-metal oxides, and thus we can safely assume that at the filling of one hole per ion the ionic Hamiltonian (2.1) describes an insulating state, and that the effective magnetic interactions can be derived in the strongly correlated regime of $t \ll U$.

The last term in Eq. (2.1) stands for the crystal field which lifts the degeneracy of the two $e_{g}$ orbitals and breaks the symmetry in the orbital space,

$$
H_{z}=\sum_{i \sigma}\left(\varepsilon_{x} n_{i x \sigma}+\varepsilon_{z} n_{i z \sigma}\right)
$$

if $\varepsilon_{x} \neq \varepsilon_{z}$. It acts as a magnetic field in the orbital space, and together with the parameter $\beta$ in $H_{\text {kin }}$ (2.2) quantifies the deviation in the electronic structure from the ideal cubic local point group.

In the atomic limit, i.e., at $t=0$ and $E_{z}=0$, one has orbital degeneracy next to spin degeneracy. This gives four basis states per site, as each hole may occupy either orbital, $|x\rangle$ or $|z\rangle$, and either spin state, $\sigma=\uparrow$ or $\sigma=\downarrow$. The system of $N d^{9}$ ions has thus a large degeneracy $4^{N}$, which is, however, removed by the effective interactions between each pair of nearest-neighbor ions $\{i, j\}$ which originate from virtual transitions to the excited states, $d_{i}^{9} d_{j}^{9} \rightleftharpoons d_{i}^{10} d_{j}^{8}$, due to hole hopping. Hence we derive the effective spin-orbital model following Kugel and Khomskii, ${ }^{5}$ starting from the Hamiltonian in the atomic limit, $H_{a t}=H_{i n t}+H_{z}$, and treating $H_{k i n}$ as a perturbation. However, in the present study we include the full multiplet structure of the excited states within the $d^{8}$ configuration which gives corrections of the order of $J_{H}$ compared with the earlier results of Refs. 5 and 9.

Knowing the multiplet structure of the $d^{8}$ intermediate states, the derivation of the effective Hamiltonian can be done in various ways. The most straightforward but lengthy procedure is a generalization of the canonical transformation method used before for the Hubbard ${ }^{57}$ and the three-band ${ }^{47}$ model. A significantly shorter derivation is possible, however, using the cubic symmetry and starting with the interactions along the $c$ axis. Here the derivation simplifies tremendously as one finds only effective interactions which result from the hopping of holes between the directional $|z\rangle$ orbitals, as shown in Fig. 1. Next the interactions in the remaining directions can be generated by the appropriate rotations to the other cubic axes $a$ and $b$, and applying the symmetry rules for the hopping elements between the $e_{g}$ orbitals. ${ }^{49}$ The derivation of the spin-orbital model is given in more detail in Appendix A.
Depending on whether the initial state is $|z\rangle_{i}|x\rangle_{j}$ or $|z\rangle_{i}|z\rangle_{j}$, the intermediate $d_{i}^{10} d_{j}^{8}$ configuration resulting from the hole-hop $|z\rangle_{i} \rightarrow|z\rangle_{j}$, involves on the $d^{8}$ site either the interorbital states, the triplet ${ }^{3} A_{2}$ and the singlet ${ }^{1} E_{\theta}$, or the two singlets built from the states with doubly occupied orbitals, ${ }^{1} E_{\varepsilon}$ and ${ }^{1} A_{1}$. Of course, the spins have to be opposite in the latter case, while in the former case also parallel spin configurations contribute in the triplet channel. Apart from a constant term, this atomic problem is equivalent to that of the $d^{2}$ configuration, and thus one might consider instead the spectrum of $d^{2}$ ions. The eigenstates within the $e_{g}$ subspace are: (i) triplet $\left|{ }^{3} A_{2}\right\rangle$, (ii) interorbital singlet $\left|{ }^{1} E_{\epsilon}\right\rangle^{g}$, and (iii) bonding and antibonding singlets, $\left|{ }^{1} E_{\theta}\right\rangle$ and $\left|{ }^{1} A_{1}\right\rangle$, with double occupancies of both orbitals, where bonding/ antibonding refers to pair hopping term $\propto J_{H}$ between $|x\rangle$ and $|z\rangle$ orbital. The energies of the states $\left|{ }^{3} A_{2}\right\rangle$ and $\left|{ }^{1} E_{\epsilon}\right\rangle$ are straighforwardly obtained using $\vec{S}_{i x} \cdot \vec{S}_{i z}=+1 / 4$ and $\vec{S}_{i x} \cdot \vec{S}_{i z}$ $=-3 / 4$, for $S=1$ and $S=0$ states, respectively. The remaining two singlet energies are found by diagonalizing a $2 \times 2$ problem in the subspace of doubly occupied states. Hence the resulting spectrum is ${ }^{58}$

$$
\begin{gathered}
E\left({ }^{3} A_{2}\right)=U-J_{H}, \\
E\left({ }^{1} E_{\epsilon}\right)=U, \\
E\left({ }^{1} E_{\theta}\right)=U+\frac{1}{2} J_{H}-\frac{1}{2} J_{H}\left[1+\left(E_{z} / J_{H}\right)^{2}\right]^{1 / 2}, \\
E\left({ }^{1} A_{1}\right)=U+\frac{1}{2} J_{H}+\frac{1}{2} J_{H}\left[1+\left(E_{z} / J_{H}\right)^{2}\right]^{1 / 2},
\end{gathered}
$$

where $E_{z}=\varepsilon_{x}-\varepsilon_{z}$. At $E_{z}=0$ it consists of equidistant states, with a distance of $J_{H}$ between the triplet $\left|{ }^{3} A_{2}\right\rangle$ and the degenerate singlets $\left|{ }^{1} E_{\theta}\right\rangle$ and $\left|{ }^{1} E_{\epsilon}\right\rangle$ (which form, of course, an orbital doublet), as well as between the above singlets and the top singlet $\left|{ }^{1} A_{1}\right\rangle$. We emphasize that the simplified Hubbard-like form of electron-electron interactions (2.3) which uses two parameters, $U$ and $J_{H}$, in this case is an exact representation of the Coulomb interaction in the $t_{2 g}^{6} e_{g}^{2}$ configuration as obtained in the theory of multiplet spectra, and one finds a one-to-one correspondence between the energies calculated above, and those found with the Racah parameters $A, B$, and $C,{ }^{51}$

$$
\begin{gathered}
E\left({ }^{3} A_{2}\right)=A-8 B, \\
E\left({ }^{1} E\right)=A+2 C, \\
E\left({ }^{1} A_{1}\right)=A+8 B+4 C .
\end{gathered}
$$

Thus the parameters used by us are $U=A+2 C$ and $J_{H}$ $=8 B+2 C .{ }^{50} \mathrm{We}$ normalize the energies by the Coulomb interaction $U$, and introduce

$$
\eta \equiv J_{H} / U
$$




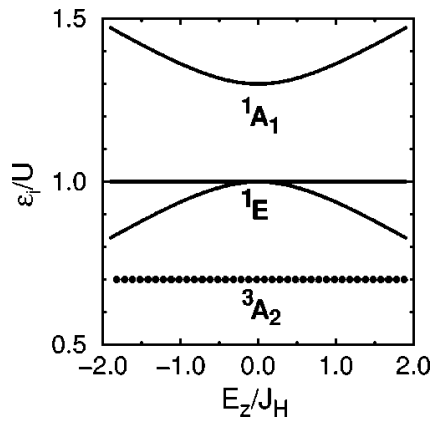

FIG. 2. Energies of the virtual excitations $\varepsilon_{i} / U$ shown in Fig. 1 as functions of $E_{z} / J_{H}$ for $J_{H} / U=0.3$. The lowest triplet $\left|{ }^{3} A_{2}\right\rangle$ state is indicated by full circles, and the singlet states $\left(\left|{ }^{1} E\right\rangle\right.$ and $\left.\left|{ }^{1} A_{1}\right\rangle\right)$ by full lines.

as an energy unit for the Hund's rule exchange interaction. This gives the excitation energies which correspond to the local excitations $d_{i}^{9} d_{j}^{9} \rightarrow d_{i}^{10} d_{j}^{8}$ on a given bond $(i j)$,

$$
\begin{gathered}
\varepsilon\left({ }^{3} A_{2}\right)=1-\eta, \\
\varepsilon\left({ }^{1} E_{\epsilon}\right)=1, \\
\varepsilon\left({ }^{1} E_{\theta}\right)=1+\frac{1}{2} \eta-\frac{1}{2} \eta\left[1+\left(E_{z} / J_{H}\right)^{2}\right]^{1 / 2}, \\
\varepsilon\left({ }^{1} A_{1}\right)=1+\frac{1}{2} \eta+\frac{1}{2} \eta\left[1+\left(E_{z} / J_{H}\right)^{2}\right]^{1 / 2},
\end{gathered}
$$

shown in Fig. 2. We note that the deviation from the equidistant spectrum at $E_{z}=0$ becomes significant only for $\left|E_{z}\right| / J_{H}>1$. Taking the realistic parameters of the cuprates, ${ }^{28}$ one finds for $\mathrm{La}_{2} \mathrm{CuO}_{4}$ with $E_{z}=0.64 \mathrm{eV}$ that $E_{z} / J_{H}$ $\simeq 0.27$, a value representative for systems that are already far from orbital degeneracy. Since we are interested here in what happens close to orbital degeneracy, this allows us to neglect the $E_{z}$ dependence of the energies of the excited $d^{8}$ states, and use the atomic spectrum (2.6) in the derivation presented in Appendix A.

Following the above procedure, we have derived the effective Hamiltonian $\mathcal{H}$ in spin-orbital space,

$$
\mathcal{H}=\mathcal{H}_{J}+\mathcal{H}_{\tau},
$$

where the superexchange part $\mathcal{H}_{J}$ can be most generally written as follows (a simplified form was discussed recently in Ref. 6),

$$
\begin{aligned}
\mathcal{H}_{J}= & \sum_{\langle i j\rangle}\left\{-\frac{t^{2}}{\varepsilon\left({ }^{3} A_{2}\right)}\left(\vec{S}_{i} \cdot \vec{S}_{j}+\frac{3}{4}\right) \mathcal{P} \underset{\langle i j\rangle}{\zeta \xi}\right. \\
& +\frac{t^{2}}{\varepsilon\left({ }^{1} E_{\epsilon}\right)}\left(\vec{S}_{i} \cdot \vec{S}_{j}-\frac{1}{4}\right) \mathcal{P}{ }_{\langle i j\rangle}^{\zeta \xi} \\
& \left.+\left[\frac{t^{2}}{\varepsilon\left({ }^{1} E_{\theta}\right)}+\frac{t^{2}}{\varepsilon\left({ }^{1} A_{1}\right)}\right]\left(\vec{S}_{i} \cdot \vec{S}_{j}-\frac{1}{4}\right) \mathcal{P} \zeta_{i j\rangle}^{\zeta \xi}\right\} .
\end{aligned}
$$

Here $\vec{S}_{i}$ refers to a spin $S=1 / 2$ at site $i$, and $\mathcal{P}_{\langle i j\rangle}^{\alpha \beta}$ are projection operators on the orbital states for each bond,

$$
\begin{gathered}
\mathcal{P} \sum_{\langle i j\rangle}^{\zeta \xi}=\left(\frac{1}{2}+\tau_{i}^{c}\right)\left(\frac{1}{2}-\tau_{j}^{c}\right)+\left(\frac{1}{2}-\tau_{i}^{c}\right)\left(\frac{1}{2}+\tau_{j}^{c}\right), \\
\mathcal{P}_{\langle i j\rangle}^{\zeta \zeta}=2\left(\frac{1}{2}-\tau_{i}^{c}\right)\left(\frac{1}{2}-\tau_{j}^{c}\right) .
\end{gathered}
$$

They are either parallel $\left(P_{i \zeta}=\frac{1}{2}-\tau_{i}^{c}\right)$ to the direction of the bond $\langle i j\rangle$ on site $i$, and perpendicular $\left(P_{j \xi}=\frac{1}{2}+\tau_{j}^{c}\right)$ on the other site $j$, or parallel on both sites, respectively, and are constructed with the following orbital operators associated with the three cubic axes $(a, b, c)$,

$$
\begin{gathered}
\tau_{i}^{a}=-\frac{1}{4}\left(\sigma_{i}^{z}-\sqrt{3} \sigma_{i}^{x}\right), \\
\tau_{i}^{b}=-\frac{1}{4}\left(\sigma_{i}^{z}+\sqrt{3} \sigma_{i}^{x}\right), \\
\tau_{i}^{c}=\frac{1}{2} \sigma_{i}^{z} .
\end{gathered}
$$

The $\sigma$ 's are Pauli matrices acting on the orbital pseudospins

$$
|x\rangle=\left(\begin{array}{l}
1 \\
0
\end{array}\right), \quad|z\rangle=\left(\begin{array}{l}
0 \\
1
\end{array}\right) .
$$

Hence we find a Heisenberg Hamiltonian for the spins, coupled into an orbital problem. While the spin problem is described by the continuous symmetry group $S U(2)$, the orbital problem is clock-model-like, i.e., there are three directional orbitals: $3 x^{2}-r^{2}, 3 y^{2}-r^{2}$, and $3 z^{2}-r^{2}$, but they are not independent. The orbital basis consists of one directional orbital and its orthogonal counterpart, and we have chosen here $|z\rangle \equiv 3 z^{2}-r^{2}$ and $|x\rangle \equiv x^{2}-y^{2}$ orbitals.

In general, the energies of these two orbital states, $|x\rangle$ and $|z\rangle$, are different, and thus the complete effective Hamiltonian of the $d^{9}$ model (2.9) includes as well the crystal-field term (2.4) which we write as

$$
\mathcal{H}_{\tau}=-E_{z} \sum_{i} \tau_{i}^{c} .
$$

Here $E_{z}$ is a crystal field which acts as a "magnetic field" for the orbital pseudospins, and is loosely associated with an uniaxial pressure along the $c$ axis. The $d^{9}$ spin-orbital model (2.9) depends thus on two parameters: (i) the crystal-field splitting $E_{z}$, and (ii) the Hund's rule exchange $J_{H}$.

While the first two terms in Eq. (2.10) cancel for the magnetic interactions in the limit of $\eta \rightarrow 0$, the last term favors AF spin orientation. Although the form (2.10) might in principle be used for further analysis, we prefer to make an expansion of the excitation energies $\varepsilon_{n}$ in the denominators of Eq. (2.10) in terms of $J_{H}$, and use $\eta=J_{H} / U$ [Eq. (2.7)] as a parameter which quantifies the Hund's rule exchange. This results in the following form of the effective exchange Hamiltonian in the $d^{9}$ model (2.9): ${ }^{6,59}$

$$
\begin{aligned}
\mathcal{H}_{J} \simeq & J \sum_{\langle i j\rangle}\left[2\left(\vec{S}_{i} \cdot \vec{S}_{j}-\frac{1}{4}\right) \mathcal{P} \mathcal{P}_{\langle i j\rangle}^{\zeta \xi}-\mathcal{P} \mathcal{P}_{\langle i j\rangle}^{\zeta \xi}\right] \\
& -J \eta \sum_{\langle i j\rangle}\left[\vec{S}_{i} \cdot \vec{S}_{j}\left(\mathcal{P}_{\langle i j\rangle}^{\zeta \zeta}+\mathcal{P} \mathcal{P}_{\langle i j\rangle}^{\zeta \xi}\right)+\frac{3}{4} \mathcal{P} \mathcal{P}_{\langle i j\rangle}^{\zeta \xi}-\frac{1}{4} \mathcal{P} \mathcal{\langle i j \rangle} \zeta .\right.
\end{aligned}
$$

The first term in Eq. (2.14) describes the AF superexchange $\propto J=t^{2} / U$ (where $t$ is the hopping between $|z\rangle$ orbit- 
als along the $c$ axis), and is obtained when the splittings between different excited $d^{8}$ states $\sim J_{H}$ (Fig. 2) are neglected. As we show below, in spite of the AF superexchange $\propto J$, no LRO can stabilize in a system described by the spin-orbital model (2.9) in the limit $\eta \rightarrow 0$ at orbital degeneracy $\left(E_{z}=0\right)$ because of the presence of the frustrating orbital interactions which gives a highly degenerate classical ground state. We emphasize that even in the limit of $J_{H}$ $\rightarrow 0$ the present Kugel-Khomskii model does not obey SU(4) symmetry, essentially because of the directionality of the $e_{g}$ orbitals. Therefore such an idealized SU(4)-symmetric model $^{60}$ does not correspond to the realistic situation of degenerate $e_{g}$ orbitals and is expected to give different answers concerning the interplay of spin and orbital ordering in cubic crystals.

Taking into account the multiplet splittings, we obtain [second line of Eq. (2.14)] again a Heisenberg-like Hamiltonian for the spins coupled into an orbital problem, with a reduced interaction $\propto J \eta$. It is evident that the new terms support FM rather than AF spin interactions for particular orbital orderings. This net FM superexchange originates from the virtual transitions which involve the triplet state $\left|{ }^{3} A_{2}\right\rangle$, which has the lowest energy and thus gives the strongest effective coupling. We remark in passing that the FM channel is additionally enhanced for $d^{4}$ ions when the virtual excitations to double occupancies in $e_{g}$ orbitals happen in the presence of partly filled $t_{2 g}$ orbitals in high-spin configurations, as realized in the manganites. ${ }^{16,17}$

The important feature of the spin-orbital model (2.9) is that the actual magnetic interactions depend on the orbital pattern. This follows essentially from the hopping matrix elements in $H_{\text {kin }}$ (2.2) being different between a pair of $|x\rangle$ orbitals, between a pair of different orbitals (one $|x\rangle$ and one $|z\rangle$ orbital), and between a pair of $|z\rangle$ orbitals, respectively, and depending on the bond direction either in the $(a, b)$ planes, or along the $c$ axis. ${ }^{18}$ We show in Sec. III that this leads to a particular competition between magnetic and orbital interactions, and the resulting phase diagram contains a rather large number of classical phases, stabilized for different values of $E_{z}$ and $J_{H}$.

\section{MEAN-FIELD PHASE DIAGRAM}

\section{A. Anisotropy of antiferromagnetic interactions}

We start the analysis of the $d^{9}$ spin-orbital (or KugelKhomskii) model (2.9)-(2.14) by analyzing the MF solution obtained by replacing the scalar products $\vec{S}_{i} \cdot \vec{S}_{j}$ by the Ising term $S_{i}^{z} S_{j}^{z}$. The MF Hamiltonian may be written for the more general situation where the interaction has uniaxial anisotropy along the $c$ direction in the 3D lattice as follows:

$$
\begin{aligned}
\mathcal{H}_{\mathrm{MF}} \simeq & \sum_{\langle i j\rangle} J_{\alpha}\left[2\left(S_{i}^{z} S_{j}^{z}-\frac{1}{4}\right) \mathcal{P}_{\langle i j\rangle}^{\zeta \zeta}-\mathcal{P} \mathcal{P}_{\langle i j\rangle}^{\zeta \xi}\right] \\
& -\eta \sum_{\langle i j\rangle} J_{\alpha}\left[S_{i}^{z} S_{j}^{z}\left(\mathcal{P} \mathcal{\zeta i j \rangle}_{\langle i j}^{\zeta \zeta}+\mathcal{P} \mathcal{P}_{\langle i j\rangle}^{\zeta \xi}\right)+\frac{3}{4} \mathcal{P} \sum_{\langle i j\rangle}^{\zeta \xi}\right. \\
& \left.-\frac{1}{4} \mathcal{P} \underset{\langle i j\rangle}{\zeta \zeta}\right]-E_{z} \sum_{i} \tau_{i}^{c},
\end{aligned}
$$

where $J_{a}=J_{b}=J$ and $J_{c}=J \beta$. For $\beta>1$ the nearestneighbor bonds $\langle i j\rangle \| c$ are shorter, while for $\beta<1$ these bonds are longer than the bonds within the $(a, b)$ planes. In the limit of $\beta \rightarrow 0$ the bonds along the $c$ axis may be neglected and the model reduces to a $2 \mathrm{D}$ model, representative for the magnetic interactions between $\mathrm{Cu}$ ions within the $\mathrm{CuO}_{2}$ planes of the high-temperature superconductors.

The presence of AF spin interactions $\propto J$ suggests magnetic superstructures with staggered magnetization, and we considered several possibilities, with two- and four-sublattice $3 \mathrm{D}$ structures, giving rise to G-AF and A-AF phases, AF 1D chains coupled ferromagnetically, and others. The MF Hamiltonian contains as well an AF interaction between orbital variables, $\sim J \tau_{i}^{\alpha} \tau_{j}^{\alpha}$, which suggests that it might be energetically more favorable to alternate the orbitals in a certain regime of parameters, and pay thereby part of the magnetic energy. This illustrates the essence of the frustration of the magnetic interactions present in the spin-orbital model (2.9), as discussed in Sec. I. Therefore for any classical state the orbitals occupied by the holes have to be optimized, and we allowed MO states,

$$
|i \mu \sigma\rangle=\cos \theta_{i}|i z \sigma\rangle+\sin \theta_{i}|i x \sigma\rangle,
$$

with the values of the mixing angles $\left\{\theta_{i}\right\}$ being variational parameters to be found from the minimization of the classical energy.

The superexchange in Eq. (3.1) depends strongly on the orbital state. At large positive $E_{z}$, where the crystal field strongly favors $|x\rangle$ occupancy over $|z\rangle$ occupancy, one expects that $\theta_{i}=\pi / 2$ in Eq. (3.2), and the holes occupy $|x\rangle$ orbitals on every site. In this case the spins do not interact in the $c$ direction (see Fig. 1), and there is also no orbital energy contribution. Hence the $(a, b)$ planes will decouple magnetically, while within each plane the superexchange is AF and equal to $9 J / 4$ along $a$ and $b$. These interactions stabilize a 2D antiferromagnet, called further AFxx. The resulting 2D Néel state with decoupled $(a, b)$ planes along the $c$ direction is the well-known classical ground state of the high- $T_{c}$ superconductors $\mathrm{La}_{2} \mathrm{CuO}_{4}$ and $\mathrm{YBa}_{2} \mathrm{Cu}_{3} \mathrm{O}_{6} \cdot{ }^{61} \mathrm{In}$ contrast, if $E_{z}<0$ and $\left|E_{z}\right|$ is large, $\left|E_{z}\right| / J \gg 1$, then $\theta_{i}=0$ in Eq. (3.2), and the holes occupy $|z\rangle$ orbitals. The spin system has then strongly anisotropic AF superexchange, being $4 J$ between two $|z\rangle$ orbitals along the $c$ axis, and $J / 4$ between two $|z\rangle$ orbitals in the $(a, b)$ planes, respectively. The corresponding 3D Néel state with holes occupying $|z\rangle$ orbitals is called AFzz. The spin and orbital order in both AF phases is shown schematically within the $(a, b)$ planes in Fig. 3 .

\section{B. Antiferromagnetic states in the 3D model}

Assuming an AF classical order in all three directions, the so-called G-AF state, it is thus obvious that for large $\left|E_{z}\right|$ one finds either the AFxx or the AFzz phase, depending on 


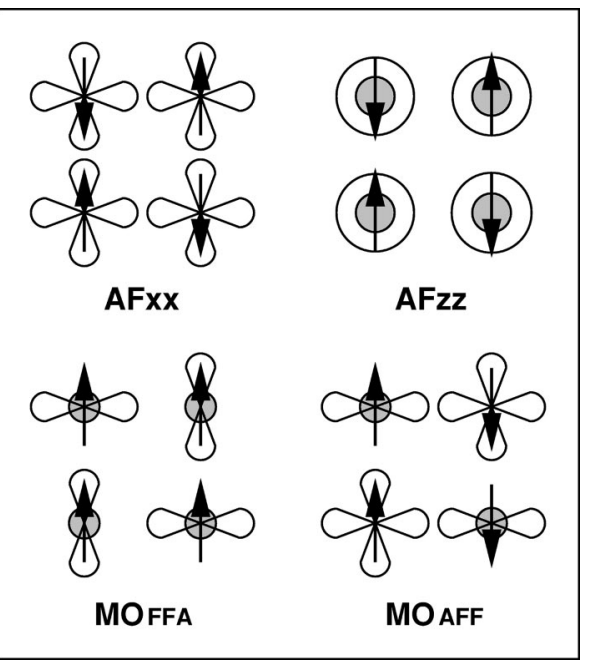

FIG. 3. Schematic representation of orbital and magnetic longrange order within the $(a, b)$ planes of $\mathrm{AFxx}$ (with $|x\rangle$ orbitals occupied), AFzz (with $|z\rangle$ orbitals occupied), MOFFA [with mixed orbitals and FM (AF) order along $a$ and $b$ axis ( $c$ axis)], and MOAFF phases (with the orbitals as in MOFFA, but rotated to give AF interaction along the $a$ axis), respectively. The shaded parts of different orbitals are oriented along the $c$ axis. The spins (arrows) in the next $(a, b)$ plane in the $c$ direction are AF to those below them in AFzz and MOFFA phases, and FM in MOAFF phase. In the AFxx phase there is no magnetic coupling to the next plane along the $c$ axis, but this degeneracy is removed in MOAAF phase, where a small $|z\rangle$ component promotes a FM coupling.

whether $E_{z}>0$ or $E_{z}<0$, with the following energies normalized per one site,

$$
\begin{gathered}
E_{\mathrm{AFxx}}=-3 J\left(1-\frac{\eta}{4}\right)-\frac{1}{2} E_{z}, \\
E_{\mathrm{AFzz}}=-J\left(1+\frac{\eta}{4}\right)-2 J \beta\left(1-\frac{\eta}{2}\right)+\frac{1}{2} E_{z} .
\end{gathered}
$$

The AFxx and AFzz phases are degenerate in a 3D system $(\beta=1)$ along the line $E_{z}=0$, while decreasing $\beta$ moves the degeneracy to negative values of $E_{z}$, namely to $E_{z}$ $=-2 J(1-\beta)(1-\eta / 2)$.

However, for intermediate values of $\left|E_{z}\right|$ one should allow for mixed orbitals. Following the argument above about the AF nature of the orbital interaction, we assume alternating orbitals at two sublattices, $A$ and $B$. The alternation should allow the orbitals to compromise between being identical (optimizing the magnetic energy) and being orthogonal (optimizing the orbital energy). This is realized by choosing in Eq. (3.2) the angles alternating between the sublattices: $\theta_{i}=+\theta$ for $i \in A$, and $\theta_{j}=-\theta$ for $j \in B$, respectively;

$$
\begin{aligned}
& |i \mu \sigma\rangle=\cos \theta|i z \sigma\rangle+\sin \theta|i x \sigma\rangle, \\
& |j \mu \sigma\rangle=\cos \theta|j z \sigma\rangle-\sin \theta|j x \sigma\rangle .
\end{aligned}
$$

The calculation of the energy can be performed either by evaluating the average values of the operator variables $\left\{\tau_{i}^{\alpha}\right\}$, or by taking the average values of the orbital projection operators $\left\{P_{i \alpha}\right\}$ as given in Eq. (A3). Using the two-sublattice orbital ordering (3.4), one finds for the bonds $\langle i j\rangle \|(a, b)$

$$
\begin{gathered}
\left\langle P_{i \xi} P_{j \zeta}+P_{i \zeta} P_{j \xi}\right\rangle=\frac{1}{8}\left(7-4 \cos ^{2} 2 \theta\right), \\
\left\langle 2 P_{i \zeta} P_{j \zeta}\right\rangle=\frac{1}{8}(1-2 \cos 2 \theta)^{2},
\end{gathered}
$$

and for the bonds $\langle i j\rangle \|_{c}$

$$
\begin{gathered}
\left\langle P_{i x} P_{j z}+P_{i z} P_{j x}\right\rangle=\frac{1}{2}\left(1-\cos ^{2} 2 \theta\right), \\
\left\langle 2 P_{i z} P_{j z}\right\rangle=\frac{1}{2}(1+\cos 2 \theta)^{2} .
\end{gathered}
$$

The classical energy per site as a function of $\theta$ is then given by

$$
\begin{aligned}
E(\theta)= & -\frac{J}{4}\left(1+\frac{\eta}{2}\right)\left(7-4 \cos ^{2} 2 \theta\right) \\
& -\frac{J}{4}\left(1-\frac{\eta}{2}\right)(1-2 \cos 2 \theta)^{2} \\
& -\frac{J}{2} \beta\left(1+\frac{\eta}{2}\right)\left(1-\cos ^{2} 2 \theta\right) \\
& -\frac{J}{2} \beta\left(1-\frac{\eta}{2}\right)(1+\cos 2 \theta)^{2} \\
& +\frac{1}{2} E_{z} \cos 2 \theta
\end{aligned}
$$

This has a minimum at

$$
\cos 2 \theta=-\frac{\left(1-\frac{\eta}{2}\right)(1-\beta)+\frac{1}{2} \varepsilon_{z}}{(2+\beta) \eta},
$$

where $\varepsilon_{z}=E_{z} / J$, if $\eta \neq 0$, and provided that $|\cos 2 \theta| \leqslant 1$ (a similar condition applies to all the other states with MO considered below). So, as long as $2 J(\beta-1)-3 J(\beta+1) \eta \leqslant E_{z}$ $\leqslant 2 J(\beta-1)+J(5+\beta) \eta$, there is genuine MO order, while upon reaching the smaller (larger) boundary value for $E_{z}$, the orbitals go over smoothly into $|z\rangle(|x\rangle)$, i.e., one retrieves the AFzz (AFxx) phase. Taking the magnetic ordering in the three cubic directions $[a b c]$ as a label to classify the classical phases with MO (3.4), we call the phase obtained in the regime of genuine MO order MOAAA, with classical energy given by

$$
E_{\mathrm{MOAAA}}=-\left(2+\beta+\frac{3}{4} \eta\right) J-J \frac{\left[(2-\eta)(1-\beta)+\varepsilon_{z}\right]^{2}}{4(2+\beta) \eta} .
$$

Upon increasing $J_{H}$, the FM interactions occur which increase the energy of the AF phases in three dimensions by the term $\frac{3}{4} \eta$ per site in Eqs. (3.3) (a similar increase of energy occurs also in the MOAAA phase in the region of its existence). This indicates frustration of magnetic interactions and opens a potential possibility that other classical phases with FM order along particular directions might be more stable. We have found a few classical phases when the spins order ferromagnetically either in particular planes, or along one spatial direction, and this magnetic order coexists with MO occupied by holes.

For example, the angles in Eq. (3.2) can be chosen in such a way that at least one of the orbitals on two neighboring sites is perpendicular to the bond direction, e.g., is like $y^{2}$ 
$-z^{2}$ type for a bond along the $a$ axis. In such a case, the AF superexchange vanishes, and one finds instead a weaker FM interaction, in agreement with the Goodenough-Kanamori rules. ${ }^{32}$ By this mechanism Kugel and Khomskii ${ }^{5}$ proposed an alternating orbital order to explain the FM planes observed in $\mathrm{KCuF}_{3}$. Following this argument, let us assume FM order within $(a, b)$ planes, and the same form (3.4) as above for the alternating orbitals at the two sublattices $A$ and $B$. As alternating orbitals can only be arranged to be perpendicular to the bonds in at most two spatial directions, such an arrangement for the $(a, b)$ planes forces the orbitals to have nonzero lobes along $c$. This results in sizable AF superexchange for the bonds $\langle i j\rangle$ parallel to $c$, which will order the spins antiferromagnetically in the $c$ direction. The orbitals may either repeat or stagger along the $c$ axis, and both states give the same mean-field energy. Taking the magnetic ordering in the three cubic directions $[a b c]$ as a label to classify the classical phases with MO (3.4), we call this ground state the MOFFA phase. With the help of Eqs. (3.5) and (3.6) one obtains the following classical energy as a function of $\theta$ :

$$
\begin{aligned}
E(\theta)= & -\frac{J}{4}(1+\eta)\left(7-4 \cos ^{2} 2 \theta\right) \\
& -\frac{J}{2} \beta\left(1+\frac{\eta}{2}\right)\left(1-\cos ^{2} 2 \theta\right) \\
& -\frac{J}{2} \beta\left(1-\frac{\eta}{2}\right)(1+\cos 2 \theta)^{2} \\
& +\frac{1}{2} E_{z} \cos 2 \theta,
\end{aligned}
$$

with a minimum at

$$
\cos 2 \theta=\frac{\beta\left(1-\frac{\eta}{2}\right)-\frac{1}{2} \varepsilon_{z}}{2+(2+\beta) \eta},
$$

where again the MO exist as long as $|\cos 2 \theta| \leqslant 1$. Using Eqs. (3.10) and (3.11) one finds that the classical energy of the MOFFA phase is given by

$$
E_{\mathrm{MOFFA}}=-\frac{J}{4}(11-7 \eta)-\frac{J}{2} \frac{\left[\beta\left(1-\frac{\eta}{2}\right)-\frac{1}{2} \varepsilon_{z}\right]^{2}}{2+(2+\beta) \eta} .
$$

As a special case, let us consider first degenerate orbitals $\left(E_{z}=0\right)$ in a $3 \mathrm{D}$ system $(\beta=1)$. Equation (3.11) simplifies in this case to $\cos 2 \theta=(1-\eta / 2) /(2+3 \eta)$. A particularly simple result is found at $\eta=0$ where $\cos 2 \theta=1 / 2$, i.e., $\theta$ $=\pi / 6$, and the orbitals stagger like $x^{2}-z^{2}$ and $y^{2}-z^{2}$, as shown in Fig. 3. This staggering was proposed by Kugel and Khomskii as a ground state of $\mathrm{KCuF}_{3} ;{ }^{9}$ of course, this state is not realized for the realistic parameters with $\eta \simeq 0.3$, but the optimized orbitals with $\theta$ given by Eq. (3.11) are not so far from this idealized picture.

The energy of the MOFFA phase is degenerate with that of the AF phases at the classical degeneracy point, $M$ $\equiv\left(E_{z} / J, \eta\right)=(0,0)$, and this phase becomes more stable at $\eta>0$ and $E_{z} / J \simeq 0$. The magnetic energy is gained due to relatively strong $\mathrm{AF}$ interactions on the bonds $\langle i j\rangle \| c$, and weak FM interactions in the planes $(a, b)$, perpendicular to the preferred directionality of the MO (3.2) along the $c$ direction, while the orbital energy is gained due to orbital alternation within the $(a, b)$ planes. Such orbital ordering remains stable with decreasing $E_{z}<0$, while two similar states with the staggering either within the $(b, c)$ or the $(a, c)$ planes, are more stable for $E_{z}>0$. Following our convention, these two degenerate MO states stable at $E_{z}>0$ are called MOAFF and MOFAF (see Fig. 3), respectively. However, the MO involve in this case the directional orbital $|\zeta\rangle$ along the AF bonds (i.e., $\left|\zeta_{a}\right\rangle \sim 3 x^{2}-r^{2}$ for MOAFF or $\left|\zeta_{b}\right\rangle$ $\sim 3 y^{2}-r^{2}$ for MOFAF, respectively), and the corresponding orthogonal orbital, $|\xi\rangle$. Therefore, since the symmetrybreaking field acts on $|z\rangle$ orbitals, the angles in the two sublattices cannot be exactly equivalent in this case, unlike in the MOFFA phase, and we adopted an ansatz,

$$
\begin{aligned}
& |i \sigma\rangle=\cos \theta_{+}|i \xi \sigma\rangle+\sin \theta_{+}|i \zeta \sigma\rangle, \\
& |j \sigma\rangle=\cos \theta_{-}|i \xi \sigma\rangle-\sin \theta_{-}|i \zeta \sigma\rangle,
\end{aligned}
$$

where $i \in A, j \in B$, and $\theta_{ \pm}>0$ for the two sublattices. Introducing for convenience the new angles, $\phi=\frac{1}{2}\left(\theta_{+}+\theta_{-}\right)$, and $\delta=\theta_{+}-\theta_{-}$, one finds the following conditions for the energy minimum of the classical MOAFF phase,

$$
\begin{aligned}
\cos 2 \phi= & -\frac{1}{4}\left\{\left[(1+\beta)(2-\eta)+\varepsilon_{z}\right] \cos \delta+\sqrt{3} \varepsilon_{z} \sin \delta\right\} \\
& \times[1+\beta+(1+2 \beta) \eta]^{-1} \\
\tan 2 \delta= & \frac{1}{2} \sqrt{3}\left[(1+\beta)(2-\eta)+\varepsilon_{z}\right] \varepsilon_{z} \\
& \times\left\{4[1+\beta+(1+2 \beta) \eta]+\left[(1+\beta)(2-\eta)+\varepsilon_{z}\right]^{2}\right. \\
& \left.-\frac{3}{4} \varepsilon_{z}^{2}\right\}^{-1},
\end{aligned}
$$

and the energy is given by

$$
\begin{aligned}
E_{\mathrm{MOAFF}}= & -\frac{J}{4}[7(1+\eta)+2 \beta(1+\cos \delta)] \\
& -\frac{J}{32} \frac{\left\{\left[(1+\beta)(2-\eta)+\varepsilon_{z}\right] \cos \delta+\sqrt{3} \varepsilon_{z} \sin \delta\right\}^{2}}{1+\beta+(1+2 \beta) \eta} .
\end{aligned}
$$

Finally, one may consider states in which magnetic energy is gained in the $c$ direction due to MO with a small admixture of $|z\rangle$ into orbitals of predominantly $|x\rangle$ character, i.e., $\sin \theta_{i}=1-\epsilon$ in Eq. (3.2). As such a state is a modification of the AFxx phase, the two sublattices in the $(a, b)$ planes are again physically equivalent, and it suffices to introduce a single angle $\theta$ to characterize this state. Apart from (large) energy contributions due to AF order on the bonds in the $(a, b)$ planes, the expansion of the ground-state energy contains also (small) terms depending on the spin order in the $c$ direction, $\left\langle S_{i}^{z} S_{j}^{z}\right\rangle_{\| c}$,

$$
E=(1+\cos 2 \theta)(1+\cos 2 \theta-\eta)\left\langle S_{i}^{z} S_{j}^{z}\right\rangle_{\| c}+\text { const, }
$$

which prefers FM order as long as $(1+\cos 2 \theta)<\eta$. The reason is that the AF superexchange is a fourth-order effect 


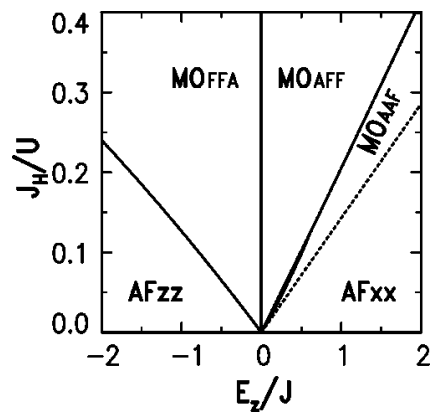

FIG. 4. Mean-field phase diagram of the 3D spin-orbital model (2.9) in the $\left(E_{z}, J_{H}\right)$ plane $(\beta=1)$. The lines separate the classical states shown in Fig. 3; the transition from AFxx to MOAFF phase is second order (dashed line), while the remaining transitions are first order (full lines).

$\sim \epsilon^{4}$, while the FM interactions $\propto \eta$ are second order, $\sim \epsilon^{2}$, and give a lower energy $E$ as long as the $|z\rangle$ occupancy is small enough. Following our convention, we call the resulting state the MOAAF phase, with the mixing angle given by

$$
\cos 2 \theta=-\frac{1-\frac{\eta}{2}+\frac{1}{2} \varepsilon_{z}}{\beta(1+\eta)+2 \eta},
$$

and the classical energy by

$$
\begin{aligned}
E_{\mathrm{MOAAF}}= & -\left(2+\frac{3}{4} \eta\right) J-\frac{1}{2} \beta(1+\eta) \\
& -J \frac{\left(2-\eta+\varepsilon_{z}\right)^{2}}{2[\beta(1+\eta)+2 \eta]} .
\end{aligned}
$$

Therefore only when the average population of the $|z\rangle$ orbitals, $\sim \cos ^{2} \theta$, increases sufficiently, one can find a transition to the AF phase with mixed orbitals, MOAAA, discussed above.

By making several other choices of orbital mixing and classical magnetic order, we have verified that no other commensurate ordering with up to four sublattices can be stable in the present situation. Although some other phases could be found, they were degenerate with the above phases only at the $M$ point, and otherwise had higher energies. Thus we obtain the classical phase diagram of the 3D spin-orbital model (2.9) by comparing the energies of the six above phases for various values of two parameters, $\left\{E_{z} / J, J_{H} / U\right\}$ : two AF phases with two sublattices and pure orbital character (AFxx and AFzz), three A-AF phases with four sublattices (MOFFA and two degenerate phases: MOAFF and MOAFF), one C-AF phase (MOAAF), and one G-AF phase with MO's (MOAAA). While the orbital mixing is unstable at $\eta=0$, the generic sequence of classical phases at finite $\eta$ and decreasing $E_{z} / J$ is: AFxx, MOAAF, MOAAA, MOAFF, MOFFA, and AFzz, and the magnetic order is tuned together with the gradually increasing $|z\rangle$ character of the occupied orbitals.

The result for cubic symmetry $(\beta=1)$ is presented in Fig. 4 , where one finds all six phases, but the MOAAA phase does stabilize only in a very restricted regime of parameters with $J_{H} / U<0.1$, before MOAFF takes over. Only the first of the above transitions is a continuous one, and the $|z\rangle$
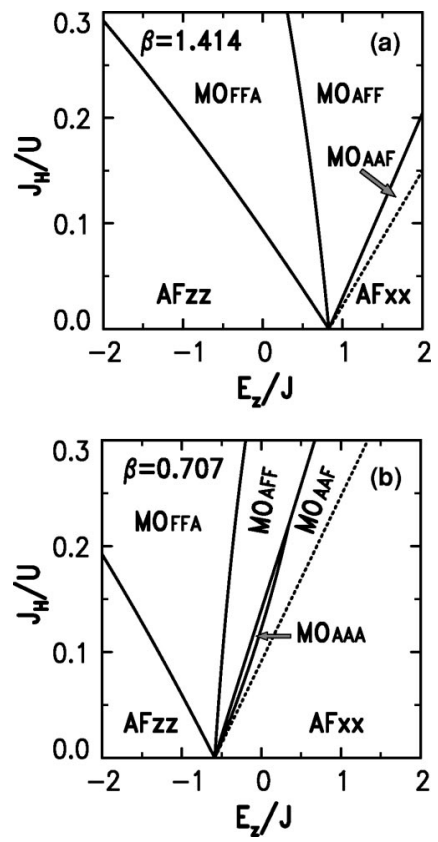

FIG. 5. Mean-field phase diagrams of the spin-orbital model (2.9) in the $\left(E_{z}, J_{H}\right)$ plane for different values of hopping along the $c$ axis: (a) $\beta=1.414$, and (b) $\beta=0.707$. The magnetic phases and lines are as in Fig. 4.

amplitude $\sim \cos ^{2} \theta$ increases smoothly from zero and removes the built-in degeneracy of the 2D AFxx phase with respect to the magnetic order along the $c$ direction. All the other transition lines in Fig. 4 are associated with jumps in the magnetic and in orbital patterns. We emphasize that all the considered phases with magnetic LRO are degenerate at the point $M$, with classical energy of $-3 J$. In fact, $M$ is an infinite-order quantum critical point, since not only may the spins be chosen to be FM in certain planes, whence the orbitals have to be tuned to compensate the loss of the magnetic energy by the orbital energy contributions, as realized in all MO phases, but also may the orbitals be rotated freely when the spins are AF in all three directions. We note, however, that the magnetic terms are essential, and in a purely disordered spin system, with $\left\langle S_{i}^{z} S_{j}^{z}\right\rangle=0$, a higher energy of $-21 J / 8$ is found even with the optimal choice of orbitals with $\cos 2 \theta=0$.

The symmetry with respect to $E_{z}=0$ is explicitly broken in the phase diagram of Fig. 4. The crucial point is that the orbitals favored by nonzero $E_{z}$ have different directionality: unidirectional $(|z\rangle)$ for $E_{z}<0$, planar $(|x\rangle)$ for $E_{z}>0$. For the G-AF phases this leads straightforwardly to different exchange interactions depending on which orbital is occupied. A similar asymmetry is also found for the MO phases, and it is for this reason that an additional MOAAF phase, with FM chains along the $c$ axis is found only for $E_{z}>0$. By contrast, we note that the phase diagram is invariant under a change of the basis orbitals to $3 x^{2}-r^{2}$ and $y^{2}-z^{2}$ and a simultaneous rotation of the crystal field to a situation where the new orbitals are split by a crystal-field parameter $E_{\zeta}$, having an analogous meaning to $E_{z}$. This demonstrates the full cubic symmetry of the present Hamiltonian, but this symmetry is explicitly broken by a uniaxial stress along the $c$ direction, consistent with the $Q_{3}$ static distortions considered by Kanamori. ${ }^{62}$ 


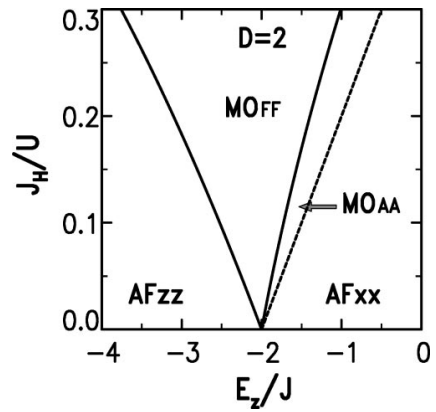

FIG. 6. Mean-field phase diagram of the spin-orbital model (2.9) in the $\left(E_{z}, J_{H}\right)$ plane in two dimensions $(\beta=0)$. Full lines separate the classical states AFxx, AFzz, and MOFF shown in Fig. 3, while the spin order in the MOAA phase is $\mathrm{AF}$, and the orbitals are in between those in AFxx and MOFF phase.

We also investigated the phase diagrams for the case of modified hopping along the $c$ direction $(\beta \neq 1)$. One finds that increased hopping $(\beta=1.414)$ in the $c$ direction stabilizes the MO phases, and in particular the MOAFF (MOFAF) phase [Fig. 5(a)]. By contrast, the MO phases are stable in a narrower range of $E_{z}$ for a fixed value of $J_{H} / U$, if the hopping along the $c$ direction is decreased below $\beta=1$ [an example of $\beta=0.707$ is shown in Fig. 5(b)]. The decreased stability of the MOAFF phase promotes in this case the AF order with MO in the MOAAA phase. The latter phase is stable only in a relatively narrow range of $E_{z}$, and only for small enough $J_{H} / U$; an increase of $J_{H} / U$ favors instead FM order along the $c$ direction. We also note that the orbital mixing sets for the MOAAA phase (3.8) only at a smaller value of $E_{z}$ than in the MOAAF phase (3.18). Interestingly, the point of high degeneracy of the classical states exists independently of the value of $\beta$, and moves for $\beta \neq 1$ to $E_{z}=-2 J(1-\beta)$. This demonstrates the generic nature of the internal frustration of spin and orbital interactions in the model, and the crystal-field term just plays here a compensating role for the missing (or enhanced) magnetic interactions within the $(a, b)$ planes.

Independently of the value of $\beta$, the spin-orbital model (2.9) has a universal feature: different classical spin structures become degenerate at the critical lines in Figs. 4-6. This is also encountered in frustrated 2D magnetic lattices described by simple Heisenberg Hamiltonians ${ }^{42}$ and may thus be regarded as a signature of frustration. However, unlike in the purely spin models, in the present case (2.9), the sign of the interactions changes because of the coupling to the orbital sector, and this reduces the effective dimensionality for the $\mathrm{AF}$ interactions $\sim J$, with the 3D system behaving like a quasi-1D antiferromagnet.

\section{Phase diagram of a 2D model}

As a special case, we considered the limit of $\beta \rightarrow 0$ which gives a $2 \mathrm{D}$ spin-orbital model. The two AF phases with either $|x\rangle$ or $|z\rangle$ orbitals occupied, AFxx and AFzz, are degenerate at $E_{z}=-2 J$. This asymmetry reflects the large difference between the superexchange interactions for $|x\rangle$ and $|z\rangle$ orbitals within the $(a, b)$ planes of a 2D system which has to be compensated by the orbital energy (2.13).

As the presence of FM planes $\| c$ axis is crucial for the ordering in the MOAFF phase (see Fig. 3), this phase disap-

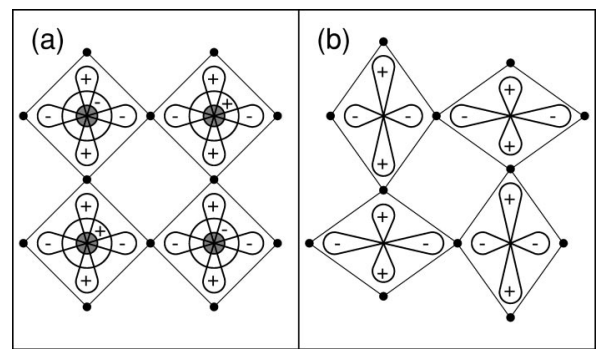

FIG. 7. Schematic representation of the mixed orbitals in $(a, b)$ planes of the MOFF phase in a 2D model: (a) the orbitals with their phases, and (b) the resulting distortion in the oxygen lattice, stabilized by the orbital ordering.

pears, while the remaining two phases with $\mathrm{AF}$ order within $(a, b)$ planes, MOAAA and MOAAF, collapse into a single MOAA phase. Hence one finds in two dimensions a classical phase diagram with only four phases, which are stable with decreasing $E_{z}$ and at finite $\eta$ in the following order: AFxx, MOAA, MOFF, and AFzz (Fig. 6). The 2D phase diagram shows in particular that strong AF superexchange in the $c$ direction is not the stabilizing factor of the MOFFA phase in the 3D model, but instead these phases are stable due to the orbital interactions which enforce the orbital alternation shown in Fig. 3.

For the realistic parameters of $\mathrm{La}_{2} \mathrm{CuO}_{4}$ the $\mathrm{Cu} d_{x^{2}-y^{2}}$ and $d_{3 z^{2}-r^{2}}$ orbitals are split, and $E_{z} \simeq 0.64 \mathrm{eV}{ }^{28}$ This material belongs together with $\mathrm{Nd}_{2} \mathrm{CuO}_{4}$ to the class of cuprates with weakly coupled $\mathrm{CuO}_{2}$ planes, and one finds in the present treatment a 2D AFxx state, as observed in neutron experiments. ${ }^{63}$ If, however, the orbital splitting is small in a 2D situation, the orbital ordering couples strongly to the lattice, as the hybrids with alternating phasing on two sublattices are formed according to Eqs. (3.13) The net result is a quadrupolar distortion as indicated in Fig. 7. In fact, using these arguments Kugel and Khomskii predicted ${ }^{33}$ the existence of such a structural distortion in the MOFF phase of a quasi-2D compound $\mathrm{K}_{2} \mathrm{CuF}_{4}$. This prediction was confirmed experimentally a few years later. ${ }^{64}$

The MOFF phase of $\mathrm{K}_{2} \mathrm{CuF}_{4}$ is magnetically polarized, has no transverse quantum fluctuations, and is thus well described in a classical theory. In the next sections we concentrate ourselves on the 3D case, where the quantum fluctuations are strong and destabilize the classical magnetic ordering in a particular regime of parameters.

\section{ELEMENTARY EXCITATIONS}

\section{A. General formalism}

The presence of the orbital degrees of freedom in the Hamiltonian (2.9) results in excitation spectra that are qualitatively different from those of the HAF with a single spinwave mode. As we have discussed in the limit of $J_{H}=0$, the transverse excitations are twofold: spin-waves and spin-andorbital waves. ${ }^{65}$ In addition to these two modes there are also longitudinal (purely orbital) excitations, and thus one finds three elementary excitations for the present spin-orbital model (2.9). ${ }^{6,65,66}$ This gives therefore the same number of modes as found in a 1D SU(4) symmetric spin-orbital model in the Bethe ansatz method. ${ }^{67,60} \mathrm{We}$ emphasize that this fea- 
ture is a consequence of the dimension (equal to 15) of the so(4) Lie algebra of the local operators, as explained below, and is not related to the global symmetry of the Hamiltonian. Here we present the analysis of the realistic $d^{9}$ spin-orbital model for the 3D simple cubic (i.e., perovskitelike) lattice, using linear spin-wave theory, ${ }^{68,69}$ generalized such as to make it applicable to the present situation.

Before we introduce the excitation operators, it is convenient to rewrite the spin-orbital model (2.9) in a different representation which uses a four-dimensional space, $\{|x \uparrow\rangle,|x \downarrow\rangle,|z \uparrow\rangle,|z \downarrow\rangle\}$, instead of a direct product of the spin and orbital spaces. Hence we introduce operators which define purely spin excitations in individual orbitals,

$$
S_{i x x}^{+}=d_{i x \uparrow}^{\dagger} d_{i x \downarrow}, \quad S_{i z z}^{+}=d_{i z \uparrow}^{\dagger} d_{i z \downarrow},
$$

and operators for simultaneous spin-and-orbital excitations,

$$
K_{i x z}^{+}=d_{i x \uparrow}^{\dagger} d_{i z \downarrow}, \quad K_{i z x}^{+}=d_{i z \uparrow}^{\dagger} d_{i x \downarrow} .
$$

The corresponding $S_{i \alpha \alpha}^{z}$ and $K_{i \alpha \beta}^{z}$ operators are defined as follows,

$$
\begin{gathered}
S_{i x x}^{z}=\frac{1}{2}\left(n_{i x \uparrow}-n_{i x \downarrow}\right), \\
S_{i z z}^{z}=\frac{1}{2}\left(n_{i z \uparrow}-n_{i z \downarrow}\right), \\
K_{i x z}^{z}=\frac{1}{2}\left(d_{i x \uparrow}^{\dagger} d_{i z \uparrow}-d_{i x \downarrow}^{\dagger} d_{i z \downarrow}\right), \\
K_{i z x}^{z}=\frac{1}{2}\left(d_{i z \uparrow}^{\dagger} d_{i x \uparrow}-d_{i z \downarrow}^{\dagger} d_{i x \downarrow}\right) .
\end{gathered}
$$

The Hamiltonian (2.9) contains also purely orbital interactions which can be expressed using the following orbitalflip $\left(T_{i \alpha \beta}\right)$ and orbital-polarization $\left(n_{i-}\right)$ operators,

$$
\begin{gathered}
T_{i x z}=\frac{1}{2}\left(d_{i x \uparrow}^{\dagger} d_{i z \uparrow}+d_{i x \downarrow}^{\dagger} d_{i z \downarrow}\right), \\
T_{i z x}=\frac{1}{2}\left(d_{i z \uparrow}^{\dagger} d_{i x \uparrow}+d_{i z \downarrow}^{\dagger} d_{i x \downarrow}\right), \\
n_{i-}=\frac{1}{2}\left(d_{i x \uparrow}^{\dagger} d_{i x \uparrow}+d_{i x \downarrow}^{\dagger} d_{i x \downarrow}-d_{i z \uparrow}^{\dagger} d_{i z \uparrow}-d_{i z \downarrow}^{\dagger} d_{i z \downarrow}\right) .
\end{gathered}
$$

In order to simplify the notation, we also introduce sum operators for the spin-and-orbital and purely orbital operators,

$$
\begin{gathered}
K_{i}^{+}=K_{i x z}^{+}+K_{i z x}^{+}, \\
K_{i}^{z}=K_{i x z}^{z}+K_{i z x}^{z}, \\
T_{i}=T_{i x z}+T_{i z x} .
\end{gathered}
$$

The full set of local operators at a site $i$ constitute an so(4) Lie algebra. While the spin operators (4.1) fulfill of course for $x$ and $z$ separately the usual su(2) commutation relations, they also form collectively a subalgebra of so(4), and the same holds for the spin-and-orbital operators (4.2). However, as we will see below, for the calculation of the excitations one also needs commutators between spin and spin-andorbital operators, so that one cannot avoid considering the full Lie-algebra structure of so(4), discussed in Appendix B.

The number of collective modes in a particular phase may be determined as follows. The so(4) Lie algebra consists of three Cartan operators, i.e., operators diagonal on the local eigenstates of the symmetry-broken phase under consideration (e.g., $S_{i x x}^{z}, S_{i z z}^{z}$, and $n_{i-}$ in the AFxx phase), plus 12 nondiagonal operators turning the eigenstates into one another (like $S_{i x x}^{+}$and $S_{i z z}^{+}$in AFxx). Out of those twelve operators, six connect two excited states (like $S_{i z z}^{+}$in AFxx), and are physically irrelevant (at the random-phase approximation level), because they give only rise to "ghost" modes, modes for which the spectral function vanishes identically. The remaining six operators connect the local ground state with an excited state, three of them describing an excitation and three a deexcitation, and only these six operators are physically relevant. Out of the three excitations (deexcitations), two are transverse, i.e., change the spin, and one is longitudinal, i.e., does not affect the spin. For a classical phase with $L$ sublattices one therefore has $4 L$ transverse and $2 L$ longitudinal operators per unit cell. Since the spin-orbital Hamiltonian (2.9) does not couple transverse and longitudinal operators, this yields also $4 L$ transverse and $2 L$ longitudinal modes. Because of time-reversal invariance they all occur in pairs with opposite frequencies, $\pm \omega_{\vec{k}}^{(n)}$.

Finally, the $S U(2)$ spin invariance of the Hamiltonian guarantees that the transverse operators raising the spin are decoupled from those lowering the spin, and that they are described by the same set of equations of motion, so that the transverse modes are pairwise degenerate. Such a simplification does not occur in the longitudinal sector. So, in conclusion, in an $L$-sublattice phase there are $L$ doubly-degenerate positive-frequency transverse modes and $L$ nondegenerate positive-frequency longitudinal modes, accompanied by the same number of negative-frequency modes. This may be compared with the well-known situation in the HAF, where there is, with only spin operators involved, only one (not two) doubly-degenerate positive-frequency (transverse) mode in the two-sublattice Néel state.

For the actual evaluation it is convenient to decompose the superexchange terms in the spin-orbital Hamiltonian (2.9),

$$
\mathcal{H}_{J}=\mathcal{H}_{\|}+\mathcal{H}_{\perp}
$$

into two parts which depend on the bond direction:

(i) for the bonds $\langle i j\rangle \|(a, b)$,

$$
\begin{aligned}
\mathcal{H}_{\|}= & \frac{1}{4} J \sum_{\langle i j\rangle \|}\left[\left(1-\frac{1}{2} \eta\right)\left(3 \vec{S}_{i x x}+\vec{S}_{i z z}+\lambda_{i j} \sqrt{3} \vec{K}_{i}\right)\right. \\
& \times\left(3 \vec{S}_{j x x}+\vec{S}_{j z z}+\lambda_{i j} \sqrt{3} \vec{K}_{j}\right)-2 \eta \vec{S}_{i} \cdot \vec{S}_{j}+(1+2 \eta) \\
& \left.\times\left(n_{i-}+\lambda_{i j} \sqrt{3} T_{i}\right)\left(n_{j-}+\lambda_{i j} \sqrt{3} T_{j}\right)-(3+\eta)\right]
\end{aligned}
$$

where $\lambda_{i j}=(-1)^{\vec{\delta} \vec{y}}$ with $\vec{y}$ being a unit vector in the $b$ direction, and

(ii) for the bonds $\langle i j\rangle \perp(a, b)$, i.e., along the $c$ axis,

$$
\begin{aligned}
\mathcal{H}_{\perp}= & J \sum_{\langle i j\rangle \perp}\left[(4-2 \eta) \vec{S}_{i z z} \cdot \vec{S}_{j z z}-\eta\left(\vec{S}_{i x x} \cdot \vec{S}_{j z z}+\vec{S}_{i z z} \cdot \vec{S}_{j x x}\right)\right. \\
& \left.+(1+2 \eta) n_{i-} n_{j-}-\frac{1}{4}(3+\eta)\right]
\end{aligned}
$$

Here and in the following sections we consider a 3D model with $\beta=1$. We note that the orbital interactions (2.12) are quite different in $H_{\|}$and $H_{\perp}$; propagating spin-and-orbital excitations are possible only within the $(a, b)$ planes, where 
they are coupled to the spin excitations, while in the $c$ direction only pure spin excitations and pure orbital excitations occur, which are decoupled from one another. This breaking of symmetry between $H_{\|}$and $H_{\perp}$ is a consequence of the choice of basis as $|x\rangle$ and $|z\rangle$ orbitals.

In the following sections we consider transverse and longitudinal excitations in the various symmetry-broken states. The transverse excitations, i.e., spin waves and spin-andorbital waves, are calculated using the spin-changing operators which make a transition to a state realized in a classical phase at a given site $i$; for example for the AFxx phase these operators are for $i$ in the A (spin-up) sublattice,

$$
S_{i x x}^{+}=d_{i x \uparrow}^{\dagger} d_{i x \downarrow}, \quad K_{i x z}^{+}=d_{i x \uparrow}^{\dagger} d_{i z \downarrow} .
$$

The longitudinal excitations without spin-flip are most conveniently obtained starting from spin-dependent orbital excitation operators,

$$
T_{i x z \sigma}=d_{i x \sigma}^{\dagger} d_{i z \sigma}, \quad T_{i z x \sigma}=d_{i z \sigma}^{\dagger} d_{i x \sigma} .
$$

The commutation relations for these operators are presented in Appendix B.

\section{B. Antiferromagnetic AFxx phase}

The nature and dispersion of elementary excitations in the spin-orbital model (2.9) can be conveniently studied in the leading order of the $1 / S$ expansion using the Green-function formalism. We note, however, that equivalent results for the AFxx and AFzz phases can be obtained using instead an expansion around a classical saddle point with Schwinger bosons. ${ }^{69}$

We start from the equations of motion for the Green functions generated by the excitation operators (4.10) written in the energy representation ${ }^{70,71}$

$$
\begin{aligned}
& E\left\langle\left\langle S_{i x x}^{+} \mid \cdots\right\rangle\right\rangle=\frac{1}{2 \pi}\left\langle\left[S_{i x x}^{+}, \ldots\right]\right\rangle+\left\langle\left\langle\left[S_{i x x}^{+}, H\right] \mid \cdots\right\rangle\right\rangle, \\
& E\left\langle\left\langle K_{i x z}^{+} \mid \cdots\right\rangle\right\rangle=\frac{1}{2 \pi}\left\langle\left[K_{i x z}^{+}, \ldots\right]\right\rangle+\left\langle\left\langle\left[K_{i x z}^{+}, H\right] \mid \cdots\right\rangle\right\rangle,
\end{aligned}
$$

where the average of the commutator on the right-hand side, e.g., $\left\langle\left[S_{i x x}^{+}, S_{j x x}^{-}\right]\right\rangle$, is evaluated in the classical ground state. The excitation operators were chosen as leading to the local states $|i x \uparrow\rangle$ realized at one of the sublattices in the ground state of the AFxx phase. As usually, the commutators in Eqs. (4.12) and (4.13) generate higher-order Green functions. In contrast to the HAF, it does not suffice to consider the spinflip Green function $\left\langle\left\langle S_{i x x}^{+} \mid \cdots\right\rangle\right\rangle$, as the spin flips may also occur together with an accompanying orbital flip, as described by $\left\langle\left\langle K_{i x z}^{+} \mid \cdots\right\rangle\right\rangle$.

We derived the equations of motion for the Green functions generated by the set of operators $\left\{S_{i x x}^{+}, K_{i x z}^{+}, S_{j x x}^{+}, K_{j x z}^{+}\right\}$, where $i \in A$ and $j \in B$, and used the random-phase approximation (RPA) for spinlike operators which linearizes the equations of motion by a decoupling procedure. $^{70,71}$ Thereby the operators which have nonzero expectation values in the considered classical state give finite contributions, e.g., for the first spin-flip Green function one uses

$$
\left\langle\left\langle S_{i x x}^{+} S_{m x x}^{z} \mid \cdots\right\rangle\right\rangle \simeq\left\langle S_{m x x}^{z}\right\rangle\left\langle\left\langle S_{i x x}^{+} \mid \cdots\right\rangle\right\rangle,
$$

and a similar formula for the mixed spin-and-orbital excitation described by $\left\langle\left\langle K_{i x z}^{+} \mid \cdots\right\rangle\right\rangle$,

$$
\left\langle\left\langle K_{i x z}^{+} S_{m x x}^{z} \mid \cdots\right\rangle\right\rangle \simeq\left\langle S_{m x x}^{z}\right\rangle\left\langle\left\langle K_{i x z}^{+} \mid \cdots\right\rangle\right\rangle .
$$

It is crucial that the decoupled operators have different site indices, and thus the decoupling procedure preserves the local commutation rules given in Appendix B. Instead, if one uses products of spin and orbital operators, e.g., $K_{i x z}^{+}$ $=S_{i x x}^{+} \sigma_{i}^{+}$, one is tempted to decouple these operators locally ${ }^{72,73}$ which would violate the algebraic structure of the so(4) Lie algebra.

In the present case of the AFxx phase one uses the respective Néel state average values,

$$
\begin{gathered}
\left\langle S_{i x x}^{z}\right\rangle=-\left\langle S_{j x x}^{z}\right\rangle=\frac{1}{2}, \\
\left\langle n_{i-}\right\rangle=\left\langle n_{j-}\right\rangle=\frac{1}{2},
\end{gathered}
$$

where $i \in A$ and $j \in B$, and $A$ and $B$ are the two sublattices in a 2D lattice for the AFxx phase. All the remaining averages vanish, as this phase has a pure $|x\rangle$-orbital character at every site, which simplifies significantly the equations of motion which result from the RPA procedure.

The translational invariance of the Néel state implies that the transformed Green functions are diagonal in the reduced Brillouin zone (BZ). As in the HAF, the Fourier transformed functions are defined for the Green functions which describe the spin dynamics on a given sublattice, either $A$ or $B$. For instance, the pure spin-flip Green functions are transformed as follows:

$$
\begin{aligned}
& \left\langle\left\langle S_{\vec{k} x x}^{+} \mid \cdots\right\rangle\right\rangle_{A}=\frac{1}{\sqrt{N}} \sum_{i \in A} e^{i \vec{k} \vec{R}_{i}}\left\langle\left\langle S_{i x x}^{+} \mid \cdots\right\rangle\right\rangle_{A}, \\
& \left\langle\left\langle S_{\vec{k} x x}^{+} \mid \cdots\right\rangle\right\rangle_{B}=\frac{1}{\sqrt{N}} \sum_{j \in B} e^{i \vec{k} \vec{R}_{j}}\left\langle\left\langle S_{j x x}^{+} \mid \cdots\right\rangle\right\rangle_{B},
\end{aligned}
$$

where $N$ is the number of sites in one sublattice. Hence the problem of finding the elementary excitations of the considered spin-orbital model (2.9) reduces to the diagonalization of a $4 \times 4$ dynamical matrix at each $\vec{k}$ point, as given in Appendix C.

The symmetric positive and negative eigenvalues $\pm \omega_{\vec{k}}^{(n)}$, with $n=1,2$, solved from the matrix in Eq. (C2) may be written in the following form for the AFxx phase:

$$
\begin{aligned}
{\left[\omega_{\vec{k}}^{(n)}\right]^{2}=} & J^{2}\left(\lambda_{x}^{2}+\tau_{x}^{2}-Q_{x \vec{k}}^{2}-R_{\vec{k}}^{2}-2 P_{x \vec{k}}^{2}\right) \\
& \pm J^{2}\left[\left(\lambda_{x}^{2}-\tau_{x}^{2}\right)^{2}-2\left(\lambda_{x}^{2}-\tau_{x}^{2}\right)\left(Q_{x \vec{k}}^{2}-R_{\vec{k}}^{2}\right)\right. \\
& -4\left(\lambda_{x}-\tau_{x}\right)^{2} P_{x \vec{k}}^{2}+\left(Q_{x \vec{k}}^{2}+R_{\vec{k}}^{2}+2 P_{x \vec{k}}^{2}\right)^{2} \\
& \left.-4\left(Q_{x \vec{k}} R_{\vec{k}}-P_{x \vec{k}}^{2}\right)^{2}\right]^{1 / 2} .
\end{aligned}
$$


Here the quantities $\lambda_{\alpha}$ and $\tau_{\alpha}$ play the role of local potentials and follow from the model parameters $E_{z}$ and $J_{H}$ :

$$
\begin{gathered}
\lambda_{x}=\frac{9}{2}-3 \eta, \\
\tau_{x}=\frac{7}{2}-4 \eta-2-\eta+\varepsilon_{z} .
\end{gathered}
$$

The remaining terms are $\vec{k}$ dependent, and depend on

$$
\begin{gathered}
\gamma_{+}(\vec{k})=\frac{1}{2}\left(\cos k_{x}+\cos k_{y}\right), \\
\gamma_{-}(\vec{k})=\frac{1}{2}\left(\cos k_{x}-\cos k_{y}\right), \\
\gamma_{z}(\vec{k})=\cos k_{z} .
\end{gathered}
$$

The quantities $Q_{x \vec{k}}$ and $P_{x \vec{k}}$ for the AFxx phase take the form,

$$
\begin{gathered}
Q_{x \vec{k}}=\left(\frac{9}{2}-3 \eta\right) \gamma_{+}(\vec{k}), \\
P_{x \vec{k}}=\frac{1}{2} \sqrt{3}(3-\eta) \gamma_{-}(\vec{k}),
\end{gathered}
$$

while the last dispersive term,

$$
R_{\vec{k}}=\frac{3}{2} \gamma_{+}(\vec{k}),
$$

carries no index and remains identical for both AF phases (AFxx and AFzz). We emphasize that the coupling between the spin-wave and spin-and-orbital-wave excitations occurs due to the terms $\propto P_{x \vec{k}}$, as seen from Eq. (C2). It vanishes in the planes of $k_{x}= \pm k_{y}$, but otherwise plays an important role, as discussed in Sec. V. In the limit of large $E_{z} \rightarrow \infty$, Eq. (4.19) reproduces the spin-wave excitations for a $2 \mathrm{D}$ antiferromagnet with an AF superexchange interaction of $J\left(\frac{9}{4}\right.$ $\left.-\frac{3}{2} \eta\right)$

$$
\omega_{\vec{k}}^{(1)}=J\left(\frac{9}{2}-3 \eta\right)\left[1-\gamma_{+}^{2}(\vec{k})\right]^{1 / 2},
$$

while the dispersion of the high-energy spin-and-orbital excitation, $\omega_{\vec{k}}^{(2)} \simeq E_{z}$, becomes negligible. As explained above, both modes are doubly degenerate.

Consider now the orbital (excitonic) excitations generated by the orbital-flip operators (4.11). They are found by considering the equations of motion,

$$
\begin{array}{r}
E\left\langle\left\langle T_{i \alpha \beta \uparrow} \mid \cdots\right\rangle\right\rangle=\frac{1}{2 \pi}\left\langle\left[T_{i \alpha \beta \uparrow}, \ldots\right]\right\rangle+\left\langle\left\langle\left[T_{i \alpha \beta \uparrow}, H\right] \mid \cdots\right\rangle\right\rangle, \\
E\left\langle\left\langle T_{i \alpha \beta \downarrow} \mid \cdots\right\rangle\right\rangle=\frac{1}{2 \pi}\left\langle\left[T_{i \alpha \beta \downarrow}, \ldots\right]\right\rangle+\left\langle\left\langle\left[T_{i \alpha \beta \downarrow}, H\right] \mid \cdots\right\rangle\right\rangle,
\end{array}
$$

and the commutators are calculated using the rules (B7). In general, one finds four different excitation operators at each site. However, making a Fourier transformations as for the transverse operators (4.18), one may show that only two operators per sublattice suffice to describe the modes in an antiferromagnet. The structure of the respective RPA dynamical matrix is given in Appendix C. The orbital excitations which follow from Eq. (C3) are in general given by

$$
\zeta_{\vec{k}}=J\left[u_{\alpha}\left(u_{\alpha} \pm 2 \rho_{\alpha \vec{k}}\right)\right]^{1 / 2},
$$

(a)

(b)

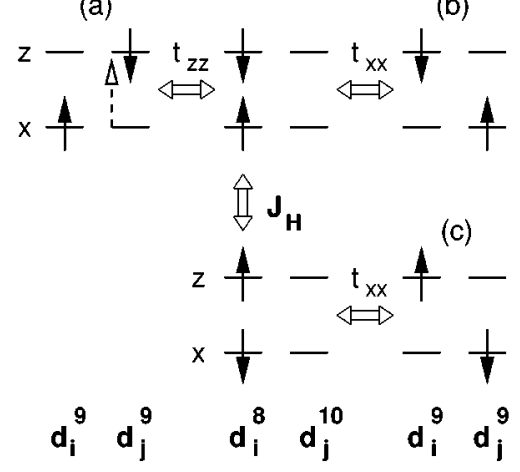

FIG. 8. Schematic propagation of the orbital (excitonic) excitation (a). If $J_{H}=0$, an orbital excitation can propagate only to state (b) and is accompanied by a spin flip (top), while $J_{H}>0$ allows also the spin flip in the intermediate $d_{i}^{8}$ state, and thus the propagation without spin flip (c) becomes possible (bottom).

yielding two, in general nondegenerate, positive-frequency modes. In the AFxx phase one finds

$$
\begin{gathered}
u_{x}=\varepsilon_{z}-3 \eta, \\
\rho_{x \vec{k}}=\frac{3}{2} \eta \gamma_{+}(\vec{k}) .
\end{gathered}
$$

It is important to realize that the propagation of longitudinal excitations, being equivalent to a finite dispersion of longitudinal modes, becomes possible only at $\eta>0$. This follows from the multiplet structure of the excited $d^{8}$ states, which allows a spin-flip between the orbitals in the $\left|{ }^{1} E_{\theta}\right\rangle$ and in the $S^{z}=0$ component of the $\left|{ }^{3} A_{2}\right\rangle$ state only if $J_{H} \neq 0$, as illustrated in Fig. 8. The processes $\sim t_{x z}$ are not included, as they would lead to a final state shown in Fig. 8(b), i.e., to a propagation of a spin-and-orbital excitation which was already considered above. In contrast, the relevant longitudinal orbital excitation in the symmetry-broken state implies that

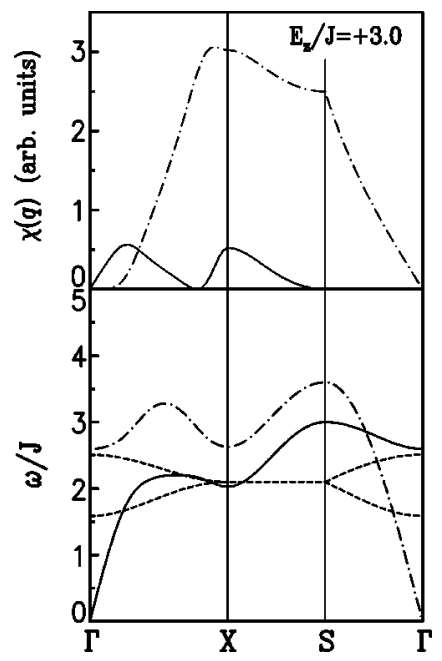

FIG. 9. Lower panel: spin-wave and spin-and-orbital-wave transverse excitations (full line and dashed-dotted line) and longitudinal excitations (dashed lines) in AFxx phase; upper panel: corresponding neutron intensities of the transverse excitations. Parameters: $E_{z} / J=3.0$ and $J_{H} / U=0.3$. 
the exciton has the same spin as imposed by the Néel state of the background; this state is shown in Fig. 8(c). Therefore, in a perfect Néel state without FM interactions due to $\eta \neq 0$, only local orbital excitations are possible. These local excitations cost no energy in the limit of $\varepsilon_{z} \rightarrow 0$ which demonstrates again the frustration of magnetic interactions at the classical degeneracy point, $\varepsilon_{z}=\eta=0$.

An example of the excitation spectra is shown in Fig. 9 for the main directions in the 2D BZ, with $X=(\pi, 0)$ and $S$ $=(\pi / 2, \pi / 2)$. Near the $\Gamma=(0,0)$ point one finds a (doublydegenerate) Goldstone mode $\omega_{\vec{k}}^{(1)}$ with dispersion $\sim k$ at $\vec{k}$ $\rightarrow 0$, as in the HAF, and a second (doubly-degenerate) transverse mode at higher energy, $\omega_{\vec{k}}^{(2)} \simeq \omega_{0}+a k^{2}$. Near $\Gamma$ the Goldstone mode is essentially purely spin wave, the second mode purely spin-and-orbital wave. With increasing $\vec{k}$ these modes start to mix due to the $P_{x \vec{k}}$ term along the $\Gamma-X$ direction. This is best illustrated by the intensity measured in the neutron-scattering experiments, which see only the spinwave component in each transverse mode, as explained in more detail in Appendix D. The intensity $\chi(\vec{q})$ moves from one mode to the other along the $\Gamma-X$ direction in the $2 \mathrm{D}$ BZ (Fig. 9), demonstrating that indeed the lowest (highest) mode is predominantly spin-wave-like (spin-and-orbital-wave-like) before the anticrossing point, while this is reversed after the anticrossing of the two modes. Thus we make here a specific prediction that two spin-wave-like modes could be measurable in certain parts of the $2 \mathrm{D} \mathrm{BZ}$, in particular in the vicinity of an anticrossing, if only an AFxx phase was realized for parameters not too distant from the classical degeneracy point. This provides a possibility of measuring orbital excitations by neutron scattering. Unfortunately, for the realistic parameters for the cuprates, ${ }^{28}$ one finds $E_{z} / J \simeq 10$ which makes the spin-and-orbital excitation and the changes of the spin-wave dispersion hardly visible in neutron spectroscopy.

The orbital (longitudinal) excitations are found for the parameters of Fig. 9 at a finite energy, being of the same order of magnitude as the energy of the spin-and-orbital excitation, $\omega_{\vec{k}}^{(2)}$. The weak dispersion of these modes follows from the spin-flip processes in the excited states, as explained in Fig. 8 and discussed above. We emphasize that the orbital mode has a gap and does not couple to any spin excitation. At the classical degeneracy point $M$ the orbital mode falls to zero energy and is dispersionless, expressing that the orbital can be changed locally without any cost in energy.

\section{Antiferromagnetic AFzz phase}

The transverse excitations in the AFzz phase are determined by considering the complementary set of Green functions to that given in Eqs. (4.12) and (4.12):

$$
E\left\langle\left\langle S_{i z z}^{+} \mid \cdots\right\rangle\right\rangle=\frac{1}{2 \pi}\left\langle\left[S_{i z z}^{+}, \ldots\right]\right\rangle+\left\langle\left\langle\left[S_{i z z}^{+}, H\right] \mid \cdots\right\rangle\right\rangle,
$$

$$
E\left\langle\left\langle K_{i z x}^{+} \mid \cdots\right\rangle\right\rangle=\frac{1}{2 \pi}\left\langle\left[K_{i z x}^{+}, \ldots\right]\right\rangle+\left\langle\left\langle\left[K_{i z x}^{+}, H\right] \mid \cdots\right\rangle\right\rangle,
$$

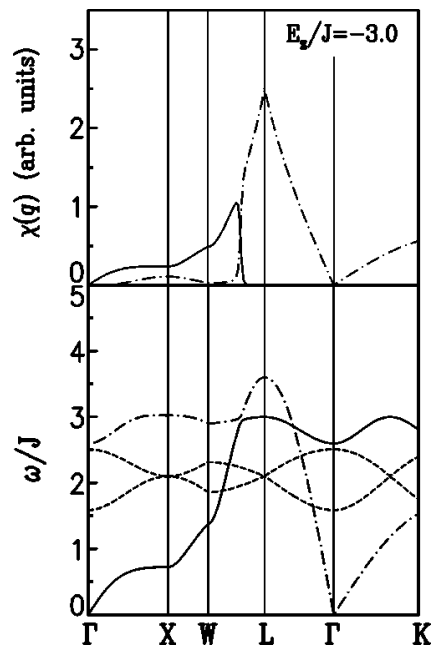

FIG. 10. The same as in Fig. 9, but for the AFzz phase, as obtained for $E_{z} / J=-3.0$ and $J_{H} / U=0.3$.

with the excitations to the local $|i z \uparrow\rangle$ states. As usually, the average of the commutator on the right-hand side is next evaluated in the classical ground state. After obtaining the RPA equations, we thus use the following nonvanishing averages:

$$
\begin{aligned}
& \left\langle S_{i z z}^{z}\right\rangle=-\left\langle S_{j z z}^{z}\right\rangle=\frac{1}{2}, \\
& \left\langle n_{i-}\right\rangle=\left\langle n_{j-}\right\rangle=-\frac{1}{2},
\end{aligned}
$$

in the AFzz phase. This leads again to the general form (C2), with all the elements except for $R_{\vec{k}}$ replaced by,

$$
\begin{gathered}
\lambda_{z}=\frac{1}{2}-\eta+2(2-\eta), \\
\tau_{z}=-\frac{1}{2}-\eta+2(1-2 \eta)-\varepsilon_{z}, \\
Q_{z \vec{k}}=\left(\frac{1}{2}-\eta\right) \gamma_{+}(\vec{k})+2(2-\eta) \gamma_{z}(\vec{k}), \\
P_{z \vec{k}}=\frac{1}{2} \sqrt{3}(1-\eta) \gamma_{-}(\vec{k}) .
\end{gathered}
$$

Thus the transverse excitations have the same form (4.19) as in the AFxx phase, but the above quantities (4.38)-(4.41) have to be used.

In the limit of large $E_{z} \rightarrow-\infty$ one finds the spin wave for a 3D anisotropic antiferromagnet with strong superexchange equal to $2 J(2-\eta)$ along the $c$ axis, and weak superexchange $\frac{1}{4} J(1-2 \eta)$ within the $(a, b)$ planes,

$$
\begin{aligned}
\omega_{\vec{k}}^{(1)}= & J\left\{\left[\left(\frac{1}{2}-\eta\right)+2(2-\eta)\right]^{2}\right. \\
& \left.-\left[\left(\frac{1}{2}-\eta\right) \gamma_{+}(\vec{k})+2(2-\eta) \gamma_{z}\right]^{2}\right\}^{1 / 2},
\end{aligned}
$$

while the spin-and-orbital excitation $\omega_{\vec{k}}^{(2)} \simeq-E_{z}$ is dispersionless. Again, both these transverse modes are doubly degenerate. The orbital excitations in the AFzz phase are found using the equations of motion of the form (4.29) and (4.30) which lead to Eq. (4.31) with

$$
\begin{gathered}
u_{z}=-\varepsilon_{z}-3 \eta, \\
\rho_{z, \vec{k}}=-\frac{3}{2} \eta \gamma_{+}(\vec{k}),
\end{gathered}
$$


and we find again zero-energy nondispersive modes at $\varepsilon_{z}$ $=\eta=0$.

The representative excitation spectrum for the AFzz phase is shown in Fig. 10. We use the 3D BZ for a bcc lattice with the standard notation: $W=(\pi, \pi / 2,0), L=(\pi / 2, \pi / 2, \pi / 2)$, and $K=(3 \pi / 4,3 \pi / 4,0)$. The transverse modes have qualitatively the same behavior as in the $2 \mathrm{D}$ AFxx phase, and one finds a Goldstone mode $\omega_{\vec{k}}^{(1)}$ at the $\Gamma$ point which is spinwave-like, accompanied by a finite energy spin-and-orbital mode $\omega_{\vec{k}}^{(2)}$. The first one is linear, while the second changes quadratically with increasing $\vec{k}$. The dispersion in the $\Gamma-X$ direction is, however, only $\sim 0.7 \mathrm{~J}$, while in the AFxx phase a large dispersion of $\sim 2.5 \mathrm{~J}$ was found (Fig. 9). This demonstrates the very large difference between the superexchange in the $(a, b)$ planes in the two AF phases.

Here one should bear in mind, that in a strongly anisotropic antiferromagnet, such as the AFzz phase, the dispersion of the spin-wave mode in the $\left(k_{x}, k_{y}\right)$ plane is roughly $\left(2 J_{a b} J_{c}\right)^{1 / 2}$, so actually enhanced by $\left(J_{c} / 2 J_{a b}\right)^{1 / 2}$ compared with the planar exchange constant. In fact, there is also strong mixing between spin wave and spin-and-orbital wave along $\Gamma-X$, depressing $\omega_{X}^{(1)}$ at the $X$ point by no less than $0.5 J$ from its pure spin-wave value. The mixing effect is also visible in the relatively large neutron intensity of the second mode. By contrast, the transverse excitations are rather pure all along the $W-L$ direction [where the neutron intensity $\chi(\vec{q})$ is larger], except in the regime where $\omega_{\vec{k}}^{(1)} \simeq \omega_{\vec{k}}^{(2)}$ and the neutron intensity is distributed between the modes. However, owing to the abruptness of the anticrossing, the range where the modes have simultaneously appreciable intensity is very narrow, and their energetic proximity then makes it likely that they would be measured as a single broad maximum.

The (longitudinal) orbital excitation is found at the $X$ and $L$ points at the same energy as that of a local excitation from $|z\rangle$ to $|x\rangle$ orbital (see Fig. 10). It depends only on the energy difference between the orbitals, and has a weak dispersion by the same mechanism as described above for the AFxx phase (Fig. 8).

\section{Mixed-orbital FFA phase}

The excitation operators which couple to the local states in a symmetry-broken phase with mixed orbitals are linear combinations of the operators considered in Secs. IV B and IV C. The classical order is described by four sublattices, $A$ and $B(C$ and $D)$ in even (odd) $(a, b)$ planes, with $C(D)$ sites being the nearest neighbors of $A(B)$ sites. We assume the alternation of orbitals also along the $c$ axis as only this state was found to be stable in the present LSW theory. It is therefore convenient to make a unitary transformation of the Hamiltonian (2.9) to new orbitals defined as follows for $i$ $\in A$ or $i \in D$ sublattice:

$$
\left(\begin{array}{c}
|i \mu\rangle \\
|i \nu\rangle
\end{array}\right)=\left(\begin{array}{cc}
\cos \theta & \sin \theta \\
-\sin \theta & \cos \theta
\end{array}\right)\left(\begin{array}{l}
|i z\rangle \\
|i x\rangle
\end{array}\right),
$$

and for $j \in B$ or $j \in C$ sublattice,

$$
\left(\begin{array}{l}
|j \mu\rangle \\
|j \nu\rangle
\end{array}\right)=\left(\begin{array}{cc}
\cos \theta & -\sin \theta \\
\sin \theta & \cos \theta
\end{array}\right)\left(\begin{array}{l}
|j z\rangle \\
|j x\rangle
\end{array}\right) .
$$

With these definitions and by choosing the angle $\theta$ at the value which minimizes the classical energy (3.11), we guarantee that $|i \mu\rangle$ and $|j \mu\rangle$, respectively, are at each site the orbital state realized in the classical MOFFA phase, which is $G$-type with respect to the orbital ordering, while $|i \nu\rangle$ and $|j \nu\rangle$ are the excited state, so that one can readily define the excitation operators pertinent to the symmetry-broken ground state of this phase. Thus the spin, spin-and-orbital, and orbital operators in terms of the new orbital states $\{|\mu\rangle,|\nu\rangle\}$ defined by Eqs. (4.45) and (4.46) are

$$
\begin{gathered}
\mathcal{K}_{i \alpha \beta}^{+}=|i \alpha \uparrow\rangle\langle i \beta \downarrow|, \\
\mathcal{K}_{i \alpha \beta}^{z}=\frac{1}{2}(|i \alpha \uparrow\rangle\langle i \beta \uparrow|-| i \alpha \downarrow\rangle\langle i \beta \downarrow|), \\
\mathcal{T}_{i-}=\frac{1}{2} \sum_{\sigma}(|i \mu \sigma\rangle\langle i \nu \sigma|+| i \nu \sigma\rangle\langle i \mu \sigma|), \\
\mathcal{N}_{i-}=\frac{1}{2} \sum_{\sigma}(|i \mu \sigma\rangle\langle i \mu \sigma|-| i \nu \sigma\rangle\langle i \nu \sigma|) .
\end{gathered}
$$

The new operators, $\overrightarrow{\mathcal{K}}_{i \alpha \beta}, \mathcal{T}_{i}$ and $\mathcal{N}_{i-}$, fulfill the same commutation rules as the nontransformed operators, $\vec{K}_{i \alpha \beta}, T_{i}$, and $n_{i-}$, respectively; they are given in Appendix B. To simplify the notation we also introduce total spin and spinand-orbital operators,

$$
\begin{aligned}
\overrightarrow{\mathcal{S}}_{i} & =\overrightarrow{\mathcal{S}}_{i \mu \mu}+\overrightarrow{\mathcal{S}}_{i \nu \nu}, \\
\overrightarrow{\mathcal{K}}_{i} & =\overrightarrow{\mathcal{K}}_{i \mu \nu}+\overrightarrow{\mathcal{K}}_{i \nu \mu} .
\end{aligned}
$$

The transverse excitations may be found starting from the relevant raising operators that lead to the local state $|i \mu \uparrow\rangle$ realized in one of the sublattices, analogous to those introduced for the AFxx phase (4.10), i.e., the set $\left\{\mathcal{S}_{i \mu \mu}^{+}, \mathcal{K}_{i \mu \nu}^{+}, \mathcal{S}_{j \mu \mu}^{+}, \mathcal{K}_{j \mu \nu}^{+}, \mathcal{S}_{k \mu \mu}^{+}, \mathcal{K}_{k \mu \nu}^{+}, \mathcal{S}_{l \mu \mu}^{+}, \mathcal{K}_{l \mu \nu}^{+}\right\}$, where $i \in A, j \in B, k \in C$, and $l \in D$; they lead as usual to the orbitals $\{|i \mu\rangle,|j \mu\rangle\}$ (3.4) realized in the MOFFA phase,

$$
\begin{aligned}
& E\left\langle\left\langle\mathcal{S}_{i \mu \mu}^{+} \mid \cdots\right\rangle\right\rangle=\frac{1}{2 \pi}\left\langle\left[\mathcal{S}_{i \mu \mu}^{+}, \ldots\right]\right\rangle+\left\langle\left\langle\left[\mathcal{S}_{i \mu \mu}^{+}, \mathcal{H}\right] \mid \cdots\right\rangle\right\rangle, \\
& E\left\langle\left\langle\mathcal{K}_{i \mu \nu}^{+} \mid \cdots\right\rangle\right\rangle=\frac{1}{2 \pi}\left\langle\left[\mathcal{K}_{i \mu \nu}^{+}, \ldots\right]\right\rangle+\left\langle\left\langle\left[\mathcal{K}_{i \mu \nu}^{+}, \mathcal{H}\right] \mid \cdots\right\rangle\right\rangle,
\end{aligned}
$$

where the rotated Hamiltonian $\mathcal{H}$ given in Appendix $\mathrm{C}$ is obtained by the inverse tranformations to those given by Eqs. (4.45) and (4.46).

The longitudinal excitations can be obtained from operators similar to those used in the AFxx and AFzz phases (4.11),

$$
\mathcal{T}_{i \mu \nu \uparrow}=d_{i \mu \uparrow}^{\dagger} d_{i \nu \uparrow}, \quad \mathcal{T}_{i \nu \mu \uparrow}=d_{i \nu \uparrow}^{\dagger} d_{i \mu \uparrow},
$$

for the $(a, b)$ planes with the $\uparrow$ spins, and the corresponding $\mathcal{T}_{i \mu \nu \downarrow}$ and $\mathcal{T}_{i \nu \mu \downarrow}$ for the $(a, b)$ planes with the $\downarrow$ spins. The commutation operators for these operators are analogous to 


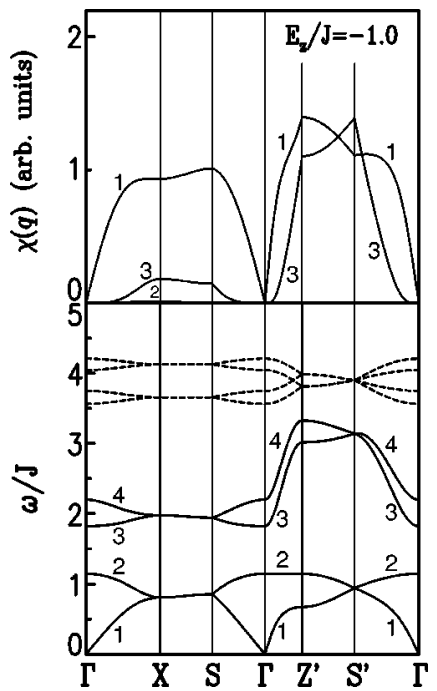

FIG. 11. The same as in Fig. 9, but for the MOFFA phase, as obtained for $E_{z} / J=-1.0$ and $J_{H} / U=0.3$. Different transverse modes are labelled by the increasing indices $i=1, \ldots, 4$ with increasing energy.

those presented in Appendix B and may be easily obtained. The resulting dynamical matrices for both transverse and longitudinal excitations are given in Appendix C; their numerical diagonalization gave the modes presented below. There are four doubly-degenerate positive-frequency transverse modes, and four nondegenerate positive-frequency longitudinal modes, consistent with the MOFFA phase having four sublattices.

An example of the transverse and longitudinal modes in the MOFFA phase is presented in Fig. 11. The modes are shown in the respective BZ which corresponds to the magnetic unit cell of the MOFFA phase: The 2D part along $\Gamma$ $-X-S-\Gamma$ is identical with the AFxx phase (compare Fig. 9), reflecting the orbital alternation, while the AF coupling along the $c$ axis results in the folding of the zone along the $\Gamma-Z \quad$ direction, with $Z^{\prime}=(0,0, \pi / 2)$ and $S^{\prime}$ $=(\pi / 2, \pi / 2, \pi / 2)$. One finds one Goldstone mode, and three other finite-energy modes at the $\Gamma$ point. If no AF coupling along the $c$ axis is present, similar positive-energy modes describe the excitation spectrum in the MOFF phase in the 2D part of the BZ (in the region of stability shown in Fig. 6), and the symmetric negative-frequency modes carry then no weight. In contrast, due to the strong AF interactions in the MOFFA phase, the negative modes give a large energy renormalization due to quantum fluctuations, as discussed in more detail in Sec. V.

The spin-wave and spin-and-orbital-wave excitations are well separated along the $\Gamma-X-S-\Gamma$ path, with a gap of $\sim 0.5 J$, as the FM interactions $\propto J \eta$ are considerably weaker than the orbital interactions which are $\propto J$. Therefore the neutron intensity $\chi(\vec{q})$ is found mainly as originating from the lowest energy mode $\omega_{\vec{k}}^{(1)}$, with a small admixture of the higher-energy spin-and-orbital excitation $\omega_{\vec{k}}^{(3)}$. The magnetic interactions are considerably stronger along the $c$ axis; the modes mix and the higher-energy excitations, $\omega_{\vec{k}}^{(n)}$ with $n=3,4$, have a larger dispersion in the remaining directions with $k_{z} \neq 0$. Strong mixing of the modes in this part of the
$\mathrm{BZ}$ is also visible in the intensity distribution, with the modes $n=1$ and $n=3$ contributing with comparable intensities (Fig. 11). The fact that modes labeled as 2 and 4 have zero intensity is due to the path $\Gamma-Z^{\prime}-S^{\prime}-\Gamma$ being in the high-symmetry $\mathrm{BZ}$ plane where $k_{x}=k_{y}$ so that $\gamma_{-}(\vec{k})=0$. Then modes 2 and 4 have equal amplitude but are exactly out-of-phase between $A$ and $B$ sites as well as between $C$ and $D$ sites, and so their neutron intensities vanish, and only the companion in-phase modes 1 and 3 are observable by neutrons. Unfortunately, no experimental verification of these spectra is possible at present, as the spin excitations measured in neutron scattering for $\mathrm{KCuF}_{3}$ are consistent with the Bethe ansatz and thus suggest a spin-liquid ground state with strong 1D AF correlations instead of the A-AF phase with magnetic LRO. ${ }^{74}$

Interestingly, although the order in the $(a, b)$ planes is FM, the energy of the Goldstone mode increases linearly in all three directions with increasing $\vec{k}$, and the slopes are proportional to the respective exchange interactions. This behavior is a manifestation of the A-AF spin order; a qualitatively similar spectrum is found experimentally in $\mathrm{LaMnO}_{3},{ }^{75}$ where, however, the excitation spectra describe large spins $S=2$ of $\mathrm{Mn}^{3+}$ ions. The rather small dispersion of the spin-wave part at low energies is due to small values of the exchange constants for the actual optimal orientation of orbitals found at $J_{H} / U=0.3$. We note, however, that the $\mathrm{AF}$ interactions along the $c$ axis are much stronger at $J_{H}$ $\rightarrow 0$ than in the present case. The AF structure along the $c$ axis may be easily recognized from the spin-wave mode in the $\Gamma-Z$ direction symmetric with respect to $Z^{\prime}$ $=(0,0, \pi / 2)$, while this mode increases all the way from the $\Gamma$ to the $X$ point. The fact that only two modes have nonzero neutron scattering intensity along $\Gamma-Z^{\prime}-S^{\prime}-\Gamma$ is due to this BZ path being in the high-symmetry BZ plane, where $k_{x}=k_{y}$ and $\gamma_{-}(\vec{k})=0$. Then two modes have equal amplitude but are exactly out-of-phase between $A$ and $B$ sites as well as between $C$ and $D$ sites, and so their neutron intensities vanish, while only the companion in-phase modes are visible to neutrons. Unlike in the AF phases, the purely orbital excitation is here energetically separated from the spinwave and spin-and-orbital-wave modes. The dispersion is quite small and decreases with $\eta$.

\section{E. Mixed-orbital AFF phase}

The elementary excitations in the MOAFF phase may be obtained using a similar scheme to that used in Sec. IV D for the MOFFA phase. First of all, one defines new quantum states which correspond to the minimum of the classical problem. This is realized by a unitary transformation of the Hamiltonian to the new orbitals defined for $i \in A$ sublattice as

$$
\left(\begin{array}{c}
\left|i \mu_{+}\right\rangle \\
\left|i \nu_{+}\right\rangle
\end{array}\right)=\left(\begin{array}{cc}
\cos \theta_{+} & \sin \theta_{+} \\
-\sin \theta_{+} & \cos \theta_{+}
\end{array}\right)\left(\begin{array}{l}
|i z\rangle \\
|i x\rangle
\end{array}\right)
$$

and for $j \in B$ sublattice as

$$
\left(\begin{array}{l}
\left|j \mu_{-}\right\rangle \\
\left|j \nu_{-}\right\rangle
\end{array}\right)=\left(\begin{array}{cc}
\cos \theta_{-} & -\sin \theta_{-} \\
\sin \theta_{-} & \cos \theta_{-}
\end{array}\right)\left(\begin{array}{l}
|j z\rangle \\
|j x\rangle
\end{array}\right)
$$




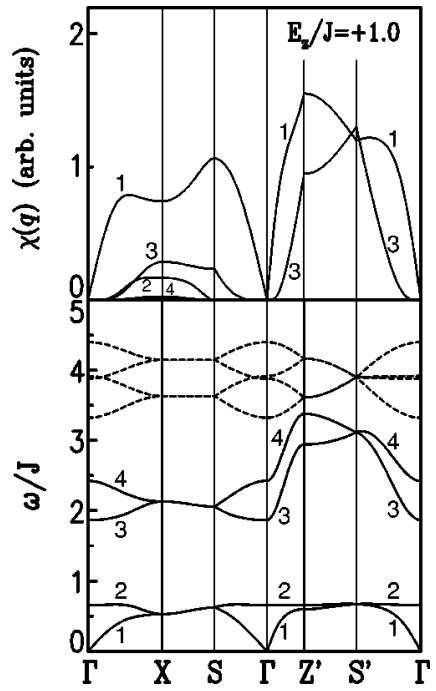

FIG. 12. The same as in Fig. 9, but for the MOAFF phase, as obtained for $E_{z} / J=1.0$ and $J_{H} / U=0.3$.

By choosing the angles $\theta_{+}$and $\theta_{-}$at the values which minimize the classical energy, given by Eqs. (3.14) and (3.15), we guarantee that $\left|i \mu_{+}\right\rangle$and $\left|j \mu_{-}\right\rangle$, respectively, are at each site the orbital state realized in the classical MOFFA phase, and one may easily define the new excitation operators with respect to the symmetry breaking which occurs in this phase; they are analogous to those given in Eqs. (4.47)-(4.52). Next, the Hamiltonian is rotated to the new representation as described in Sec. IV D. We do not present an explicit form of the spin-orbital Hamiltonian (2.9) in this case, as it may be obtained from Eqs. (C4) $-(\mathrm{C} 6)$ by replacing the angle $\theta$ by $\theta_{+}$and $\theta_{-}$for the sublattice $A$ and $B$, respectively. Furthermore, due to the degeneracy between the MOAFF and MOFAF phases, we had to average the crystal field between the two sublattices in the actual calculation.

We have verified that the transverse excitations have a similar dependence on the $\vec{k}$ vector to those found in the MOFFA phase, and we show the representative data in Fig. 12. For convenience, we have rotated the $\mathrm{BZ}$ and use just the same notation as in Fig. 11. The value of the crystal field $E_{z}$ is in the present case effectively smaller by a factor of 2 in comparison with the MOFFA phase. This asymmetry is a consequence of the choice of $|x\rangle$ and $|z\rangle$ states as the orbital basis.

One finds again that the spin-wave and spin-and-orbitalwave excitations are well separated along the $\Gamma-X-S-\Gamma$ path, and the gap between them has increased to $\sim 1.2 \mathrm{~J}$. We note a stronger renormalization of the low-energy modes which follows from weakened FM interactions between the alternating orbitals in the $(b, c)$ planes in the present case as compared with those within the $(a, b)$ planes in the MOFFA phase. Although the orbital excitations are still well separated from the remaining transverse modes, their dispersion is larger than that in Fig. 11.

\section{QUANTUM FLUCTUATIONS}

The size of quantum fluctuation corrections to the classical order parameters determines the stability of the classical phases. As mentioned in Sec. I, frustration of magnetic in- teractions leads in spin models to divergent quantum corrections within the LSW theory. Before calculating these corrections in the present situation, a generalization of the usual RPA procedure to a system with several excitations is necessary. Here we present only the relations needed to calculate the quantum corrections to the LRO parameter and groundstate energy, while more details will be reported separately. ${ }^{76}$

For that purpose, let us denote here the local operators constituting the so(4) Lie algebra at site $i$ as Hubbard operators, $X_{i}^{\alpha \beta}=|i \alpha\rangle\langle i \beta|$. Using the unity operator, $\Sigma_{\beta} X_{i}^{\beta \beta}=1$, the diagonal operator that refers to the state $|i \alpha\rangle$ realized at site $i$ in the classical ground state under consideration may be expanded in terms of the excitation operators

$$
X_{i}^{\alpha \alpha}=1-\sum_{\beta \neq \alpha} X_{i}^{\beta \alpha} X_{i}^{\alpha \beta},
$$

while the diagonal operators referring to an excited state $|i \beta\rangle$ are expressed as

$$
X_{i}^{\beta \beta}=X_{i}^{\beta \alpha} X_{i}^{\alpha \beta} .
$$

Applying these equations to the $z$ th spin component $S_{i}^{z}$ $=S_{i x x}^{z}+S_{i z z}^{z}$ of the total spin at site $i$ in one of the AF phases with pure orbital character (say AFxx for definiteness), one finds, for $i$ in the spin-up sublattice, ${ }^{77}$

$$
\begin{aligned}
& S_{i}^{z}=\frac{1}{2}\left(X_{i}^{x \uparrow, x \uparrow}-X_{i}^{x \downarrow, x \downarrow}+X_{i}^{z \uparrow, z \uparrow}-X_{i}^{z \downarrow, z \downarrow}\right) \\
& =\frac{1}{2} \rrbracket-X_{i}^{x \downarrow, x \uparrow} X_{i}^{x \uparrow, x \downarrow}-X_{i}^{z \downarrow, x \uparrow} X_{i}^{x \uparrow, z \downarrow} \\
& =\frac{1}{2} 1-S_{i x x}^{-} S_{i x x}^{+}-K_{i z x}^{-} K_{i x z}^{+} \text {. }
\end{aligned}
$$

Taking the average one obtains, with the MF value $\left\langle S_{i}^{z}\right\rangle$ $=\frac{1}{2}$,

$$
\begin{aligned}
\left\langle S_{i}^{z}\right\rangle_{\mathrm{RPA}} & =\frac{1}{2}-\left\langle S_{i x x}^{-} S_{i x x}^{+}\right\rangle-\left\langle K_{i z x}^{-} K_{i x z}^{+}\right\rangle \\
& =\frac{1}{2}-\left\langle S_{i}^{-} S_{i}^{+}\right\rangle-\left\langle K_{i}^{-} K_{i}^{+}\right\rangle \\
& =\left\langle S_{i}^{z}\right\rangle-\delta\left\langle S_{i}^{z}\right\rangle,
\end{aligned}
$$

where the second equality is valid because averages like $\left\langle S_{i x x}^{-} S_{i z z}^{+}\right\rangle$are zero since they involve "ghost" modes, so that one may formally replace $S_{i x x}^{+}$by $S_{i x x}^{+}+S_{i z z}^{+}=S_{i}^{+}$, etc. The first contribution $\propto\left\langle S_{i}^{-} S_{i}^{+}\right\rangle$is the usual renormalization due to spin waves, while the second term $\propto\left\langle K_{i}^{-} K_{i}^{+}\right\rangle$stands for the reduction of $\left\langle S_{i}^{z}\right\rangle_{\mathrm{RPA}}$ due to spin-and-orbital-wave excitations. Both terms involve a local excitation preceded by a deexcitation which reproduces the initial local state. As expected only the transverse excitations contribute to the spin renormalization. Note that, since Eq. (5.3) is an exact operator relation, the present procedure guarantees that Eq. (5.4) is a conserving approximation which respects the sum rule for the occupancies of all states, $\Sigma_{\beta}\left\langle X_{i}^{\beta \beta}\right\rangle=1$. The generalization of Eq. (5.4) to the MO phases using the operators (4.47) and (4.48), or to other order parameters, like the orbital polarization, is straightforward.

The local correlation functions which renormalize the order parameter in Eq. (5.1) are determined in the standard way, ${ }^{71}$ 


$$
\left\langle B_{i}^{\dagger} A_{i}\right\rangle=\frac{1}{N} \sum_{\vec{k}} \int_{-\infty}^{+\infty} d \omega \mathcal{A}_{A B^{\dagger}}(\vec{k}, \omega) \frac{1}{\exp (\beta \omega)-1},
$$

where $\beta=1 / k_{B} T$, and

$$
\begin{aligned}
\mathcal{A}_{A B^{\dagger}}(\vec{k}, \omega<0) & =2 \operatorname{Im}\left\langle\left\langle A_{\vec{k}} \mid B_{\vec{k}}^{\dagger}\right\rangle\right\rangle_{\omega-i \epsilon} \\
& =\sum_{\nu<0} \mathcal{A}_{A B^{\dagger}}^{(\nu)}(\vec{k}) \delta\left(\omega-\omega_{\vec{k}}^{(\nu)}\right)
\end{aligned}
$$

is the respective spectral density for the negative frequencies $(\nu<0)$, and $\mathcal{A}_{A B^{\dagger}}^{(\nu)}(\vec{k})$ are the respective spectral weights. Therefore the correlation functions at $T=0$ are found by summing up the total spectral weight at the negative frequencies,

$$
\left\langle B_{i}^{\dagger} A_{i}\right\rangle=\frac{1}{N} \sum_{\vec{k}} \sum_{\nu<0} \mathcal{A}_{A B^{\dagger}}^{(\nu)}(\vec{k}) .
$$

As we show elsewhere ${ }^{76}$ the Hamiltonian of the spinorbital model (2.9) may be expanded in RPA in terms of the excitation and deexcitation operators,

$$
\mathcal{H} \simeq \mathcal{H}_{\mathrm{MF}}+\mathcal{H}_{\mathrm{RPA}}
$$

where $\mathcal{H}_{\mathrm{MF}}$ is given by Eq. (3.1), and

$$
\begin{aligned}
\mathcal{H}_{\mathrm{RPA}}= & \sum_{i \in A} \sum_{\mu \mu^{\prime}} X_{i}^{\mu \alpha} a_{A}^{\mu \mu^{\prime}} X_{i}^{\alpha \mu^{\prime}}+\sum_{j \in B} \sum_{\nu \nu^{\prime}} X_{i}^{\nu \beta} a_{B}^{\nu \nu^{\prime}} X_{i}^{\beta \nu^{\prime}} \\
& +\sum_{\langle i j\rangle} \sum_{\mu \nu}\left(X_{i}^{\mu \alpha} b_{i j}^{\mu \nu} X_{j}^{\beta \nu}+X_{i}^{\alpha \mu} b_{i j}^{\mu \nu} X_{j}^{\nu \beta}\right) \\
& +\sum_{\langle i j\rangle} \sum_{\mu \nu}\left(X_{i}^{\alpha \mu} c_{i j}^{\mu \nu} X_{j}^{\beta \nu}+X_{i}^{\mu \alpha} c_{i j}^{\mu \nu} X_{j}^{\nu \beta}\right)
\end{aligned}
$$

for a two-sublattice phase (the generalization to the foursublattice MO phases is straightforward). The MF part describes the classical problem which was discussed in Sec. III. The RPA part (5.9) describes the many-body problem in a linear approximation, with the fixed indices $\alpha$ and $\beta$ referring to the symmetry-broken state at site $i$ and $j$, respectively. This expansion leads, after changing the order of excitation operators $X_{i}^{\alpha \beta}$ to normal order, and after making straightforward transformations, to a compact expression for the average energy contribution per site,

$$
\begin{aligned}
E_{\mathrm{RPA}} & =\frac{1}{N}\left\langle\mathcal{H}_{\mathrm{RPA}}\right\rangle \\
& =\frac{1}{4}\left[-\operatorname{Tr}\{A\}+\sum_{\nu>0} \frac{2}{N} \sum_{\vec{k}} \omega_{\vec{k}}^{(\nu)}\right],
\end{aligned}
$$

where $A$ is the matrix of positive on-site coefficients $a_{A}^{\mu \mu^{\prime}}, a_{B}^{\nu \nu^{\prime}}$, appearing in the first line of Eq. (5.9), and with the sum running over all modes with positive frequencies (counting doubly-degenerate modes twice) in the reduced BZ. This expression is seen to be a direct generalization of the familiar result for the HAF, the distinction being that more modes contribute here, and so Eq. (5.10) represents the energy gain $\left(E_{\mathrm{RPA}}<0\right)$ due to the reduction in zero-point
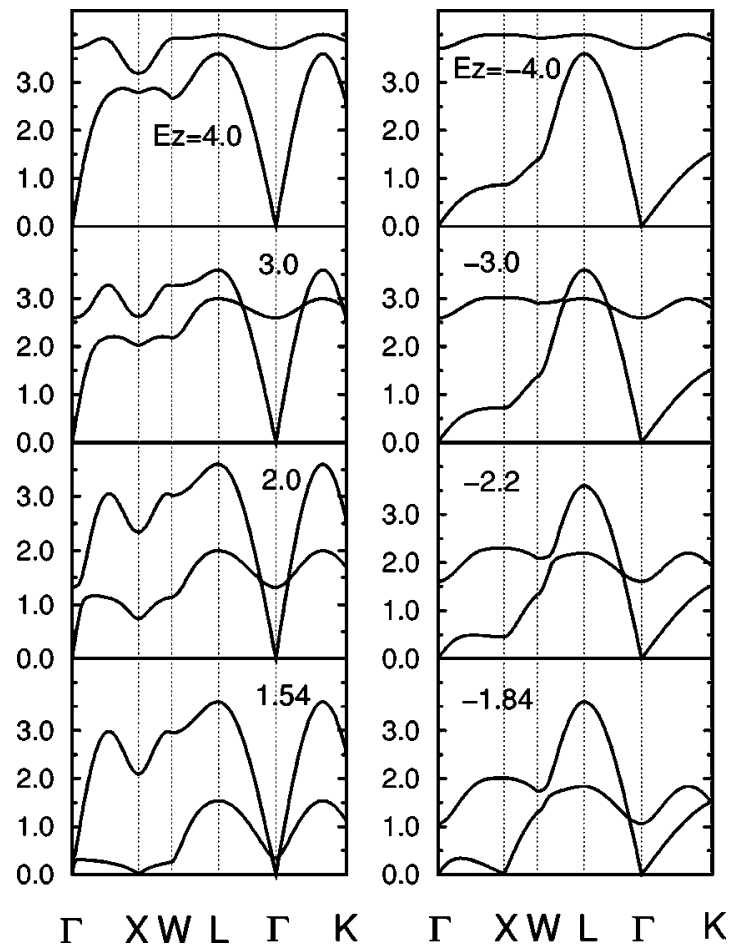

FIG. 13. Spin-wave and spin-and-orbital-wave excitations in the G-AF phases: AFxx (left) and AFzz (right), in the main directions of the 3D BZ for a few values of $E_{z}$ (in the units of $J$ ), and for $J_{H} / U=0.3$. The lower-energy mode becomes soft for $E_{z} / J$ $<1.54\left(E_{z} / J>-1.84\right)$ in the AFxx (AFzz) phase.

energy of the propagating modes in comparison with that of the local excitations. We use Eq. (5.10) to calculate the total energy in RPA,

$$
E=E_{\mathrm{MF}}+E_{\mathrm{RPA}} .
$$

Before discussing the renormalization of the order parameter and the corresponding energies in RPA, we concentrate ourselves on the behavior of the transverse excitations when the crossover lines between the classical phases are approached. As already emphasized in Sec. IV, the spin-wave and spin-and-orbital-wave excitations couple. As a consequence, the modes in all considered phases soften when the transition lines between different classical phases, or classical degeneracy point $M$ are approached. To be more precise, we have verified that the modes soften only after the classical first-order transition lines are crossed, and thus the classical phases remain stable in the region of their existence, while outside they are soon destabilized.

The mode softening is shown for a representative value of $J_{H} / U=0.3$ in Fig. 13 for the two AF phases. ${ }^{78}$ In the AFxx phase the energy scales of both excitations are separated for $E_{z}>4 J$, while the spin-and-orbital mode moves towards zero energy with decreasing $E_{z}$, and finally becomes soft along the $X-R$ direction [with $R=(\pi, \pi, \pi)$ ], i.e., for $\vec{k}$ $=\left(\pi, 0, k_{z}\right)$ and along equivalent lines in the $\mathrm{BZ}$ for $E_{z}$ $\simeq 1.54 \mathrm{~J}$. A similar mode softening is found for the AFzz phase at $E_{z}<0$, with the soft mode along $\Gamma-X$ and equivalent directions in the $\mathrm{BZ}$ at $E_{z} \simeq-1.84 \mathrm{~J}$. This peculiar softening along lines and not at points in the BZ shows that the modes behave 2D like instead of 3D like: ${ }^{78}$ constant- 


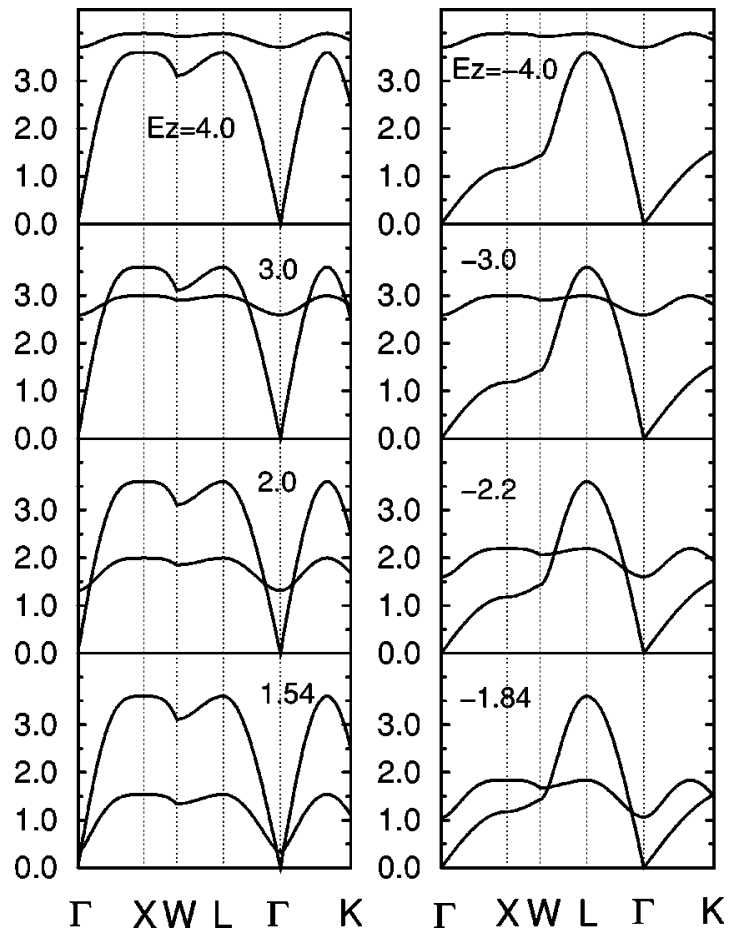

FIG. 14. The same as in Fig. 13, but without the coupling between the spin-wave and spin-and-orbital-wave excitations in both G-AF phases: AFxx (left) and AFzz (right).

frequency surfaces are cylinders contracting towards lines, not spheres contracting towards a point.

By making an expansion of Eq. (4.19) around the softmode lines, one finds that the situation is somewhat different for AFxx and AFzz phase. ${ }^{79}$ In the AFxx phase the lowenergy mode collapses to zero with a quadratic energy dependence on $k_{x}$ and $k_{y}$ (here $\bar{k}_{x}=k_{x}-\pi$ ),

$$
\omega_{\mathrm{AFXX}}(\vec{k}) \rightarrow \Delta_{x}+B_{x}\left(\bar{k}_{x}^{4}+14 \bar{k}_{x}^{2} k_{y}^{2}+k_{y}^{4}\right)^{1 / 2} .
$$

As $\Delta_{x} \rightarrow 0$ at the softening point, this implies finite masses in the perpendicular directions to the lines where $\omega_{\mathrm{AFxx}}(\vec{k})=0$ independently of $k_{z}$. For this reason, quantum fluctuation corrections to the order parameter diverge logarithmically, $\langle\delta S\rangle \sim \int d^{3} k / \omega(\vec{k}) \sim \int d^{2} k /\left(\Delta_{i}+B_{i} k^{2}\right) \sim \ln \Delta_{x}$. As an example, we give explicit expressions at $\eta=0$ :

$$
\Delta_{x}=\frac{9}{2} \frac{\varepsilon_{z}}{\varepsilon_{z}+3}, \quad B_{x}=\frac{27}{16} \frac{1}{\varepsilon_{z}+3},
$$

where one finds that the gap $\Delta_{x} \rightarrow 0$ when $\varepsilon_{z} \rightarrow 0$, i.e., upon approaching the $M=\left(E_{z}, J_{H}\right)=(0,0)$ point at which the $|x\rangle$ orbitals are replaced by $|z\rangle$ orbitals and the classical state changes to the AFzz phase.

A similar expansion in the AFzz phase along the $\Gamma-X$ direction gives instead (again at $\eta=0$ ),

$$
\omega_{\mathrm{AFZZ}}^{2}(\vec{k}) \rightarrow \Delta_{z}^{2}+B_{z}\left(k_{y}^{2}+4 k_{z}^{2}\right),
$$

independently of $k_{x}$, and similarly along the $\Gamma-Y$ direction with $k_{y}$ replaced by $k_{x}$. Although the result for $\omega_{\mathrm{AFZZ}}(\vec{k})$ is similar to that of Eq. (5.12) as long as $\Delta_{z} \neq 0$, the spectrum collapses to a linear $k$ dependence at the point of mode softening. Thus one does not find here a quadratic dependence

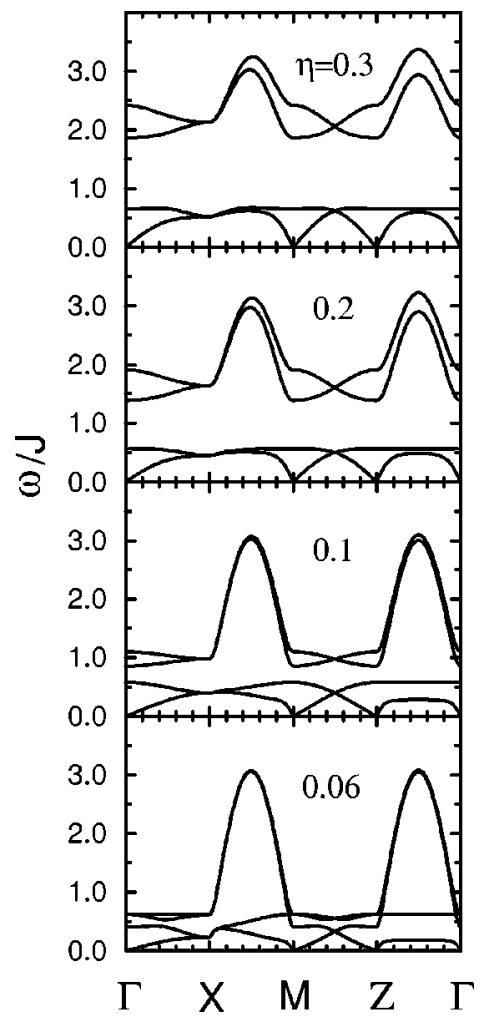

FIG. 15. Spin-wave and spin-and-orbital-wave excitations in MOFFA phase in the main directions of the 3D BZ for a few values of $J_{H} / U$, and for $E_{z} / J=-0.5$. The lower-energy mode becomes soft for $J_{H} / U<0.06$.

with a finite mass as discussed above, but, nevertheless, the quantum correction to the order parameter becomes very large at the softening point and its numerical dependence on the value of $E_{z}$ resembles a diverging quantum correction.

We emphasize that the quasi-2D nature of the dispersion is essential for the occurrence of the diverging quantum corrections in the AFxx and AFzz phases. It enables a 3D system to destabilize LRO by what are essentially 2D fluctuations. So the divergence of the order parameter near the crossover lines in the phase diagram and the associated instability of the classical phases may be regarded as another manifestation of the effective reduction of the dimensionality occurring in the spin-orbital model. We do not present explicitly the softening of the longitudinal modes which also happens at the transition lines but is of minor importance for the stability of AFxx and AFzz phases.

A seemingly attractive way to simplify the calculation of the transverse excitations would be to make a decoupling of the spin waves and spin-and-orbital waves. However, this is equivalent to violating the commutation rules between the spin and spin-and-orbital operators in Appendix B, ${ }^{65}$ and this changes the physics. It gives the same excitation energies as Eq. (4.19), but with $P_{\alpha \vec{k}}=0$; the numerical result is given in Fig. 14. Of course, the spin-wave excitation does not depend then on the orbital splitting $E_{z}$, and the spin-and-orbitalwave excitation gradually approaches the line $\omega_{\vec{k}}=0$ with decreasing $\left|E_{z}\right|$. It has a weak dispersion which depends on $J_{H}$ and on the value of $\left|E_{z}\right|$, and gives an instability at the $\Gamma$ point only, not at lines in the BZ, and in the phase diagram well beyond the transition lines of Fig. 4, i.e., within the 

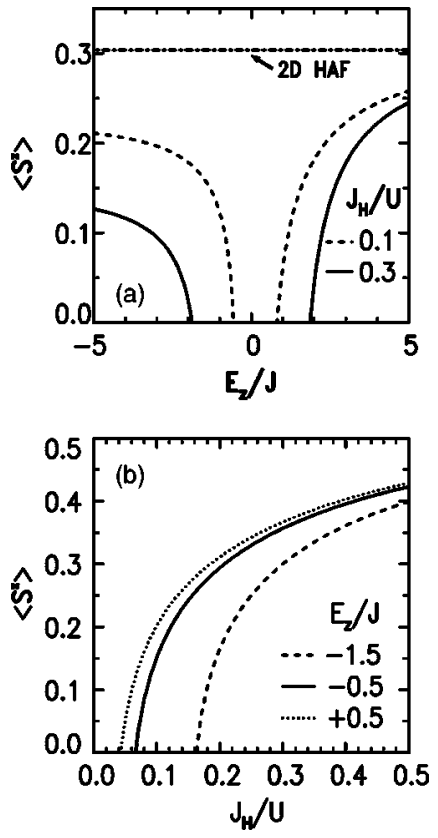

FIG. 16. Renormalization of the magnetic LRO parameter $\left\langle S_{i}^{z}\right\rangle$ by quantum fluctuations as obtained in RPA in: (a) AFzz (left) and AFxx (right) phases as functions of $E_{z} / J$ for $J_{H} / U=0.1$ and 0.3 ; (b) MOFFA phase as functions of $J_{H} / U$ for $E_{z} / J=0.5,-0.5$ and -1.5 .
MOFFA and MOAFF phase for $E_{z}<0$ and $E_{z}>0$, respectively. Such spin-wave and spin-and-orbital-wave modes give, of course, much smaller quantum corrections of the order parameter and energy than the correct RPA spectra of Fig. $13 .^{65}$

The spin-waves in the MOFFA phase, stable at $E_{z}<0$, soften with decreasing $\eta(2.7)$, as shown in Fig. 15. At large $\eta$ the spin-and-orbital waves at high energies are well separated from the spin-wave modes. The latter have a rather small dispersion at $J_{H} / U=0.3$ which follows from relatively weak FM interactions in the $(a, b)$ planes, and AF interactions along the $c$ axis. The modes start to mix stronger with decreasing $\eta$, and finally the gap in the spectrum closes below $\eta=0.1$. The mode softening occurs again along lines in the BZ, namely along the $\Gamma-X$ direction. Unfortunately, we could not perform an analogous analytic expansion of the energies near the softening point to that in the AFxx and AFzz phases, but the numerical results reported here suggest a qualitatively similar behavior to these two phases. The MOAFF phase gives an analogous instability at $E_{z}>0$.

The soft modes in the excitation spectra give a very strong renormalization of the order parameter $\left\langle S^{z}\right\rangle_{\mathrm{RPA}}$ in RPA (5.4) near the mode softening, as shown in Fig. 16. The quantum corrections exceed the MF values of the order parameter in the AFxx and AFzz phases in a region which separates these two types of LRO. Although one might expect that another classical phase with mixed orbitals and FM planes sets in instead, and the actual instabilities where $\delta\left\langle S_{z}\right\rangle \rightarrow \infty$ are

TABLE I. Individual contributions to quantum corrections $\left\langle\delta S^{z}\right\rangle$ of the AF order parameter in $\mathrm{AFxx}$ $\left(E_{z}>0\right)$ and AFzz $\left(E_{z}<0\right)$ phases due to spin wave $\left(\left\langle S^{-} S^{+}\right\rangle\right)$, spin-and-orbital wave $\left(\left\langle K^{-} K^{+}\right\rangle\right)$, and the leading contribution from low-energy mode, $\left\langle\delta S^{z}\right\rangle_{1}$. The values of the magnetic order parameter in RPA are given by $\left\langle S^{z}\right\rangle_{\mathrm{RPA}}$.

\begin{tabular}{crrrrrr}
\hline \hline$J_{H} / U$ & $E_{z} / J$ & $\left\langle S^{-} S^{+}\right\rangle$ & $\left\langle K^{-} K^{+}\right\rangle$ & $\left\langle\delta S^{z}\right\rangle_{1}$ & $\left\langle\delta S^{z}\right\rangle$ & $\left\langle S^{z}\right\rangle_{\mathrm{RPA}}$ \\
\hline 0.0 & -3.0 & 0.2680 & 0.0117 & 0.2731 & 0.2797 & 0.2203 \\
0.0 & -2.0 & 0.2733 & 0.0187 & 0.2606 & 0.2920 & 0.2080 \\
0.0 & -1.0 & 0.2839 & 0.0368 & 0.2146 & 0.3207 & 0.1793 \\
0.0 & 1.0 & 0.2645 & 0.0901 & 0.2440 & 0.3546 & 0.1454 \\
0.0 & 2.0 & 0.2416 & 0.0516 & 0.2426 & 0.2932 & 0.2068 \\
0.0 & 3.0 & 0.2298 & 0.0352 & 0.2455 & 0.2650 & 0.2350 \\
0.1 & -3.0 & 0.2919 & 0.0140 & 0.2963 & 0.3059 & 0.1941 \\
0.1 & -2.0 & 0.2995 & 0.0245 & 0.2757 & 0.3240 & 0.1760 \\
0.1 & -1.0 & 0.3188 & 0.0612 & 0.2339 & 0.3800 & 0.1200 \\
0.1 & 1.0 & 0.2925 & 0.1461 & 0.2864 & 0.4387 & 0.0613 \\
0.1 & 2.0 & 0.2519 & 0.0665 & 0.2493 & 0.3183 & 0.1817 \\
0.1 & 3.0 & 0.2352 & 0.0421 & 0.2519 & 0.2773 & 0.2227 \\
0.2 & -3.0 & 0.3270 & 0.0174 & 0.3291 & 0.3445 & 0.1555 \\
0.2 & -2.0 & 0.3398 & 0.0351 & 0.3023 & 0.3750 & 0.1250 \\
0.2 & 2.0 & 0.2687 & 0.0928 & 0.2647 & 0.3615 & 0.1385 \\
0.2 & 3.0 & 0.2428 & 0.0521 & 0.2593 & 0.2950 & 0.2050 \\
0.2 & 10.0 & 0.2071 & 0.0092 & 0.2077 & 0.2163 & 0.2837 \\
0.3 & -3.0 & 0.3861 & 0.0232 & 0.3834 & 0.4093 & 0.0907 \\
0.3 & -2.0 & 0.4215 & 0.0601 & 0.3720 & 0.4816 & 0.0184 \\
0.3 & 2.0 & 0.3026 & 0.1530 & 0.3179 & 0.4556 & 0.0444 \\
0.3 & 3.0 & 0.2545 & 0.0680 & 0.2706 & 0.3224 & 0.1776 \\
0.3 & 10.0 & 0.2076 & 0.0097 & 0.2083 & 0.2173 & 0.2827 \\
\hline \hline
\end{tabular}




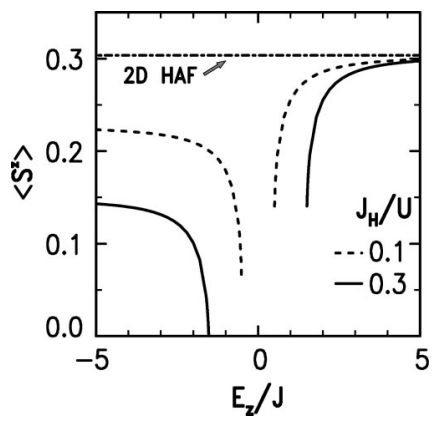

FIG. 17. Renormalization of the magnetic LRO parameter $\left\langle S_{i}^{z}\right\rangle$ by quantum fluctuations obtained for the G-AF phases as in Fig. 16(a), but for decoupled spin-wave and spin-and-orbital-wave excitations shown in Fig. 14.

found indeed beyond the transition lines to another phase, the lines where $\delta\left\langle S^{z}\right\rangle=\left\langle S^{z}\right\rangle$ occur still before the phase boundaries in the phase diagram of Fig. 4 (see Fig. 1 of Ref. 6). This leaves a window where no classical order is stable in between the G-AF and A-AF spin structures.

The origin of such a strong renormalization of $\left\langle S^{z}\right\rangle$ may be better understood by decomposing the quantum corrections into individual contributions as given in Eq. (5.4) (see Table I). The leading correction comes from the local spin fluctuation expressed by $\left\langle S_{i}^{-} S_{i}^{+}\right\rangle$and enhanced with respect to the the pure spin model (HAF), while the spin-and-orbital fluctuation $\left\langle K_{i}^{-} K_{i}^{+}\right\rangle$increases rapidly when the instability lines $\left\langle S^{z}\right\rangle_{\mathrm{RPA}}=0$ are approached. Interestingly, the latter fluctuation is stronger in the AFxx than in the AFzz phase for the same values of $J_{H}$ and $\left|E_{z}\right|$ which demonstrates that the AFzz phase is more robust due to the directionality of the $|z\rangle$ orbitals and the strong AF bonds along the $c$ axis. This asymmetry is also visible in Fig. 16, where $\left\langle S^{z}\right\rangle_{\mathrm{RPA}}$ decreases somewhat faster towards zero for $E_{z}>0$.

In both G-AF phases (AFxx and AFzz) the leading contribution to the renormalization of $\left\langle S^{z}\right\rangle_{\mathrm{RPA}}$ comes from the lower-energy mode, especially at larger values of $J_{H}$. In the

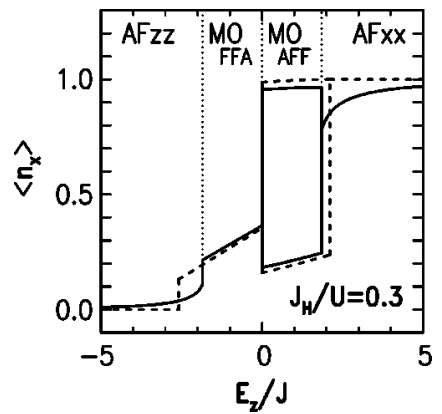

FIG. 18. Average density of $|x\rangle$ holes $\left\langle n_{x}\right\rangle$ as obtained for $J_{H} / U=0.3$ in the MF approximation (dashed lines) and with the quantum corrections calculated in RPA (full lines). The splitting of lines for $E_{z} / J>0$ corresponds to the MOAFF phase with two different hole densities $\left\langle n_{x}\right\rangle_{A} \neq\left\langle n_{x}\right\rangle_{B}$ on the ions belonging to two sublattices (see Fig. 3).

case of $J_{H}=0$ one finds, however, that the contribution from the lower mode either stays approximately constant (in the AFxx phase), or even decreases (in the AFzz phase) when the line of the collapsing LRO is approached at $\left|E_{z}\right| \rightarrow 0$ (Table I). This latter behavior shows again that the coupling between the spin-wave and spin-and-orbital-wave excitations is of crucial importance. ${ }^{65}$ This is further illustrated by Fig. 17, which shows the renormalization of $\left\langle S_{z}\right\rangle$ as obtained when spin waves and spin-and-orbital waves are decoupled in the manner discussed above. One observes that significant reduction of $\left\langle S_{z}\right\rangle$ then sets in only very close to the actual divergence.

Also the orbital polarization is renormalized by the quantum fluctuations, but this is a rather mild effect not showing any instability, since this renormalization involves only the spin-and-orbital and the orbital excitation but not the spin excitation, which is the one participating most strongly in the lowest transverse mode that goes soft. This is seen in Fig. 18, where we show $\left\langle n_{x}\right\rangle$, the occupation of the $|x\rangle$ orbital, again for $J_{H} / U=0.3$, both at the MF level as well as including the

TABLE II. Individual contributions to the quantum corrections of the magnetic order parameter $\left\langle\delta \mathcal{S}^{z}\right\rangle$ in MO phases due to spin wave, $\left\langle\mathcal{S}^{-} \mathcal{S}^{+}\right\rangle$, and due to spin-and-orbital-wave excitations, $\left\langle\mathcal{K}^{-} \mathcal{K}^{+}\right\rangle$, and due to individual modes as labeled in Figs. 11 and $12,\left\langle\delta \mathcal{S}^{z}\right\rangle_{n}$, respectively. The values of the renormalized order parameter in RPA are given by $\left\langle\mathcal{S}^{z}\right\rangle_{\mathrm{RPA}}$.

\begin{tabular}{crcccccccc}
\hline \hline$J_{H} / U$ & $E_{z} / J$ & $\left\langle\mathcal{S}^{-} \mathcal{S}^{+}\right\rangle$ & $\left\langle\mathcal{K}^{-} \mathcal{K}^{+}\right\rangle$ & $\left\langle\delta \mathcal{S}^{z}\right\rangle_{1}$ & $\left\langle\delta \mathcal{S}^{z}\right\rangle_{2}$ & $\left\langle\delta \mathcal{S}^{z}\right\rangle_{3}$ & $\left\langle\delta \mathcal{S}^{z}\right\rangle_{4}$ & $\left\langle\delta \mathcal{S}^{z}\right\rangle$ & $\left\langle\mathcal{S}^{z}\right\rangle_{\mathrm{RPA}}$ \\
\hline 0.2 & 0.0 & 0.1350 & 0.0508 & 0.0114 & 0.0344 & 0.0709 & 0.0691 & 0.1858 & 0.3142 \\
0.3 & -2.0 & 0.2138 & 0.0323 & 0.0673 & 0.0646 & 0.0585 & 0.0557 & 0.2461 & 0.2539 \\
0.3 & -1.0 & 0.1338 & 0.0336 & 0.0411 & 0.0025 & 0.0547 & 0.0691 & 0.1674 & 0.3326 \\
0.3 & 0.0 & 0.0918 & 0.0354 & 0.0122 & 0.0241 & 0.0425 & 0.0485 & 0.1273 & 0.3727 \\
0.3 & 1.0 & 0.1095 & 0.0323 & 0.0285 & 0.0041 & 0.0684 & 0.0408 & 0.1418 & 0.3582 \\
0.3 & 2.0 & 0.1330 & 0.0328 & 0.0327 & 0.0076 & 0.0754 & 0.0502 & 0.1658 & 0.3342 \\
0.3 & 3.0 & 0.1664 & 0.0329 & 0.0465 & 0.0146 & 0.0738 & 0.0644 & 0.1993 & 0.3007 \\
0.4 & -3.0 & 0.2144 & 0.0232 & 0.0876 & 0.0958 & 0.0294 & 0.0249 & 0.2376 & 0.2624 \\
0.4 & -2.0 & 0.1373 & 0.0258 & 0.0552 & 0.0145 & 0.0453 & 0.0482 & 0.1631 & 0.3369 \\
0.4 & -1.0 & 0.0928 & 0.0269 & 0.0370 & 0.0020 & 0.0302 & 0.0505 & 0.1197 & 0.3803 \\
0.4 & 0.0 & 0.0647 & 0.0274 & 0.0224 & 0.0080 & 0.0257 & 0.0360 & 0.0921 & 0.4079 \\
0.4 & 1.0 & 0.0776 & 0.0254 & 0.0258 & 0.0038 & 0.0494 & 0.0240 & 0.1030 & 0.3970 \\
0.4 & 2.0 & 0.0924 & 0.0258 & 0.0292 & 0.0063 & 0.0552 & 0.0276 & 0.1182 & 0.3818 \\
0.4 & 3.0 & 0.1117 & 0.0259 & 0.0363 & 0.0104 & 0.0590 & 0.0319 & 0.1376 & 0.3624 \\
\hline \hline
\end{tabular}


TABLE III. The mean-field energy $E_{\mathrm{MF}}$, the quantum energy correction due to transverse modes and due to longitudinal modes, $\delta E_{t}$ and $\delta E_{l}$, respectively, and the ground-state energy in RPA, $E_{\mathrm{RPA}}$ (all in the units of $J$ ). The labels FFA and AFF indicate the way of staggering of FM planes in the MO phases with A-AF order.

\begin{tabular}{|c|c|c|c|c|c|c|}
\hline$J_{H} / U$ & $E_{z} / J$ & $E_{\mathrm{MF}}$ & $\delta E_{t}$ & $\delta E_{l}$ & $E_{\mathrm{RPA}}$ & phase \\
\hline 0.0 & -2.0 & -4.0000 & 0.6440 & 0.0 & -4.6440 & AFzz \\
\hline 0.0 & -1.0 & -3.5000 & 0.6700 & 0.0 & -4.1700 & AFzz \\
\hline 0.0 & 1.0 & -3.5000 & 0.7073 & 0.0 & -4.2073 & AFxx \\
\hline 0.0 & 2.0 & -4.0000 & 0.6399 & 0.0 & -4.6399 & AFxx \\
\hline 0.1 & -2.0 & -3.9250 & 0.6354 & 0.0008 & -4.5612 & AFzz \\
\hline 0.1 & -1.0 & -3.4250 & 0.6735 & 0.0021 & -4.1006 & AFzz \\
\hline 0.1 & 1.0 & -3.4250 & 0.7344 & 0.0020 & -4.1614 & AFxx \\
\hline 0.1 & 2.0 & -3.9250 & 0.6384 & 0.0008 & -4.5642 & AFxx \\
\hline 0.2 & -3.0 & -4.3500 & 0.6082 & 0.0024 & -4.9606 & AFzz \\
\hline 0.2 & -2.0 & -3.8500 & 0.6328 & 0.0042 & -4.4870 & AFzz \\
\hline 0.2 & -1.0 & -3.4769 & 0.3964 & 0.0009 & -3.8742 & FFA \\
\hline 0.2 & 0.0 & -3.2558 & 0.2992 & 0.0028 & -3.5577 & FFA \\
\hline 0.2 & 1.0 & -3.3543 & 0.3437 & 0.0010 & -3.6990 & $\mathrm{AFF}$ \\
\hline 0.2 & 2.0 & -3.4769 & 0.3962 & 0.0005 & -3.8738 & $\mathrm{AFF}$ \\
\hline 0.2 & 2.0 & -3.8500 & 0.6472 & 0.0041 & -4.5013 & AFxx \\
\hline 0.3 & -3.0 & -4.2750 & 0.6052 & 0.0062 & -4.8864 & AFzz \\
\hline 0.3 & -3.0 & -4.2272 & 0.5252 & 0.0194 & -4.7717 & FFA \\
\hline 0.3 & -2.0 & -3.7750 & 0.6419 & 0.0134 & -4.4303 & AFzz \\
\hline 0.3 & -2.0 & -3.8651 & 0.3944 & 0.0037 & -4.2632 & FFA \\
\hline 0.3 & -1.0 & -3.5892 & 0.3040 & 0.0019 & -3.8951 & FFA \\
\hline 0.3 & 0.0 & -3.3996 & 0.2335 & 0.0054 & -3.6384 & FFA \\
\hline 0.3 & 1.0 & -3.4836 & 0.2664 & 0.0031 & -3.7531 & $\mathrm{AFF}$ \\
\hline 0.3 & 2.0 & -3.5892 & 0.3038 & 0.0016 & -3.8947 & $\mathrm{AFF}$ \\
\hline 0.3 & 2.0 & -3.7750 & 0.6768 & 0.0134 & -4.4652 & AFxx \\
\hline 0.3 & 3.0 & -3.7164 & 0.3459 & 0.0015 & -4.0638 & $\mathrm{AFF}$ \\
\hline 0.3 & 3.0 & -4.2750 & 0.5773 & 0.0063 & -4.8586 & AFxx \\
\hline 0.3 & 10.0 & -7.7750 & 0.4048 & 0.0014 & -8.1812 & AFxx \\
\hline
\end{tabular}

RPA quantum fluctuations, calculated from an expression similar to Eq. (5.4), e.g., in the AFxx phase from

$$
\left\langle n_{i x}\right\rangle=1-4\left\langle T_{i z x} T_{i x z}\right\rangle-\left\langle K_{i}^{-} K_{i}^{+}\right\rangle .
$$

Especially in the MOFFA and MOAFF phases the deviation from the classical value of $\theta$ as given by Eq. (3.11) and by Eqs. (3.14) and (3.14), respectively, is small. Only in the AFxx phase a significant admixture of $|z\rangle$ occupancy could occur close to the regime where this phase becomes unstable due to the divergence of $\left\langle S^{z}\right\rangle_{\mathrm{RPA}}$.

The reduction of $\left\langle S^{z}\right\rangle_{\mathrm{RPA}}$ in the MOFFA/MOAFF phases (Table II), described by a relation similar to Eq. (5.4), is in general weaker than that in the G-AF phases. This is understandable, as the quantum fluctuations contribute here only from a single AF direction, while the FM order in the planes does not allow for excitations which involve spin flips and stabilizes the LRO of A-AF type. For fixed $J_{H}$ one finds increasing quantum corrections $\delta\left\langle S^{z}\right\rangle$ when the lines of phase transitions towards the AF phases are approached. These corrections increase faster with increasing $\left|E_{z}\right|$ in the MOFFA phase, as the increasing occupancy of the $|z\rangle$ orbital makes the AF interaction stronger there than in the MOAFF phase, where the occupancy of the $|x\rangle$ orbital increases slower roughly by a factor of two. This qualitative difference between these two A-AF phases may be seen in Fig. 18. As in the G-AF phases, we find that the two lower-energy modes give the larger contribution to the renormalization of the order parameter. The spin-and-orbital fluctuation $\left\langle\mathcal{K}_{i}^{-} \mathcal{K}_{i}^{+}\right\rangle$remains almost independent of $E_{z}$, but increases with decreasing values of $J_{H}$. Thus we conclude that the collapse of the LRO in the A-AF (MO) phases is primarily due to increasing spin fluctuations $\left\langle\mathcal{S}_{i}^{-} \mathcal{S}_{i}^{+}\right\rangle$, while the spinand-orbital fluctuations become of equal importance only when the multicritical point of the Kugel-Khomskii model $M=\left(E_{z}, J_{H}\right)=(0,0)$ is approached.

The representative quantum corrections to the groundstate energy are given in Table III. First of all, these corrections are larger by roughly a factor of 2 in the G-AF phases (AFxx and AFzz) than in the A-AF phases (MOFFA and MOAFF/MOFAF). We believe that this is a generic difference between the quantum corrections in the A-type and G-type AF phases, with the latter stabilized more due to the spin fluctuations contributing at all the bonds. Therefore the G-AF phases win over the A-AF ones near the transition lines, as, for example, found at $J_{H} / U=2.0$ and $E_{z} / J=0.2$. However, one should keep in mind that the energy alone 


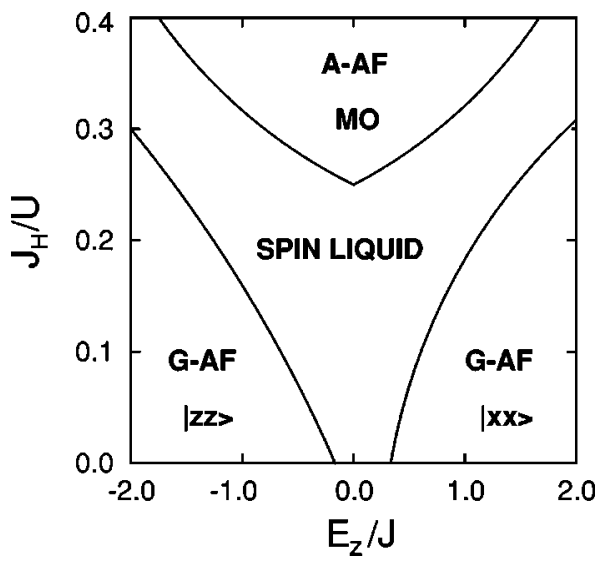

FIG. 19. Schematic phase diagram of the spin-orbital model including quantum fluctuations. The spin liquid phase is expected to separate the AF phases with different types of magnetic LRO: G-AF phases with either $d_{x^{2}-y^{2}}(|x x\rangle)$ or $d_{3 z^{2}-r^{2}}(|z z\rangle)$ orbital occupied on both sublattices from the A-AF phases with mixed orbitals (MO's) ordered on two sublattices.

does not suffice for the stability of a particular phase in RPA, since the MF value of the order parameter, $\left\langle S^{z}\right\rangle$, has to remain larger than the respective quantum correction, $\delta\left\langle S^{z}\right\rangle$. Second, the 2D AFxx phase is characterized by larger quantum corrections than the strongly anisotropic AFzz phase at the same values of $J_{H} / U$ and $\left|E_{z}\right| / J$. The same observation was made before at the multicritical point $M=\left(E_{z}, J_{H}\right)$ $=(0,0) \cdot{ }^{65}$ This is not surprising since the $2 \mathrm{D}$ HAF is already quite close to the disordered spin state. We note that the energy gain due to quantum fluctuations of $0.423 \mathrm{~J}$ (obtained for the actual interactions of $\frac{9}{4} J$ in a $2 \mathrm{D} \mathrm{HAF}$ ) is there considerably smaller than the values of $\delta E$ of the order of $0.65 \mathrm{~J}$ reported in Table III.

Finally, we note that the dominating contribution to the quantum corrections to the energy comes from the transverse excitations. The longitudinal excitations do not contribute at all at $J_{H} / U=0$, where these modes are dispersionless. Otherwise, the orbital excitations have always a significantly smaller dispersion than the value of the orbital gap in the spectrum, and the resulting quantum corrections are therefore almost negligible.

\section{SUMMARY AND CONCLUSIONS}

Summarizing, we have presented here the case that a generic (Kugel-Khomskii) model for the dynamics of an orbitally degenerate $\mathrm{MHI}$ is characterized by a number of peculiar features. In this paper we have followed a semiclassical strategy. Assuming that the ground state exhibits some particular classical spin and orbital order, the stability of this order can be investigated by considering the Gaussian fluctuations around this state. In this way we find that in various regimes of the zero-temperature phase diagram, conventional order is defeated by the quantum fluctuations, and we expect a qualitative phase diagram as shown in Fig. 19.

In the first place, near the transition lines between the different phases modes soften, and these soft modes cause the zero-point fluctuations to diverge. This is not dissimilar from the general theme associated with the geometrically frustrated quantum spin models, like the $J_{1}-J_{2}-J_{3}$ model. ${ }^{41}$
A significant difference is that in the present case the source of the problems is distinct: it is associated with the difficulty to satisfy simultaneously the requirements for a stable spin and orbital order. The cause of the frustration is dynamical instead of geometrical.

The most interesting feature is the point at the origin of the phase diagram. On the classical level it is a point in the zero-temperature phase diagram where a quasi-1D antiferromagnet (MOFFA phase), a 2D antiferromagnet (AFxx phase), and a mildly anisotropic 3D antiferromagnet (AFzz phase) become degenerate (Fig. 4). In fact, these possibilities make up only an infinitesimal fraction of the total degeneracy characterizing this special point. In addition, the orbitals can be freely rotated on every site, if the spins form a 3D antiferromagnet. Likewise, the phase diagram of Fig. 19 is highly incomplete. Next to $E_{z}$, there exist an infinity of other axes emerging from this special point, all corresponding with distinct ways of explicit local symmetry breaking in the orbital sector. One can either call this point an infinite-critical point, or a point of perfect dynamical frustration, or, finally, a point where local symmetry is dynamically generated.

The obvious problem is that the above wisdom applies only when quantum mechanics does not play a role. Physical reality is different, and since the classical limit is pathological, quantum mechanics is bound to take over. Although we have not found a way to make the case precise, it appears to us that the local symmetry referred to in the previous paragraph exists only in the classical limit. For this to be active on the quantum level, it should be that the true ground state is also highly degenerate. Although we did not prove the uniqueness of the quantum ground state, so much is clear that the classical local symmetry gets lifted at the moment that quantum fluctuations become significant: the cancellations occur only if the spins are fully classical. Regardless the nature of the true ground state, it is generated by a quantum order-out-of-disorder mechanism. ${ }^{42}$

The first possibility is a straightforward order-out-ofdisorder physics: the quantum fluctuations affect the energies of the various classical states in different ways, thereby breaking the classical degeneracy. One of the saddle points might get uniquely favored and this is what is suggested in Ref. 72, where it was argued that the AFzz phase becomes the ground state at the origin of the phase diagram. Although this is a credible possibility, one would have to demonstrate that the other possibilities are less favored, and moreover, we have shown elsewhere ${ }^{65}$ that the actual calculation by Khaliullin and Oudovenko ${ }^{72}$ is flawed. The case is still open.

Yet another possibility is unconventional spin and orbital order which is in a sense dual to the orbital and spin (anti)ferromagnetism characterizing the "classical" order: spin-orbital (resonating) valence bond (R)VB states. We demonstrated before ${ }^{6}$ that these straightforward generalizations of the spin RVB states, well known from the study of quantum spin-problems, appear as exceptionally stable. In a next publication we will further elaborate on these matters. ${ }^{76}$

The status of both proposals is rather unsure: they rely at best on the variational principle and the true vacuum can still be completely different. In this regard, some recent experiments on the system $\mathrm{LiNiO}_{2}$ are quite interesting. ${ }^{80}$ In this material a Mott insulator seems to be realized, characterized by a low spin $(S=1 / 2) e_{g}$ degenerate $\mathrm{Ni}(\mathrm{III})$ state. One 
would naively expect this system to be unstable towards a collective Jahn-Teller distortion, accompanied by spin ordering. This indeed happens in the closely related system $\mathrm{NaNiO}_{2}$, but in $\mathrm{LiNiO}_{2}$ ordering phenomena are completely absent, ${ }^{81}$ a peculiarity pointed out long ago. ${ }^{82}$ Instead, some quantum-critical state appears to be present, characterized by power-law behavior of physical quantities, carrying unusual exponents. Pending the magnitude of the Li-mediated kinetic exchange $\left(J_{\mathrm{Li}}\right)$, one can view this system as either disconnected triangular layers of $\mathrm{Ni}(\mathrm{III})$ ions (vanishing $J_{\mathrm{Li}}$ ), or as interpenetrating cubic lattices of these ions which are described by the Kugel-Khomskii Hamiltonian (large $\left.J_{\mathrm{Li}}\right){ }^{6}$ Hence the peculiar state seen in the experiments can either originate in some phenomenon associated with the triangular layers, ${ }^{83}$ but it could also be related to the matters discussed in this paper.

It is easy to settle this issue experimentally. Compare $\mathrm{NaNiO}_{2}$ and $\mathrm{LiNiO}_{2}$; if the physics of the quantum disorder in the latter has to do with the (111) layers, one would expect on general grounds that in order to stabilize an ordered state, the effective dimensionality has to be increased, of course assuming that the basics of the electronic structure (such like covalency) do not change appreciably. Hence in this layer scenario one would expect stronger layer-layer interactions in $\mathrm{NaNiO}_{2}$ as compared to $\mathrm{LiNiO}_{2}$, following the standard result of quantum field theory that fluctuations increase upon lowering dimensionality. This standard wisdom does not apply to the Kugel-Khomskii model, however. The fluctuations find their origin in a dynamical frustration, and this frustration is only present in three space dimensions. Hence if the disorder in $\mathrm{LiNiO}_{2}$ is caused by the physics discussed in this paper, its quantum magnetism should be rather isotropic in 3D space, while $\mathrm{NaNiO}_{2}$ should be more 2D. It is noticed that according to elementary quantum chemistry $\mathrm{Li}$ ions should be more effective in mediating kinetic exchange than $\mathrm{Na}$ ions.

\section{ACKNOWLEDGMENTS}

We thank P. Horsch, D. I. Khomskii, J. Richter, and M. Takano for valuable discussions. A.M.O. acknowledges support by the Committee of Scientific Research (KBN) of Poland, Project No. 2 P03B 17514.

\section{APPENDIX A: DERIVATION OF THE SPIN-ORBITAL MODEL}

The derivation of the effective interactions between two $d^{9}$ ions at sites $i$ and $j$ takes the simplest form for a bond $\langle i j\rangle$ oriented along the $c$ axis. In that case the only nonvanishing hopping element is that between the two $|z\rangle$ orbitals on the neighboring sites, and thus the orbital occupancies in the initial and final $d_{i}^{9} d_{j}^{9}$ states have to be identical (apart from a possible simultaneous and opposite spin flip at both sites). The possible initial states are described by a direct product of the total spin state, either a triplet $(S=1)$ or a singlet $(S$ $=0)$, and the orbital configuration, which takes one of four possibilities: $\left|x_{i} x_{j}\right\rangle,\left|x_{i} z_{j}\right\rangle,\left|z_{i} x_{j}\right\rangle$, or $\left|z_{i} z_{j}\right\rangle$. Moreover, the effective interaction vanishes if the holes occupy the $\left|x_{i} x_{j}\right\rangle$ configuration. The total spin per two sites is conserved in the $d_{i}^{9} d_{j}^{9} \rightarrow d_{i}^{10} d_{j}^{8}$ excitation process, and therefore the spin de- pendence of the resulting second-order Hamiltonian can be expressed in terms of the projection operators on the total spin states: $\left(\frac{3}{4}+\vec{S}_{i} \cdot \vec{S}_{j}\right)$ for the triplet, and $\left(\frac{1}{4}-\vec{S}_{i} \cdot \vec{S}_{j}\right)$ for the singlet.

The general form of the effective Hamiltonian may be derived from the formula which includes all possible virtual transitions to the excited $d^{8} d^{10}$ configurations,

$$
H_{\langle i j\rangle}=-\sum_{n, \alpha \beta} \frac{t^{2}}{\varepsilon_{n}} Q_{S(i, j)} P_{i \alpha} P_{j \beta},
$$

where $t$ stands for the $z-z$ hopping along the $c$ axis, $Q_{S(i, j)}$ is the projection operator on the total spin state, and $P_{i \alpha}$ is the projection operator on the orbital state $\alpha$ at site $i$, while $\varepsilon_{n}$ stands for the excitation energies given by Eqs. (2.5). The orbital projection operators on $|x\rangle$ and $|z\rangle$ orbital in the initial and final state of the $d^{9}$ configuration at site $i$ are, respectively,

$$
\begin{aligned}
& P_{i x}=|i x\rangle\langle i x|=\frac{1}{2}+\tau_{i}^{c}, \\
& P_{i z}=|i z\rangle\langle i z|=\frac{1}{2}-\tau_{i}^{c},
\end{aligned}
$$

where $\tau_{i}^{c}$ is defined as in Eqs. (2.12).

Therefore one finds from Eq. (A1) for a bond $\langle i j\rangle$ along the $c$ direction

$$
\begin{aligned}
H_{\langle i j\rangle}= & -\frac{t^{2}}{\varepsilon\left({ }^{3} A_{2}\right)}\left(\vec{S}_{i} \cdot \vec{S}_{j}+\frac{3}{4}\right)\left(P_{i x} P_{j z}+P_{i z} P_{j x}\right) \\
& +\frac{t^{2}}{\varepsilon\left({ }^{1} E_{\epsilon}\right)}\left(\vec{S}_{i} \cdot \vec{S}_{j}-\frac{1}{4}\right)\left(P_{i x} P_{j z}+P_{i z} P_{j x}\right) \\
& +\left[\frac{t^{2}}{\varepsilon\left({ }^{1} E_{\theta}\right)}+\frac{t^{2}}{\varepsilon\left({ }^{1} A_{1}\right)}\right]\left(\vec{S}_{i} \cdot \vec{S}_{j}-\frac{1}{4}\right) 2 P_{i z} P_{j z} .
\end{aligned}
$$

While the magnetic interactions due to the first two terms in Eq. (A3) cancel each other in the limit of $\eta \rightarrow 0$, the last term favors AF spin orientation. We recognize that Hamiltonian (A3) describes the superexchange with the superexchange constant of $4 t^{2} / U .{ }^{57}$ However, for convenience we define the energy unit as $J=t^{2} / U$ in the present paper. Although the form (A3) might in principle be used for further analysis, we prefer to make an expansion of the excitation energies $\varepsilon_{n}$ in the denominators for small $J_{H}$, and use $\eta=J_{H} / U$ (2.7) as a parameter which quantifies the Hund's rule exchange. ${ }^{50}$ Using the explicit form of the orbital projection operators $P_{i \alpha}$ (A2) this results in the following form of the effective Hamiltonian for the bond $\langle i j\rangle \|_{c}$ :

$$
\begin{aligned}
H_{\langle i j\rangle}= & J\left[(1+\eta)\left(\vec{S}_{i} \cdot \vec{S}_{j}+\frac{3}{4}\right)-\left(\vec{S}_{i} \cdot \vec{S}_{j}-\frac{1}{4}\right)\right] \\
& \times\left[\left(\tau_{i}^{c}+\frac{1}{2}\right)\left(\tau_{j}^{c}-\frac{1}{2}\right)+\left(\tau_{i}^{c}-\frac{1}{2}\right)\left(\tau_{j}^{c}+\frac{1}{2}\right)\right] \\
& +4 J\left(1-\frac{1}{2} \eta\right)\left(\vec{S}_{i} \cdot \vec{S}_{j}-\frac{1}{4}\right)\left(\tau_{i}^{c}-\frac{1}{2}\right)\left(\tau_{j}^{c}-\frac{1}{2}\right),
\end{aligned}
$$

which may be further simplified to the form 


$$
\begin{aligned}
H_{\langle i j\rangle}= & J\left\{\left(4 \vec{S}_{i} \cdot \vec{S}_{j}+1\right)\left(\tau_{i}^{c}-\frac{1}{2}\right)\left(\tau_{j}^{c}-\frac{1}{2}\right)+\tau_{i}^{c}+\tau_{j}^{c}-1\right. \\
& +\eta\left(\vec{S}_{i} \cdot \vec{S}_{j}\right)\left(\tau_{i}^{c}+\tau_{j}^{c}-1\right)+\frac{1}{2} \eta\left[\left(\tau_{i}^{c}-\frac{1}{2}\right)\left(\tau_{j}^{c}-\frac{1}{2}\right)\right. \\
& \left.\left.+3\left(\tau_{i}^{c} \tau_{j}^{c}-\frac{1}{4}\right)\right]\right\} .
\end{aligned}
$$

The first line represents the AF superexchange interactions $\propto J$, while the other two lines describe the weaker FM interactons $\propto J \eta$, and stand for the corrections due to the multiplet splittings of the $d^{8}$ excited states.

It is straightforward to verify that the above form of the effective Hamiltonian simplifies in the limit of occupied $|z\rangle$ orbitals to

$$
H_{\langle i j\rangle}=4 J\left(1-\frac{1}{2} \eta\right)\left(\vec{S}_{i} \cdot \vec{S}_{j}-\frac{1}{4}\right),
$$

and one recognizes the same constant $-\frac{1}{4}$, and the same superexchange interaction $4 J=4 t^{2} / U$ as in the $t$ - $J$ model at half filling. ${ }^{57}$ However, the effective superexchange is somewhat reduced by the factor $\left(1-\frac{1}{2} \eta\right)$ in the presence of the Hund's rule interaction.

The effective interactions on the bonds within the $(a, b)$ planes may be now obtained by rotating the orbital operators $\tau_{i}^{c}$ in Eq. (A4) by $\pi / 2$ to the cubic axes $a$ and $b$ which generates the orbital operators $\tau_{i}^{a}$ and $\tau_{i}^{b}$ (2.12), respectively. This results in a nontrivial coupling between the orbital and spin degrees of freedom, as given in Eq. (2.10). We note that in the case of a single $s$ orbital per site, it would suffice to rotate instead the simpler projected form (A6), which would give the same superexchange interaction in any direction.

\section{APPENDIX B: COMMUTATION RULES IN THE SO(4) ALGEBRA FOR THE SPIN-ORBITAL MODEL}

In order to illustrate the full algebraic structure of our problem, we present here the so(4) commutators between the various excitation operators which are needed for calculating the excitation spectra in Sec. IV. As the operators defined on different sites commute, we only specify the on-site commutators.

The spin operators fulfill the usual relations for each orbital $\alpha=x, z$,

$$
\begin{aligned}
& {\left[S_{i \alpha \alpha}^{+}, S_{i \alpha \alpha}^{z}\right]=-S_{i \alpha \alpha}^{+},} \\
& {\left[S_{i \alpha \alpha}^{+}, S_{i \alpha \alpha}^{-}\right]=2 S_{i \alpha \alpha}^{z} .}
\end{aligned}
$$

Their commutators with the other operators which describe either spin-and-orbital (transverse), or orbital (longitudinal, i.e., excitonic) excitations are responsible for the coupling between spin- and spin-and-orbital excitations (here $\alpha \neq \beta$ ),

$$
\begin{gathered}
{\left[S_{i \alpha \alpha}^{+}, K_{i \alpha \beta}^{z}\right]=-\frac{1}{2} K_{i \alpha \beta}^{+},} \\
{\left[S_{i \alpha \alpha}^{+}, K_{i \beta \alpha}^{z}\right]=-\frac{1}{2} K_{i \beta \alpha}^{+},} \\
{\left[S_{i \alpha \alpha}^{+}, K_{i \alpha \beta}^{-}\right]=\left(K_{i \alpha \beta}^{z}+T_{i \alpha \beta}\right),} \\
{\left[S_{i \alpha \alpha}^{+}, K_{i \beta \alpha}^{-}\right]=\left(K_{i \beta \alpha}^{z}-T_{i \beta \alpha}\right),} \\
{\left[S_{i \alpha \alpha}^{+}, T_{i \alpha \beta}\right]=\frac{1}{2} K_{i \alpha \beta}^{+},} \\
{\left[S_{i \alpha \alpha}^{+}, T_{i \beta \alpha}\right]=-\frac{1}{2} K_{i \beta \alpha}^{+},}
\end{gathered}
$$

while they commute with the orbital-polarization operator,

$$
\left[S_{i \alpha \alpha}^{+}, n_{i-}\right]=0 \text {. }
$$

The operators for spin-and-orbital excitations have the following commutators: (i) with the spin operators,

$$
\begin{gathered}
{\left[K_{i \alpha \beta}^{+}, S_{i \alpha \alpha}^{z}\right]=-\frac{1}{2} K_{i \alpha \beta}^{+},} \\
{\left[K_{i \alpha \beta}^{+}, S_{i \beta \beta}^{z}\right]=-\frac{1}{2} K_{i \alpha \beta}^{+},} \\
{\left[K_{i \alpha \beta}^{+}, S_{i \alpha \alpha}^{-}\right]=K_{i \alpha \beta}^{z}-T_{i \alpha \beta},} \\
{\left[K_{i \alpha \beta}^{+}, S_{i \beta \beta}^{-}\right]=K_{i \beta \alpha}^{z}+T_{i \beta \alpha},}
\end{gathered}
$$

(ii) with the spin-and-orbital operators,

$$
\begin{gathered}
{\left[K_{i \alpha \beta}^{+}, K_{i \alpha \beta}^{z}\right]=0,} \\
{\left[K_{i \alpha \beta}^{+}, K_{i \beta \alpha}^{z}\right]=-\frac{1}{2}\left(S_{i \alpha \alpha}^{+}+S_{i \beta \beta}^{+}\right),} \\
{\left[K_{i \alpha \beta}^{+}, K_{i \alpha \beta}^{-}\right]=0,} \\
{\left[K_{i \alpha \beta}^{+}, K_{i \beta \alpha}^{-}\right]=\frac{1}{2}\left(n_{i \alpha}-n_{i \beta}\right)+S_{i \alpha \alpha}^{z}+S_{i \beta \beta}^{z},} \\
{\left[K_{i \alpha \beta}^{+}, T_{i \alpha \beta}\right]=0,} \\
{\left[K_{i \alpha \beta}^{+}, T_{i \beta \alpha}\right]=-\frac{1}{2}\left(S_{i \alpha \alpha}^{+}-S_{i \beta \beta}^{+}\right),}
\end{gathered}
$$

and (iii) with the orbital-polarization operator,

$$
\begin{aligned}
& {\left[K_{i x z}^{+}, n_{i-}\right]=-K_{i x z}^{+},} \\
& {\left[K_{i z x}^{+}, n_{i-}\right]=+K_{i z x}^{+} .}
\end{aligned}
$$

The relevant excitonic operators in the symmetry-broken state (4.11) commute with the above spin-transverse operators, $S_{i \alpha \alpha}^{+}$and $K_{i \alpha \beta}^{+}$, and give the following commutators with the remaining spin-longitudinal operators,

$$
\begin{gathered}
{\left[T_{i \alpha \beta \sigma}, S_{i \alpha \alpha}^{z}\right]=-\frac{1}{2} \lambda_{\sigma} T_{i \alpha \beta \sigma},} \\
{\left[T_{i \alpha \beta \sigma}, S_{i \beta \beta}^{z}\right]=+\frac{1}{2} \lambda_{\sigma} T_{i \alpha \beta \sigma},} \\
{\left[T_{i \alpha \beta \sigma}, K_{i \alpha \beta}^{z}\right]=0,} \\
{\left[T_{i \alpha \beta \sigma}, K_{i \beta \alpha}^{z}\right]=\frac{1}{2}\left(S_{i \alpha \alpha}^{+}+S_{i \beta \beta}^{+}\right)+\frac{1}{4} \lambda_{\sigma}\left(n_{i \alpha}-n_{i \beta}\right),} \\
{\left[T_{i \alpha \beta \sigma}, T_{i \alpha \beta}\right]=0,} \\
{\left[T_{i \alpha \beta \sigma}, T_{i \beta \alpha}\right]=\frac{1}{2} \lambda_{\sigma}\left(S_{i \alpha \alpha}^{+}+S_{i \beta \beta}^{+}\right)+\frac{1}{4}\left(n_{i \alpha}-n_{i \beta}\right),} \\
{\left[T_{i x z \sigma}, n_{i-}\right]=-T_{i x z \sigma},} \\
{\left[T_{i z x \sigma}, n_{i-}\right]=+T_{i z x \sigma},}
\end{gathered}
$$

where $\lambda_{\sigma}= \pm 1$ for $\sigma=\uparrow, \downarrow$. Therefore the subset of longitudinal operators $\left\{T_{i \alpha \beta \sigma}\right\}$ generates the excitations which do not couple to the transverse excitations.

\section{APPENDIX C: GREEN-FUNCTION EQUATIONS FOR SPIN AND ORBITAL EXCITATIONS}

Here we present the dynamical matrices obtained for the phases with LRO for the spin-orbital model (2.10). It is easy to verify that the presented dynamical matrices have an RPA structure and thus describe symmetric spectra with respect to $\omega=0$.

Let us start with the G-AF phases with either $|x\rangle$ or $|z\rangle$ 
orbitals occupied. The spin and spin-and-orbital excitations are determined from Eqs. (4.12) and (4.13) for the AFxx phase, and from Eqs. (4.34) and (4.35) for the AFzz phase. After using the translational symmetry and performing the familiar RPA decoupling procedure, ${ }^{70,71}$

$$
\left\langle\left\langle\mathcal{A}_{i} \mathcal{B}_{j} \mid \cdots\right\rangle\right\rangle \simeq\left\langle\mathcal{A}_{i}\right\rangle\left\langle\left\langle\mathcal{B}_{j} \mid \cdots\right\rangle\right\rangle+\left\langle\mathcal{B}_{j}\right\rangle\left\langle\left\langle\mathcal{A}_{i} \mid \cdots\right\rangle\right\rangle,
$$

where $i$ and $j$ refer to different sites, one finds a system of linear equations for the excitation energies. A straightforward but somewhat lengthy calculation shows that the same matrix with different coefficients describes the elementary excitations for both AF phases,

$$
\begin{aligned}
& \left(\begin{array}{cccc}
\lambda_{\alpha}-\bar{\omega}_{\vec{k}} & 0 & Q_{\alpha \vec{k}} & P_{\alpha \vec{k}} \\
0 & \tau_{\alpha}-\bar{\omega}_{\vec{k}} & P_{\alpha \vec{k}} & R_{\vec{k}} \\
-Q_{\alpha \vec{k}} & -P_{\alpha \vec{k}} & -\lambda_{\alpha}-\bar{\omega}_{\vec{k}} & 0 \\
-P_{\alpha \vec{k}} & -R_{\vec{k}} & 0 & -\tau_{\alpha}-\bar{\omega}_{\vec{k}}
\end{array}\right) \\
& \quad \times\left(\begin{array}{c}
\left\langle\left\langle S_{\vec{k} x x}^{+} \mid \cdots\right\rangle\right\rangle_{A} \\
\left\langle\left\langle K_{\vec{k} x z}^{+} \mid \cdots\right\rangle\right\rangle_{A} \\
\left\langle\left\langle S_{\vec{k} x x}^{-} \mid \cdots\right\rangle\right\rangle_{B} \\
\left\langle\left\langle K_{\vec{k} x z}^{-} \mid \cdots\right\rangle\right\rangle_{B}
\end{array}\right)=0,
\end{aligned}
$$

where $\bar{\omega}_{\vec{k}}$ is the frequency in units of $J$, i.e., $\bar{\omega}_{\vec{k}}=\omega_{\vec{k}} / J$. The constants $\lambda_{\alpha}$ and $\tau_{\alpha}$ and the $\vec{k}$-dependent functions $P_{\alpha \vec{k}}$ and $Q_{\alpha \vec{k}}$ depend on the considered AF phase and are specified in Sec. IV, while $R_{\vec{k}}=\frac{3}{2} \gamma_{+}(\vec{k})$. The solution for the eigenenergies is given by Eq. (4.19). As discussed in Sec. IV A, the same $4 \times 4$ matrix equation written down in Eq. (C2) for $\left\langle\left\langle S_{\vec{k} x x}^{+} \mid \cdots\right\rangle\right\rangle_{A}$, etc., describing the modes generated by the spin-raising operators, is also valid for the Green functions $\left\langle\left\langle S_{\vec{k} x x}^{-} \mid \cdots\right\rangle\right\rangle_{A}$, etc., describing the modes generated by the spin-lowering operators $\left\{S_{i x x}^{-}, K_{i x z}^{-}, S_{j x x}^{-}, K_{j x z}^{-}\right\}$, with $i \in A$ and $j \in B$, and all transverse modes are doubly degenerate.

The orbital (longitudinal) excitations correspond to exciting an electron from one orbital to the other without changing the spin direction. If $A(B)$ is an up (down) sublattice in the Néel state, the basis operators which define the modes are $\uparrow$-spin ( $\downarrow$-spin) orbital excitations, as introduced in Sec. IV. One finds the following eigenvalue problem using the RPA:

$$
\begin{gathered}
\left(\begin{array}{cccc}
u_{\alpha}-\bar{\zeta}_{\vec{k}} & 0 & +\rho_{\alpha \vec{k}} & +\rho_{\alpha \vec{k}} \\
0 & -u_{\alpha}-\bar{\zeta}_{\vec{k}} & -\rho_{\alpha \vec{k}} & -\rho_{\alpha \vec{k}} \\
-\rho_{\alpha \vec{k}} & -\rho_{\alpha \vec{k}} & -u_{\alpha}-\bar{\zeta}_{\vec{k}} & 0 \\
+\rho_{\alpha \vec{k}} & +\rho_{\alpha \vec{k}} & 0 & u_{\alpha}-\bar{\zeta}_{\vec{k}}
\end{array}\right) \\
\quad\left(\begin{array}{c}
\left\langle\left\langle T_{\vec{k} x z \uparrow} \mid \cdots\right\rangle\right\rangle_{A} \\
\left\langle\left\langle T_{\vec{k} z x \uparrow} \mid \cdots\right\rangle\right\rangle_{A} \\
\left\langle\left\langle T_{\vec{k} x z \downarrow} \mid \cdots\right\rangle\right\rangle_{B} \\
\left\langle\left\langle T_{\vec{k} z x \downarrow} \mid \cdots\right\rangle\right\rangle_{B}
\end{array}\right)=0,
\end{gathered}
$$

where again $\bar{\zeta}_{\vec{k}}$ is in units of $J$, i.e., $\bar{\zeta}_{\vec{k}}=\zeta_{\vec{k}} / J$, and the quantities $u_{\alpha}$ and $\rho_{\alpha \vec{k}}$ depend on the considered G-AF phase.

The classical A-AF ground state is discussed here on the example of the MOFFA phase. It consists of four sublattices: two sublattices $(A$ and $B$ ) due to different orbital order in the $(a, b)$ planes (see Fig. 3 ), and two others $(C$ and $D)$ due to spins which alternate along the $c$ axis. The Hamiltonian (2.9) was first transformed to the new operators defined by Eqs. (4.45) and (4.46). For the bonds $\langle i j\rangle \|(a, b)$ with $i \in A(C)$ and $j \in B(D)$ one finds

$$
\begin{aligned}
\mathcal{H}_{\|}= & \frac{1}{4} J \sum_{\langle i j\rangle}\left[( 1 - \frac { 1 } { 2 } \eta ) \left(\left[(2-\cos 2 \theta) \overrightarrow{\mathcal{S}}_{i \mu \mu}+(2+\cos 2 \theta) \overrightarrow{\mathcal{S}}_{i \nu \nu}+\sin 2 \theta \overrightarrow{\mathcal{K}}_{i}\right]\left[(2-\cos 2 \theta) \overrightarrow{\mathcal{S}}_{j \mu \mu}+(2+\cos 2 \theta) \overrightarrow{\mathcal{S}}_{j \nu \nu}-\sin 2 \theta \overrightarrow{\mathcal{K}}_{i}\right]\right.\right. \\
& +3\left[\sin 2 \theta\left(\overrightarrow{\mathcal{S}}_{i \mu \mu}-\overrightarrow{\mathcal{S}}_{i \nu \nu}\right)+\cos 2 \theta \overrightarrow{\mathcal{K}}_{i}\right]\left[\sin 2 \theta\left(\overrightarrow{\mathcal{S}}_{j \mu \mu}-\overrightarrow{\mathcal{S}}_{j \nu \nu}\right)+\cos 2 \theta \overrightarrow{\mathcal{K}}_{j}\right]+\lambda_{i j} \sqrt{3}\left\{\left[(2-\cos 2 \theta) \overrightarrow{\mathcal{S}}_{i \mu \mu}+(2+\cos 2 \theta) \overrightarrow{\mathcal{S}}_{i \nu \nu}\right.\right. \\
& \left.+\sin 2 \theta \overrightarrow{\mathcal{K}}_{i}\right]\left[\sin 2 \theta\left(\overrightarrow{\mathcal{S}}_{j \mu \mu}-\overrightarrow{\mathcal{S}}_{j \nu \nu}\right)-\cos 2 \theta \overrightarrow{\mathcal{K}}_{j}\right]-\left[\sin 2 \theta\left(\overrightarrow{\mathcal{S}}_{i \mu \mu}-\overrightarrow{\mathcal{S}}_{i \nu \nu}\right)+\cos 2 \theta \overrightarrow{\mathcal{K}}_{i}\right]\left[(2-\cos 2 \theta) \overrightarrow{\mathcal{S}}_{j \mu \mu}+(2+\cos 2 \theta) \overrightarrow{\mathcal{S}}_{j \nu \nu}\right. \\
& \left.\left.\left.-\sin 2 \theta \overrightarrow{\mathcal{K}}_{j}\right]\right\}\right)+\frac{1}{2} \eta\left(\left[\cos 2 \theta\left(\overrightarrow{\mathcal{S}}_{i \mu \mu}-\overrightarrow{\mathcal{S}}_{i \nu \nu}\right)-\sin 2 \theta \overrightarrow{\mathcal{K}}_{i}\right]\left[\cos 2 \theta\left(\overrightarrow{\mathcal{S}}_{j \mu \mu}-\overrightarrow{\mathcal{S}}_{j \nu \nu}\right)+\sin 2 \theta \overrightarrow{\mathcal{K}}_{j}\right]-3\left[\sin 2 \theta\left(\overrightarrow{\mathcal{S}}_{i \mu \mu}-\overrightarrow{\mathcal{S}}_{i \nu \nu}\right)\right.\right. \\
& \left.+\cos 2 \theta \overrightarrow{\mathcal{K}}_{i}\right]\left[\sin 2 \theta\left(\overrightarrow{\mathcal{S}}_{j \mu \mu}-\overrightarrow{\mathcal{S}}_{j \nu \nu}\right)-\cos 2 \theta \overrightarrow{\mathcal{K}}_{j}\right]-\lambda_{i j} \sqrt{3}\left\{[ \operatorname { c o s } 2 \theta ( \vec { \mathcal { S } } _ { i \mu \mu } - \vec { \mathcal { S } } _ { i \nu \nu } ) - \operatorname { s i n } 2 \theta \vec { \mathcal { K } } _ { i } ] \left[\sin 2 \theta\left(\overrightarrow{\mathcal{S}}_{j \mu \mu}-\overrightarrow{\mathcal{S}}_{j \nu \nu}\right)\right.\right. \\
& \left.\left.\left.-\cos 2 \theta \overrightarrow{\mathcal{K}}_{j}\right]+\left[\sin 2 \theta\left(\overrightarrow{\mathcal{S}}_{i \mu \mu}-\overrightarrow{\mathcal{S}}_{i \nu \nu}\right)+\cos 2 \theta \overrightarrow{\mathcal{K}}_{i}\right]\left[\cos 2 \theta\left(\overrightarrow{\mathcal{S}}_{j \mu \mu}-\overrightarrow{\mathcal{S}}_{j \nu \nu}\right)-\sin 2 \theta \overrightarrow{\mathcal{K}}_{j}\right]\right\}\right)-2 \eta \overrightarrow{\mathcal{S}}_{i} \overrightarrow{\mathcal{S}}_{j}+(1+2 \eta)\left\{\left(\cos 2 \theta \overrightarrow{\mathcal{N}}_{i}\right.\right. \\
& \left.-\sin 2 \theta \overrightarrow{\mathcal{T}}_{i}\right)\left(\cos 2 \theta \overrightarrow{\mathcal{N}}_{j}+\sin 2 \theta \overrightarrow{\mathcal{T}}_{j}\right)-3\left(\sin 2 \theta \overrightarrow{\mathcal{N}}_{i}+\cos 2 \theta \overrightarrow{\mathcal{T}}_{i}\right)\left(\sin 2 \theta \overrightarrow{\mathcal{N}}_{j}-\cos 2 \theta \overrightarrow{\mathcal{T}}_{j}\right)-\lambda_{i j} \sqrt{3}\left[\left(\cos 2 \theta \overrightarrow{\mathcal{N}}_{i}-\sin 2 \theta \overrightarrow{\mathcal{T}}_{i}\right)\right. \\
& \left.\left.\left.\times\left(\sin 2 \theta \overrightarrow{\mathcal{N}}_{j}-\cos 2 \theta \overrightarrow{\mathcal{T}}_{j}\right)+\left(\sin 2 \theta \overrightarrow{\mathcal{N}}_{i}+\cos 2 \theta \overrightarrow{\mathcal{T}}_{i}\right)\left(\cos 2 \theta \overrightarrow{\mathcal{N}}_{j}+\sin 2 \theta \overrightarrow{\mathcal{T}}_{j}\right)\right]\right\}-(3+\eta)\right],
\end{aligned}
$$

while for the bonds $\langle i j\rangle \perp(a, b)$ it takes the form 


$$
\begin{aligned}
\mathcal{H}_{\perp}= & J \sum_{\langle i j\rangle \perp}\left(\left(1-\frac{1}{2} \eta\right)\left[(1+\cos 2 \theta) \overrightarrow{\mathcal{S}}_{i \mu \mu}+(1-\cos 2 \theta) \overrightarrow{\mathcal{S}}_{i \nu \nu}-\sin 2 \theta \overrightarrow{\mathcal{K}}_{i}\right]\left[(1+\cos 2 \theta) \overrightarrow{\mathcal{S}}_{j \mu \mu}+(1-\cos 2 \theta) \overrightarrow{\mathcal{S}}_{j \nu \nu}-\sin 2 \theta \overrightarrow{\mathcal{K}}_{j}\right]\right. \\
& -\frac{1}{4} \eta\left\{\left[(1-\cos 2 \theta) \overrightarrow{\mathcal{S}}_{i \mu \mu}+(1+\cos 2 \theta) \overrightarrow{\mathcal{S}}_{i \nu \nu}+\sin 2 \theta \overrightarrow{\mathcal{K}}_{i}\right]\left[(1+\cos 2 \theta) \overrightarrow{\mathcal{S}}_{j \mu \mu}+(1-\cos 2 \theta) \overrightarrow{\mathcal{S}}_{j \nu \nu}-\sin 2 \theta \overrightarrow{\mathcal{K}}_{j}\right]\right. \\
& \left.+\left[(1+\cos 2 \theta) \overrightarrow{\mathcal{S}}_{i \mu \mu}+(1-\cos 2 \theta) \overrightarrow{\mathcal{S}}_{i \nu \nu}-\sin 2 \theta \overrightarrow{\mathcal{K}}_{i}\right]\left[(1-\cos 2 \theta) \overrightarrow{\mathcal{S}}_{j \mu \mu}+(1+\cos 2 \theta) \overrightarrow{\mathcal{S}}_{j \nu \nu}+\sin 2 \theta \overrightarrow{\mathcal{K}}_{j}\right]\right\} \\
& \left.+(1+2 \eta)\left(\cos 2 \theta \overrightarrow{\mathcal{N}}_{i}-\sin 2 \theta \overrightarrow{\mathcal{T}}_{i}\right)\left(\cos 2 \theta \overrightarrow{\mathcal{N}}_{j}-\sin 2 \theta \overrightarrow{\mathcal{T}}_{j}\right)-\frac{1}{4}(3+\eta)\right)
\end{aligned}
$$

and the transformed orbital-anisotropy term reads

$$
\mathcal{H}_{\tau}=E_{z} \sum_{i}\left(\cos 2 \theta \overrightarrow{\mathcal{N}}_{i}-\sin 2 \theta \overrightarrow{\mathcal{T}}_{i}\right) .
$$

The transverse excitations were found using the RPA procedure in Eqs. (4.53) and (4.54) which leads to an $(8 \times 8)$ matrix for the eigenenergies. If the operators transformed to $\vec{k}$ space are ordered as $\mathcal{S}_{A \mu \mu}^{+}, \mathcal{S}_{B \mu \mu}^{+}, \mathcal{K}_{A \mu \nu}^{+}, \mathcal{K}_{B \mu \nu}^{+}, \mathcal{S}_{C \mu \mu}^{+}, \mathcal{S}_{D \mu \mu}^{+}, \mathcal{K}_{C \mu \nu}^{+}, \mathcal{K}_{D \mu \nu}^{+}$, one recovers a general structure of the eigenvalue problem,

$$
\left(\begin{array}{cc}
\mathcal{A}-\bar{\omega}_{\vec{k}} \mathcal{I} & \mathcal{B} \\
-\mathcal{B} & -\mathcal{A}-\bar{\omega}_{\vec{k}} \mathcal{I}
\end{array}\right)=0,
$$

where $\mathcal{A}$ and $\mathcal{B}$ are $(4 \times 4)$ symmetric matrices, $\mathcal{I}$ is the (4 $\times 4)$ identity matrix, and $\bar{\omega}_{\vec{k}}=\omega_{\vec{k}} / J$. Using the averages of the diagonal operators in the classical ground state,

$$
\begin{gathered}
\left\langle\mathcal{S}_{A \mu \mu}^{z}\right\rangle=\left\langle\mathcal{S}_{B \mu \mu}^{z}\right\rangle=-\left\langle\mathcal{S}_{C \mu \mu}^{z}\right\rangle=-\left\langle\mathcal{S}_{D \mu \mu}^{z}\right\rangle=\frac{1}{2}, \\
\left\langle\mathcal{N}_{i-}\right\rangle=\frac{1}{2},
\end{gathered}
$$

one finds the following elements of matrix $\mathcal{A}$ :

$$
\begin{aligned}
& \mathcal{A}_{11}= \mathcal{A}_{22}=-\frac{1}{2}\left(1-\frac{1}{2} \eta\right)(1-2 \cos 2 \theta)^{2}+2(2-\eta) \cos ^{4} \theta \\
&+ \frac{1}{2} \eta\left(\frac{3}{2}+\sin ^{2} 2 \theta\right), \\
& \mathcal{A}_{12}=\left[\frac{1}{2}\left(1-\frac{1}{2} \eta\right)(1-2 \cos 2 \theta)^{2}-\eta\left(\frac{3}{4}+\sin ^{2} 2 \theta\right)\right] \gamma_{+}(\vec{k}), \\
& \mathcal{A}_{13}=-\mathcal{A}_{24}=-\frac{1}{2}\left(1-\frac{1}{2} \eta\right) \sin 2 \theta(2-\cos 2 \theta) \\
&-\frac{1}{4}\left(3+\frac{11}{2} \eta\right) \sin 4 \theta-\frac{1}{2} \varepsilon_{z} \sin 2 \theta, \\
& \mathcal{A}_{14}=-\left(1-\frac{1}{2} \eta-2 \cos 2 \theta\right) \sin 2 \theta \gamma_{+}(\vec{k}) \\
& \quad+\frac{\sqrt{3}}{2}[1-(2-\eta) \cos 2 \theta] \gamma_{-}(\vec{k}), \\
& \mathcal{A}_{23}=+\left(1-\frac{1}{2} \eta-2 \cos 2 \theta\right) \sin 2 \theta \gamma_{+}(\vec{k}) \\
& \quad+\frac{\sqrt{3}}{2}[1-(2-\eta) \cos 2 \theta] \gamma_{-}(\vec{k}), \\
& \mathcal{A}_{33}=
\end{aligned}
$$

$$
\mathcal{A}_{34}=\frac{1}{2}(1+2 \cos 4 \theta) \gamma_{+}(\vec{k}),
$$

and the following nonzero elements of matrix $\mathcal{B}$ :

$$
\begin{gathered}
\mathcal{B}_{11}=\mathcal{B}_{22}=\left[\left(1-\frac{1}{2} \eta\right)(1+\cos 2 \theta)-\frac{1}{4} \eta\right](1+\cos 2 \theta) \\
\gamma_{z}(\vec{k}), \\
\mathcal{B}_{33}=\mathcal{B}_{44}=\sin ^{2} 2 \theta \gamma_{z}(\vec{k}), \\
\mathcal{B}_{13}=\mathcal{B}_{31}=-\mathcal{B}_{24}=-\mathcal{B}_{42}=-\left(1-\frac{1}{2} \eta+\cos 2 \theta\right) \sin 2 \theta \gamma_{z}(\vec{k}) .
\end{gathered}
$$

The longitudinal excitations in the A-AF phases were obtained by solving the respective Green function equations for the excitation operators (4.55). After transforming these equations to $\vec{k}$ space, and taking the following sequence of excitation operators: $\mathcal{T}_{A \mu \nu \uparrow}, \mathcal{T}_{B \mu \nu \uparrow}, \mathcal{T}_{A \nu \mu \uparrow}, \mathcal{T}_{B \nu \mu \uparrow}$, $\mathcal{T}_{C \mu \nu \downarrow}, \mathcal{T}_{D \mu \nu \downarrow}, \mathcal{T}_{C \nu \mu \downarrow}, \mathcal{T}_{D \nu \mu \downarrow}$, one finds an eigenvalue problem of the form

$$
\left(\begin{array}{cccc}
\mathcal{P}-\bar{\zeta}_{\vec{k}} \mathcal{I} & \mathcal{R} & +\mathcal{Q} & +\mathcal{Q} \\
-\mathcal{R} & -\mathcal{P}-\bar{\zeta}_{\vec{k}} \mathcal{I} & -\mathcal{Q} & -\mathcal{Q} \\
-\mathcal{Q} & -\mathcal{Q} & -\mathcal{P}-\bar{\zeta}_{\vec{k}} \mathcal{I} & -\mathcal{R} \\
+\mathcal{Q} & +\mathcal{Q} & \mathcal{R} & \mathcal{P}-\bar{\zeta}_{\vec{k}} \mathcal{I}
\end{array}\right)=0
$$

where $\mathcal{P}, \mathcal{R}$, and $\mathcal{Q}$ are symmetric $(2 \times 2)$ matrices, and $\bar{\zeta}_{\vec{k}}$ $=\zeta_{\vec{k}} / J$. The nonvanishing elements are defined as follows:

$$
\begin{aligned}
\mathcal{P}_{11}=\mathcal{P}_{22}= & \frac{1}{2}\left(1-\frac{1}{2} \eta\right)[1-2 \cos 4 \theta+2 \cos 2 \theta(2+\cos 2 \theta)] \\
& +\frac{3}{4} \eta \cos 2 \theta-\frac{3}{2}(1+2 \eta) \cos 4 \theta-\varepsilon_{z} \cos 2 \theta,
\end{aligned}
$$

$$
\begin{gathered}
\mathcal{P}_{12}=\mathcal{P}_{21}=\frac{1}{2}(1+\eta)(1-2 \cos 2 \theta) \gamma_{+}(\vec{k}), \\
\mathcal{R}_{12}=\mathcal{R}_{21}=\frac{1}{2}(1+\eta)(1-2 \cos 2 \theta) \gamma_{+}(\vec{k}), \\
\mathcal{Q}_{11}=\mathcal{Q}_{22}=\eta \sin ^{2} 2 \theta \gamma_{z}(\vec{k}) .
\end{gathered}
$$

As in the AF phases, the coupling between the sublattices $A$ and $C$ and between $B$ and $D$, respectively, is proportional to the weak FM component $\eta$. The mechanism of this coupling is explained in Fig. 8.

\section{APPENDIX D: NEUTRON INTENSITIES IN TRANSVERSE EXCITATIONS}

In this appendix we explain the intensities $\chi(\omega)$ in neutron scattering seen in the presence of orbital degrees of freedom. One can start from the general expression for the cross section for pure magnetic scattering, ${ }^{84}$ 


$$
\begin{aligned}
\frac{d^{2} \sigma}{d \Omega d \omega} \propto & \frac{k_{1}}{k_{0}} \sum_{i j} f_{j}^{*}(\vec{q}) f_{i}(\vec{q}) \frac{1}{2 \pi} \int d t e^{-i \omega t} \\
& \times\left\langle\vec{S}_{i \perp}(0) \vec{S}_{j \perp}(t)\right\rangle e^{-i \vec{q}\left(\vec{R}_{i}-\vec{R}_{j}\right)}
\end{aligned}
$$

where $k_{0}$ and $k_{1}$ are the initial and final momenta, while $\vec{q}$ is the momentum transfer. The spin components at site $i$ and $j$ are perpendicular to $\vec{q}$. By integrating over time $t$ one finds that the neutron cross section (D1) is related to the imaginary part of the spin-spin Green function,

$$
\begin{aligned}
\frac{d^{2} \sigma}{d \Omega d \omega} \propto & \frac{k_{1}}{k_{0}} \sum_{i j} f_{j}^{*}(\vec{q}) f_{i}(\vec{q}) e^{-i \vec{q}\left(\vec{R}_{i}-\vec{R}_{j}\right)} \frac{1}{2 \pi} 2 \operatorname{Im} \\
& \times\left\{\sum_{\alpha}\left\langle\left\langle S_{j \perp}^{\alpha} \mid S_{i \perp}^{\alpha}\right\rangle\right\rangle_{-\omega}\right\} \Theta(\omega),
\end{aligned}
$$

where $\Theta(\omega)=1$ for $\omega>0$, and $\Theta(\omega)=0$ for $\omega<0$, and we took the limit of temperature $T \rightarrow 0$. In order to extract the perpendicular component of the spin-spin correlation function from the Green functions, $\left\langle\left\langle S_{j \perp}^{\alpha} \mid S_{i \perp}^{\alpha}\right\rangle\right\rangle_{-\omega}$, we use the identity

$$
S_{i \perp}^{\alpha}=\sum_{\beta} S_{i}^{\beta}\left(\delta_{\alpha \beta}-\frac{q^{\alpha} q^{\beta}}{q^{2}}\right) .
$$

The components of the Green functions in $\vec{q}$ space, $\left\langle\left\langle S_{\vec{q}}^{\alpha} \mid S_{-\vec{q}}^{\beta}\right\rangle\right\rangle_{-\omega}$, are found using the following properties of the transverse spin-spin functions:

$$
\begin{gathered}
\operatorname{Im}\left\langle\left\langle S_{\vec{q}}^{\alpha} \mid S_{-\vec{q}}^{\beta}\right\rangle\right\rangle_{-\omega}=-\operatorname{Im}\left\langle\left\langle S_{\vec{q}}^{\beta} \mid S_{-\vec{q}}^{\alpha}\right\rangle\right\rangle_{\omega}, \\
\left\langle\left\langle S_{\vec{q}}^{+} \mid S_{-\vec{q}}^{+}\right\rangle\right\rangle_{\omega}=\left\langle\left\langle S_{\vec{q}}^{-} \mid S_{-\vec{q}}^{-}\right\rangle\right\rangle_{\omega}=0,
\end{gathered}
$$

and $\left\langle\left\langle S_{\vec{q}}^{\alpha} \mid S_{-\vec{q}}^{z}\right\rangle\right\rangle_{\omega}=0$ for the wave vectors $\vec{q} \neq \vec{Q}$, where $\vec{Q}$ is the nesting vector. One finds that the neutron cross section normalized per one site may be written as follows:

$$
\frac{1}{N} \frac{d^{2} \sigma}{d \Omega d \omega} \propto \frac{1}{8 \pi} \frac{k_{1}}{k_{0}} \frac{1}{N} \sum_{i j} f_{j}^{*}(\vec{q}) f_{i}(\vec{q}) \chi(\vec{q}),
$$

where $\chi(\vec{q})$ is the neutron scattering intensity which includes the geometrical factor which originates from Eq. (D3). It is proportional to a linear combination of the diagonal and offdiagonal elements of the Green function, and one finds for a two-sublattice magnetic structure, as for example in AFxx and AFzz phases,

$$
\begin{aligned}
\chi(\vec{q})= & \left(1+\frac{q_{z}^{2}}{q^{2}}\right) 2 \operatorname{Im}\left[G_{A A}(\vec{q},-\omega)\right. \\
& \left.+G_{B B}(\vec{q},-\omega)+G_{A B}(\vec{q},-\omega)+G_{B A}(\vec{q},-\omega)\right] \Theta(\omega),
\end{aligned}
$$

with the element $G_{A A}(\vec{q},-\omega)$ standing for the transverse Green function, $\left\langle\left\langle S_{A, \vec{q}}^{+} \mid S_{A,-\vec{q}}^{-}\right\rangle\right\rangle_{-\omega}$, etc., and the indices $A$ and $B$ refer to two sublattices. The explicit formula in terms of the spectral intensities $\mathcal{A}_{m n}^{(\nu)}(\vec{q})$ is given by

$$
\begin{aligned}
\chi(\vec{q})= & \left(1+\frac{q_{z}^{2}}{q^{2}}\right) \sum_{\nu(>0)}\left[\mathcal{A}_{A A}^{(\nu)}(\vec{q})+\mathcal{A}_{B B}^{(\nu)}(\vec{q})+\mathcal{A}_{A B}^{(\nu)}(\vec{q})\right. \\
& \left.+\mathcal{A}_{B A}^{(\nu)}(\vec{q})\right] \delta\left(\omega-\omega_{\vec{q}}^{(\nu)}\right) .
\end{aligned}
$$

We have used Eq. (D7) to determine the contributions to the neutron cross section due to different excitations, as analyzed in Sec. IV and presented in Figs. 9-12. The generalization to the case of four-sublattice structures found in the MOFFA and MOAFF phases is straightforward.
${ }^{1}$ For a review, see M. Imada, A. Fujimori, and Y. Tokura, Rev. Mod. Phys. 70, 1039 (1998).

${ }^{2}$ M. Kollar, R. Strack, and D. Vollhardt, Phys. Rev. B 53, 9225 (1996); D. Vollhardt, M. Blümmer, K. Held, J. Schlipf, and M. Ulmke, Z. Phys. B 103, 283 (1997); T. Hanisch, G.S. Uhrig, and E. Müller-Hartmann, Phys. Rev. B 56, 13960 (1997).

${ }^{3}$ E. Dagotto, Rev. Mod. Phys. 66, 763 (1994).

${ }^{4}$ H. Tsunetsugu, M. Sigrist, and K. Ueda, Rev. Mod. Phys. 69, 809 (1997).

${ }^{5}$ K.I. Kugel and D.I. Khomskii, Zh. Éksp. Teor. Fiz 64, 1429 (1973) [Sov. Phys. JETP 37, 725 (1973)].

${ }^{6}$ L.F. Feiner, A.M. Oleś, and J. Zaanen, Phys. Rev. Lett. 78, 2799 (1997)

${ }^{7}$ D.I. Khomskii and G.A. Sawatzky, Solid State Commun. 102, 87 (1997).

${ }^{8}$ J. Zaanen, A. M. Oleś, and L. F. Feiner, in Dynamics of Magnetic Fluctuations in High Temperature Superconductors, edited by G. Reiter, P. Horsch, and G. C. Psaltakis (Plenum Press, New York, 1991), p. 241; L.F. Feiner, A.M. Oleś, and J. Zaanen, J. Magn. Magn. Mater. 140-144, 1941 (1995).
${ }^{9}$ K.I. Kugel and D.I. Khomskii, Usp. Fiz. Nauk 136, 621 (1982) [Sov. Phys. Usp. 25, 231 (1982)].

${ }^{10}$ A.I. Liechtenstein, V.I. Anisimov, and J. Zaanen, Phys. Rev. B 52, R5467 (1995).

${ }^{11}$ V.I. Anisimov, I.S. Elfimov, M.A. Korotin, and K. Terakura, Phys. Rev. B 55, 15494 (1997).

${ }^{12}$ P. Fulde and I. Peschel, Adv. Phys. 21, 1 (1972); Y.Y. Hsieh and M. Blume, Phys. Rev. B 6, 2684 (1972).

${ }^{13}$ D.L. Cox, Phys. Rev. Lett. 59, 1240 (1987).

${ }^{14}$ T. Mizokawa and A. Fujimori, Phys. Rev. B 51, 12880 (1995); 54, 5368 (1996).

${ }^{15}$ S. Ishihara, J. Inoue, and S. Maekawa, Physica C 263, 130 (1996); Phys. Rev. B 55, 8280 (1997).

${ }^{16}$ R. Shiina, T. Nishitani, and H. Shiba, J. Phys. Soc. Jpn. 66, 3159 (1997).

${ }^{17}$ L.F. Feiner and A.M. Oleś, Phys. Rev. B 59, 3295 (1999).

${ }^{18}$ J. Zaanen and A.M. Oleś, Phys. Rev. B 48, 7197 (1993).

${ }^{19}$ J. Zaanen, A.M. Oleś, and P. Horsch, Phys. Rev. B 46, 5798 (1992).

${ }^{20}$ E. Dagotto, J. Riera, A. Sandvik, and A. Moreo, Phys. Rev. Lett. 76, 1731 (1996). 
${ }^{21}$ E. Müller-Hartmann and E. Dagotto, Phys. Rev. B 54, R6819 (1996).

${ }^{22}$ Y. Okimoto, T. Katsufuji, T. Ishikawa, T. Arima, and Y. Tokura, Phys. Rev. B 55, 4206 (1997).

${ }^{23}$ A.J. Millis, B.I. Shraiman, and R. Mueller, Phys. Rev. Lett. 77, 175 (1997).

${ }^{24}$ A. Takahashi and H. Shiba, Eur. Phys. J. B 5, 413 (1998).

${ }^{25}$ J. van den Brink and D. Khomskii, Phys. Rev. Lett. 82, 1016 (1999).

${ }^{26}$ W. Weber, Z. Phys. B 70, 323 (1988).

${ }^{27}$ D.I. Khomskii, Physica B 171, 44 (1991).

${ }^{28}$ J.B. Grant and A.K. McMahan, Phys. Rev. B 46, 8440 (1992).

${ }^{29}$ J.H. Jefferson, H. Eskes, and L.F. Feiner, Phys. Rev. B 45, 7959 (1992); L.F. Feiner, J.H. Jefferson, and R. Raimondi, ibid. 53, 8751 (1996); R. Raimondi, L.F. Feiner, and J.H. Jefferson, ibid. 53, 8774 (1996).

${ }^{30}$ M. Cyrot and C. Lyon-Caen, J. Phys. (Paris) 36, 253 (1975); S. Inagaki, J. Phys. Soc. Jpn. 39, 596 (1975).

${ }^{31}$ J. Spałek and K.A. Chao, J. Phys. C 13, 5241 (1980).

${ }^{32} \mathrm{~J}$. B. Goodenough, Magnetism and Chemical Bond (Interscience, New York, 1963).

${ }^{33}$ D.I. Khomskii and K.I. Kugel, Solid State Commun. 13, 763 (1974).

${ }^{34}$ C. Castellani C.R. Natoli and J. Ranninger, Phys. Rev. B 18, 4945 (1978); 18, 4967 (1978); 18, 5001 (1978); T. M. Rice, in Spectroscopy of Mott Insulators and Correlated Metals, edited by A. Fujimori and Y. Tokura (Springer-Verlag, Berlin, 1995).

${ }^{35}$ W. Bao, C. Broholm, G. Aeppli, P. Dai, J.M. Honig, and P. Metcalf, Phys. Rev. Lett. 78, 507 (1997); W. Bao, C. Broholm, G. Aeppli, S.A. Carter, P. Dai, T.F. Rosenbaum, J.M. Honig, P. Metcalf, and S.F. Trevino, Phys. Rev. B 58, 12727 (1998).

${ }^{36}$ S.Y. Ezhov, V.I. Anisimov, D.I. Khomskii, and G.A. Sawatzky, Phys. Rev. Lett. 83, 4136 (1999).

${ }^{37}$ S. Ishihara, M. Yamanaka, and N. Nagaosa, Phys. Rev. B 56, 686 (1997).

${ }^{38}$ H. Bethe, Z. Phys. 64, 188 (1931).

${ }^{39}$ S.R. White, R.M. Noack, and D.J. Scalapino, Phys. Rev. Lett. 73, 886 (1994).

${ }^{40}$ E. Dagotto and T.M. Rice, Science 271, 618 (1996); M. Troyer, H. Tsunetsugu, and T.M. Rice, Phys. Rev. B 53, 251 (1996); B. Normand and T.M. Rice, ibid. 54, 7180 (1996).

${ }^{41}$ P. Chandra and B. Doucot, Phys. Rev. B 38, 9335 (1988); A. Moreo, E. Dagotto, T. Jolicoeur, and J. Riera, ibid. 42, 6283 (1990); N. Read and S. Sachdev, Phys. Rev. Lett. 66, 1773 (1991); A.V. Dotsenko and O.P. Sushkov, Phys. Rev. B 50, 13821 (1994); N.B. Ivanov, S.E. Krüger, and J. Richter, ibid. 53, 2633 (1996).

${ }^{42}$ A. Chubukov, Phys. Rev. B 44, 392 (1991).

${ }^{43}$ A.J. Millis and H. Monien, Phys. Rev. B 50, 16606 (1994); A.W. Sandvik and D.J. Scalapino, Phys. Rev. Lett. 72, 2777 (1994).

${ }^{44}$ A.V. Chubukov and D.K. Morr, Phys. Rev. B 52, 3521 (1995).

${ }^{45}$ S. Taniguchi, T. Nishikawa, Y. Yasui, Y. Kobayashi, M. Sato, T. Nishioka, M. Kontani, and K. Sano, J. Phys. Soc. Jpn. 64, 2758 (1995)

${ }^{46}$ K. Ueda, H. Kontani, M. Sigrist, and P.A. Lee, Phys. Rev. Lett. 76, 1932 (1996); M. Troyer, H. Kontani, and K. Ueda, ibid. 76, 3822 (1996); O.A. Starykh, M.E. Zhitomirsky, D.I. Khomskii, R. Singh, and K. Ueda, ibid. 77, 2558 (1996); S.R. White, ibid. 77, 3633 (1996).

${ }^{47}$ J. Zaanen and A.M. Oleś, Phys. Rev. B 37, 9423 (1988).

${ }^{48}$ The covalency due to $p-d$ hybridization is responsible for the admixture of the $d^{10} L$ configuration and leads to some quantitative changes of the parameters in the present effective model which describes only $d$ orbitals at transition-metal ions.

${ }^{49}$ J.C. Slater and G.F. Koster, Phys. Rev. 94, 1498 (1954).

${ }^{50}$ For convenience, we defined $U$ as the average excitation energy of the $d^{8}$ configuration. Therefore $U$ is not the interorbital Coulomb element, and with the present definition of $J_{H}$ as equal to the singlet-triplet splitting, the interorbital interaction between holes of opposite (equal) spins is $U-J_{H} / 2\left(U-J_{H}\right)$.

${ }^{51}$ J. S. Griffith, The Theory of Transition Metal Ions (Cambridge University Press, Cambridge, 1971).

${ }^{52}$ A.M. Oleś, Phys. Rev. B 28, 327 (1983).

${ }^{53}$ M.S. Hybertsen, M. Schlüter, and N.E. Christensen, Phys. Rev. B 39, 9028 (1989).

${ }^{54}$ J. Zaanen and G.A. Sawatzky, J. Solid State Chem. 88, 8 (1990).

${ }^{55}$ H. Eskes and J.H. Jefferson, Phys. Rev. B 48, 9788 (1993); T. Tohyama and S. Maekawa, ibid. 49, 3596 (1994).

${ }^{56}$ B. Keimer, N. Belk, R.J. Birgeneau, A. Cassanho, C.Y. Chen, M. Greven, M.A. Kastner, A. Aharony, Y. Endoh, R.W. Erwin, and G. Shirane, Phys. Rev. B 46, 14034 (1992).

${ }^{57}$ K.A. Chao, J. Spałek, and A.M. Oleś, J. Phys. C 10, L271 (1977); Phys. Rev. B 18, 3453 (1978).

${ }^{58}$ The Hamiltonian given in Ref. 18 does not contain the pair hopping terms (see Ref. 52) in Eq. (2.3), and gives thus a different multiplet structure from that obtained in Eqs. (2.5).

${ }^{59} \mathrm{~A}$ simplified multiplet structure used in Refs. 5 and 9 leads to a different expression. We also note that this earlier proposed form does not observe the cubic symmetry in the orbital space.

${ }^{60}$ B. Frischmuth, F. Mila, and M. Troyer, Phys. Rev. Lett. 82, 835 (1999).

${ }^{61}$ A.P. Kampf, Phys. Rep. 249, 219 (1994).

${ }^{62}$ J. Kanamori, J. Appl. Phys. 31, 14S (1960).

${ }^{63}$ M.A. Kastner, R.J. Birgeneau, G. Shirane, and Y. Endoh, Rev. Mod. Phys. 70, 897 (1998).

${ }^{64}$ Y. Ito and J. Akimutsu, J. Phys. Soc. Jpn. 33, 1333 (1976); L.D. Khoi and P. Veillet, Phys. Rev. B 11, 4128 (1975); W. Kleemann and Y. Farge, J. Phys. (Paris) 36, 1293 (1975).

${ }^{65}$ L.F. Feiner, A.M. Oleś, and J. Zaanen, J. Phys.: Condens. Matter 10, L555 (1998).

${ }^{66}$ J. van den Brink, W. Stekelenburg, D.I. Khomskii, G.A. Sawatzky, and K.I. Kugel, Phys. Rev. B 58, 10276 (1998).

${ }^{67}$ B. Sutherland, Phys. Rev. B 12, 3795 (1975).

${ }^{68}$ M. Takahashi, Phys. Rev. B 40, 2494 (1989).

${ }^{69}$ A. Auerbach, Interacting Electrons and Quantum Magnetism (Springer, New York, 1994).

${ }^{70}$ D.N. Zubarev, Usp. Fiz. Nauk 71, 71 (1960) [Sov. Phys. Usp. 3, $320(1960)]$.

${ }^{71}$ S.B. Haley and P. Erdös, Phys. Rev. B 5, 1106 (1972).

${ }^{72}$ G. Khaliullin and V. Oudovenko, Phys. Rev. B 56, R14 243 (1997).

${ }^{73}$ F. Mila, B. Frischmuth, A. Deppeler, and M. Troyer, Phys. Rev. Lett. 82, 3697 (1999).

${ }^{74}$ D.H. Tennant, T.G. Perring, R.A. Cowley, and S.E. Nagler, Phys. Rev. Lett. 70, 4003 (1993); H. Tennant, R.A. Cowley, S.E. Nagler, and A.M. Tsvelik, Phys. Rev. B 52, 13368 (1995); D.H. Tennant, S.E. Nagler, D. Welz, G. Shirane, and K. Yamada, ibid. 52, 13381 (1995).

${ }^{75}$ K. Hirota, N. Kaneko, A. Nishizawa, and Y. Endoh, J. Phys. Soc. Jpn. 65, 3736 (1996); F. Moussa, H. Henion, J. RodriguezCarvajal, H. Moudden, L. Pinsard, and A. Revcolevschi, Phys. Rev. B 54, 15149 (1996). 
${ }^{76}$ L. F. Feiner, A. M. Oleś, and J. Zaanen (unpublished).

${ }^{77}$ The expansion (5.3) is exact and holds for the global spin operator $S_{i}^{z}$, while a similar relation holds for the spin operators projected on one orbital state, being in the AFxx phase $S_{i x x}^{z}=\frac{1}{2} \rrbracket$ $-S_{i x x}^{-} S_{i x x}^{+}-\frac{1}{2} K_{i z x}^{-} K_{i x z}^{+}-\frac{1}{2} T_{i z x \uparrow}^{-} T_{i x z \uparrow}^{+}$, if $i \in A$. Equation (5.3) was also used in Ref. 65 to calculate the quantum correction to the order parameter $\delta\left\langle S^{z}\right\rangle$ in the AFxx and AFzz phase at $J_{H}$ $=0$. The pertinent equation is Eq. (15), with the coefficient in front of $\left\langle S_{i}^{-} \sigma_{i}^{-} S_{i}^{+} \sigma_{i}^{+}\right\rangle$being $\frac{1}{4}$. This coefficient follows from a relation between the present operators $K_{i \alpha \beta}^{ \pm}$and the composite spin-and-orbital operators, $K_{i}^{ \pm}=\frac{1}{2} S_{i}^{ \pm} \sigma_{i}^{ \pm}$, and was misprinted in Eq. (15) of Ref. 65.

${ }^{78}$ In the present model the modes become soft along particular lines in the BZ only at $\eta=0$, while at finite $\eta$ the soft-mode behavior is limited to the high-symmetry points. This is due to making an expansion in $\eta$ on the spin-orbital model (2.9), yielding Eq. (2.14) which is only accurate up to first order in $\eta$, while also higher-order terms enter the calculation of the transverse spectra. Therefore the energies of the modes remain finite (but very shallow $\propto \eta^{2}$ ) close to the high-symmetry lines where they would become soft, if higher-order terms were also included in a consistent way.

${ }^{79}$ Our presentation of this argument was not accurate in the previous paper (Ref. 6) as the expansion around the softening lines gives only an analytic result with a logarithmic divergence for the AFxx phase.

${ }^{80}$ Y. Kitaoka, T. Kobayashi, A. Koda, H. Wakabayashi, Y. Niino, H. Yamakage, S. Taguchi, K. Amaya, K. Yamaura, M. Takano, A. Hirano, and R. Kanno, J. Phys. Soc. Jpn. 67, 3703 (1998).

${ }^{81}$ K. Hirakawa, H. Kadowaki, and K. Ubokoshi, J. Phys. Soc. Jpn. 54, 3526 (1985); K. Yamaura, M. Takano, A. Hirano, and R. Kanno, J. Solid State Chem. 127, 109 (1997).

${ }^{82}$ P. F. Bongers, Ph.D. thesis, University of Leiden, 1957.

${ }^{83}$ Y.Q. Li, M. Ma, D.N. Shi, and F.C. Zhang, Phys. Rev. Lett. 81, 3527 (1998).

${ }^{84}$ P. G. de Gennes, Theory of Neutron Scattering by Magnetic Crystals, in Magnetism, Vol. IV, edited by G. T. Rado and H. Suhl (Academic Press, New York, 1963), p. 115. 